\title{
Single-cell profiling of immune system alterations in lymphoid, barrier and solid tissues in aged mice
}

\author{
Sinduya Krishnarajah ${ }^{1}$, Florian Ingelfinger ${ }^{1,2}$, Ekaterina Friebel ${ }^{1,2}$, Dilay Cansever ${ }^{1}$, Ana Amorim¹, \\ Myrto Andreadou', David Bamert', Gioana Litscher', Mirjam Lutz', Maud Mayoux', Sarah Mundt', \\ Frederike Ridder', Colin Sparano', Sebastian Anton Stifter ${ }^{1}{ }^{1}$, Can Ulutekin', Susanne Unger', \\ Marijne Vermeer', Pascale Zwicky', Melanie Greter ${ }^{1}{ }^{1}$, Sonia Tugues', Donatella De Feo ${ }^{1,3}$ and \\ Burkhard Becher (1,3凶
}

\begin{abstract}
Aging exerts profound and paradoxical effects on the immune system, at once impairing proliferation, cytotoxicity and phagocytosis, and inducing chronic inflammation. Previous studies have focused on individual tissues or cell types, while a comprehensive multisystem study of tissue-resident and circulating immune populations during aging is lacking. Here we reveal an atlas of age-related changes in the abundance and phenotype of immune cell populations across 12 mouse tissues. Using cytometry-based high parametric analysis of 37 mass-cytometry and 55 spectral flow-cytometry parameters, mapping samples from young and aged animals revealed conserved and tissue-type-specific patterns of both immune atrophy and expansion. We uncovered clear phenotypic changes in both lymphoid and myeloid lineages in aged mice, and in particular a contraction in natural killer cells and plasmacytoid dendritic cells. These changes correlated with a skewing towards myelopoiesis at the expense of early lymphocyte genesis in aged mice. Taken together, this atlas represents a comprehensive, systematic and thorough resource of the age-dependent alterations of the mammalian immune system in lymphoid, barrier and solid tissues.
\end{abstract}

T he continuously increasing life expectancy of the global population is associated with a growing number of morbid years in older adults. This aged population is more prone to infections and cancer, and alterations to the aging immune system are held to be the underlying mechanism. Age-related changes within the immune system can be broken down into two main processes: first, a gradual decline in the function of all major cellular leukocyte subsets, termed 'immunosenescence" ${ }^{1}$; and second, the emergence of chronic low-level inflammation-so-called inflammaging ${ }^{2}$-across various tissues ${ }^{3,4}$. Together, these age-related processes are thought to be risk factors for infectious diseases, increased mortality from seasonal viruses ${ }^{5}$, cancer ${ }^{6}$, cardiovascular diseases ${ }^{7}$ and chronic inflammatory disorders 8 . In addition, due to impaired immunological memory in the older adults, low response to vaccination has become a substantial population-wide issue ${ }^{9}$. Consequently, it is important to decipher the changes to the immune system with age to understand the role of such alterations in increased morbidity, and how they might be ameliorated in the future.

Previous research has primarily focused on describing the agerelated changes to adaptive immunity ${ }^{10}$ and the consequences of aging lymphocytes on mounting an immune response to bacterial ${ }^{11}$ or viral ${ }^{12}$ infections. Several studies have characterized the populations of lymphoid cells in the blood of aging humans ${ }^{13,14}$, but it is unknown how circulating levels of immune cell populations compare to those in tissues, which are profoundly important for overall health. Mouse studies have documented a decreased number of classic $T$ cells during aging ${ }^{15,16}$, but again a comprehensive assessment of lymphoid populations and their relative abundance across various tissues is missing. Similarly for myeloid cells, reports of the phenotypic and functional changes across monocytes, macrophages and polymorphonucleated phagocytes have been contradictory ${ }^{17}$ or are focused on an exemplary tissue or $\operatorname{organ}^{4,18}$. There is thus the clear need to map systematically the age-related changes within different myeloid subsets, particularly under physiological conditions and across various tissues. Phagocytes are highly auto-fluorescent, a feature that greatly interferes with classic polychromatic-based cytometry analysis. Furthermore, because numerous canonical myeloid cell markers are shared across most lineages, to identify all myeloid lineages reliably within a range of organs, an exhaustive combination of cell surface markers is required.

Here we present a comprehensive single-cell immunophenotyping atlas of the immune cell populations in 12 different tissues from young and aged C57/BL6 inbred mice. To overcome the technical issues described above, we used mass cytometry, also termed cytometry by time-of-flight (CyTOF), to characterize the myeloid cell subpopulations using a single comprehensive panel and found both tissue-specific and conserved cross-tissue differences between young adult and aged mice. We subsequently went on to confirm our findings with two validation cohorts and a different, independent technique-namely spectral flow cytometry-to establish the robustness of our initial conclusions (two independent cohorts of $n=10$ young and $n=10$ aged mice) and further interrogate the lymphoid and hematopoietic stem cell (HSC) compartments with high resolution. By comparing tissues classified as internal organs, barrier and lymphoid tissues, we observed patterns of age-related alterations across tissue-resident innate immune cell populations, with implications for the capacity of tissues to regenerate and combat pathogenic threats. These data (https://zenodo.org/deposit/5593273) provide a valuable resource into the changes of leukocyte composition, phenotype and function in mammalian tissues during aging. 


\section{Results}

Generating an immune atlas from young and aged mouse tissues. First, we sought to design a mass-cytometry panel and experimental protocol to identify all major innate myeloid lineages across 12 tissues: lung, liver, visceral adipose tissue (VAT), brown adipose tissue (BAT), small intestine, thymus, testes, mesenteric lymph nodes (MesLNs), skin-draining lymph nodes (sdLNs), spleen, blood and bone marrow (BM). For our initial analysis cohort, we devised four experimental groups $(n=4$ young adult male, $n=4$ young adult female; $n=4$ aged female; and $n=3$ aged male C57/BL6 mice) to allow immune comparisons between age as well as sex. We devised similar group sizes for our subsequent validation cohorts, as described in Extended Data Fig. 1 and the Methods section.

Male and female mice aged around 7-8 weeks ('young'), 16 weeks ('middle') or 18-20 months ('aged') were acquired from the same vendor. Our mice showed no physical signs of illness or macroscopic signs of cancerous growths at the time of analysis. Both our initial and validation cohorts of mice have been housed under identical conditions from birth until purchase by the vendor. This was done to avoid facility-specific and induced differences in the readouts, such as alterations to the microbiome of the mice. Furthermore, we aimed to increase the standards of our study's reproducibility by acquiring a mouse cohort which would be available to other researchers for purchase and confirmation experiments.

We first analyzed the cells of the immune compartment using mass cytometry (Fig. 1a). Using unsupervised representation learning techniques and meta-clustering based on self-organizing maps $^{19,20}$, we identified all canonical immune lineages in tissues from both young and old mice (Fig. 1a).

To gather an overview of the immune populations present in our samples, mass-cytometry data were visualized using uniform manifold approximation and projection (UMAP)-dimensionality reduction $^{21}$ on the total concatenated leukocyte fraction from all mice and within each tissue group. The total number of cells depicted per UMAP visualization was normalized per experimental group. Cells were then further grouped using FlowSOM, a self-organizing map that clusters cells (here depicted in different colors) depending on their overall similarities in marker expression ${ }^{19}$. All live/CD45 ${ }^{+}$or myeloid cells per sample were included for the FlowSOM analysis in order better to train the algorithm and improve cluster identification. We identified the major $\mathrm{T}$ cell lineages (CD3 ${ }^{\text {pos }} \mathrm{CD} 90^{\text {pos }}$ cells), further identified by $\mathrm{CD}^{\text {pos }}{ }^{\text {(T }} \mathrm{T}$ helper cells), $\mathrm{CD} 8^{\text {pos }}$ (cytotoxic $\mathrm{T}$ cells), or non- $\alpha \beta \mathrm{T}$ cells $\left(\mathrm{CD} 3^{\text {pos }} \mathrm{CD} 4^{\text {low }} \mathrm{CD} 8^{\text {low }}\right.$ cells such as $\gamma \delta \mathrm{T}$ cells among other unconventional T cell subsets), B cells (B220 ${ }^{\text {high }} \mathrm{MHCII}{ }^{\text {high }}$ Siglec$\mathrm{H}^{\text {neg }}$ ), natural killer (NK) cells (NK1.1 $1^{\text {high }}$ ) and Innate Lymphoid Cells (ILCs) (CD90 pos $\left.\mathrm{CD} 3^{\text {neg }} \mathrm{NK} 1.1^{\text {low }}\right)$ (Fig. 1b).

As lymphoid cells are many times more abundant in most tissues compared to myeloid cells, we then gated out the lymphocytes subsets to enable the identification of all myeloid lineages, including smaller populations such as tissue-resident dendritic cell (DC) subsets. We further quantified the phagocytic populations within all the analyzed organs as a percentage of the total myeloid compartment, as opposed to the total CD $45^{\text {pos }}$ fraction. As visualized in the representative heatmap (Fig. 1c), using additional markers ${ }^{22-24}$, we were able to discern the commonly present myeloid popula- tions across most tissues. In the majority of tissues we defined classic monocytes (Ly6C ${ }^{\text {high }} \mathrm{CCR} 2^{\text {high }}$ ); non-classic monocytes (expressing Ly6C ${ }^{\text {int/low }} \mathrm{CX} 3 \mathrm{CR} 1^{\text {high }} \mathrm{PD}-\mathrm{L} 1^{\text {high }}$ in combination with CD88 ${ }^{\text {high }}$ 4 $/ 80^{\text {high }}$ ); monocyte-derived cells ( Ly6C $\left.^{\text {high }} \mathrm{MHCII}^{\text {high }}\right)$; DC subsets $\left(\mathrm{CD} 11 \mathrm{c}^{\text {pos }} \mathrm{MHCII}{ }^{\text {pos }}\right.$ cells, with XCR $1{ }^{\text {high }} \mathrm{CD} 24^{\text {high }}$ representing conventional dendritic cells 1 ( $\mathrm{cDC} 1 \mathrm{~s})$, and $\mathrm{CD} 11 \mathrm{~b}^{\text {high }} \mathrm{CD} 172 \mathrm{a}^{\text {high }}$ cDC2s, B220 pos Siglec- $\mathrm{H}^{\text {pos }}$ plasmacytoid dendritic cells ( $\mathrm{pDCs}$ )); various tissue macrophage subsets (expressing CD11b with a combination of markers such as F4/80 $0^{\text {high }}$, MerTK ${ }^{\text {high }}$, CD $64^{\text {high }}$, among other tissue-specific markers); as well as granulocytes such as neutrophils (Ly6G $\mathrm{G}^{\mathrm{pos}}$ ); eosinophils (Siglec-F $\mathrm{F}^{\mathrm{pos}}$ ); and mast cells (FceR1a ${ }^{\mathrm{pos}}$ ) (Fig. 1c). While it has recently been shown that the majority of pDCs arise from lymphoid progeny ${ }^{25}$, we have here analyzed these cells alongside their myeloid counterparts due to their main tissue functions (namely antigen expression) matching their myeloid kin; their effector role in bridging the two arms of the immune response; and to increase the resolution on this small population within tissues. By identifying all major myeloid and lymphoid subsets using a single comprehensive panel applicable to all tissues, we demonstrated the robustness and specificity of the mass-cytometry panel designed in this study.

To create a comprehensive immune-phenotyping map as a resource to compare cellular changes between young and aged mice, we aimed to measure age-related alterations in the relative abundance of tissue leukocytes across representative lymphoid and nonlymphoid murine tissues. In addition, we assessed the frequency changes and their relative abundance between sexes in aging. A summary of those features is available for download (https://zenodo.org/ deposit/5593273). Furthermore, while the immunophenotyping has been carried out for the full list of 12 organs listed previously, to depict the scope of our aging immune atlas, we here go on to present exemplary murine tissues with noteworthy age-related changes in cellular composition and phenotypic variances, while introducing our analysis pipeline as well as the different classes of murine tissues we have analyzed. To highlight the general agreement between male and female age-related leukocyte frequency changes, we applied multidimensional scaling to the total leukocytes, in which the distances, or relative similarities, between experimental groups were calculated by pooling the frequencies of all leukocyte populations as per age and sex group ${ }^{26}$. We then visualized the mean antigen expressions across leukocytes using a heatmap with unsupervised hierarchical clustering ${ }^{27}$ (Extended Data Fig. 2) and found that peripheral tissues have very few sex dimorphisms. However, in the BM we observed a neutrophil subsets-driven sex dimorphism particularly in young mice (Extended Data Fig. 2c) ${ }^{28}$. In this case, we present the lung, sdLN and VAT as representative barrier, lymphoid and specialized connective tissues.

Aged lung exhibits pan-reduction of tissue-resident cells. The lung represents one of the best studied murine tissues with regards to the myeloid compartment ${ }^{29}$, both in development ${ }^{30}$ and aging ${ }^{31}$. Thus, the lung represents a canonical barrier tissue affected by agerelated functional decline.

Our initial characterization of the lung lymphoid populations (Fig. 2a,b) showed a significant $(P=0.006)$ mean reduction in $\mathrm{T}$

Fig. 1 | Mass-cytometry and spectral flow-cytometry-based detection of immune cells allows identification of major leukocyte subsets in young and aged mice. a, Schematic diagram of experimental workflow. Briefly, two cohorts of young adult and aged mice of both sexes were culled using an overdose of pentobarbital. Organs were harvested, and the tissues were digested to extract tissue-resident leukocytes. Following antibody labeling, marker expression data were acquired using a mass cytometer or spectral flow cytometer. The data were further analyzed using high-dimensional analysis tools incorporating machine-learning algorithms. b. Modified scaffold map of the total CD45 pos compartment of exemplary mass-cytometry data (cells pooled from $n=1$ liver, $n=1$ lung and $n=1$ spleen). Corresponding heatmap depicts median marker expression within each FlowSOM-derived cluster. $\mathbf{c}$, Modified scaffold map of the total myeloid compartment of exemplary mass-cytometry data, after gating out the lymphoid cells presented in $\mathbf{b}$ (cells pooled from $n=1$ liver, $n=1$ lung and $n=1$ spleen). Corresponding heatmap depicts median marker expression of FlowSOM-derived clusters. Also see details in Extended Data Fig. 1. 
helper cell frequency in lungs from aged mice (approximately $50 \%$ decline relative to lungs from young mice). This age-driven phenotypic change has been reported previously ${ }^{32}$. Similarly, we noted a significant drop in NK cell frequency with age (more than halving from an average of $16.5 \%$ in the young adult group to $7.1 \%$ in the aged mice, $P=0.006$ ) (Fig. $2 \mathrm{~b}$ ). In turn, unconventional T cells

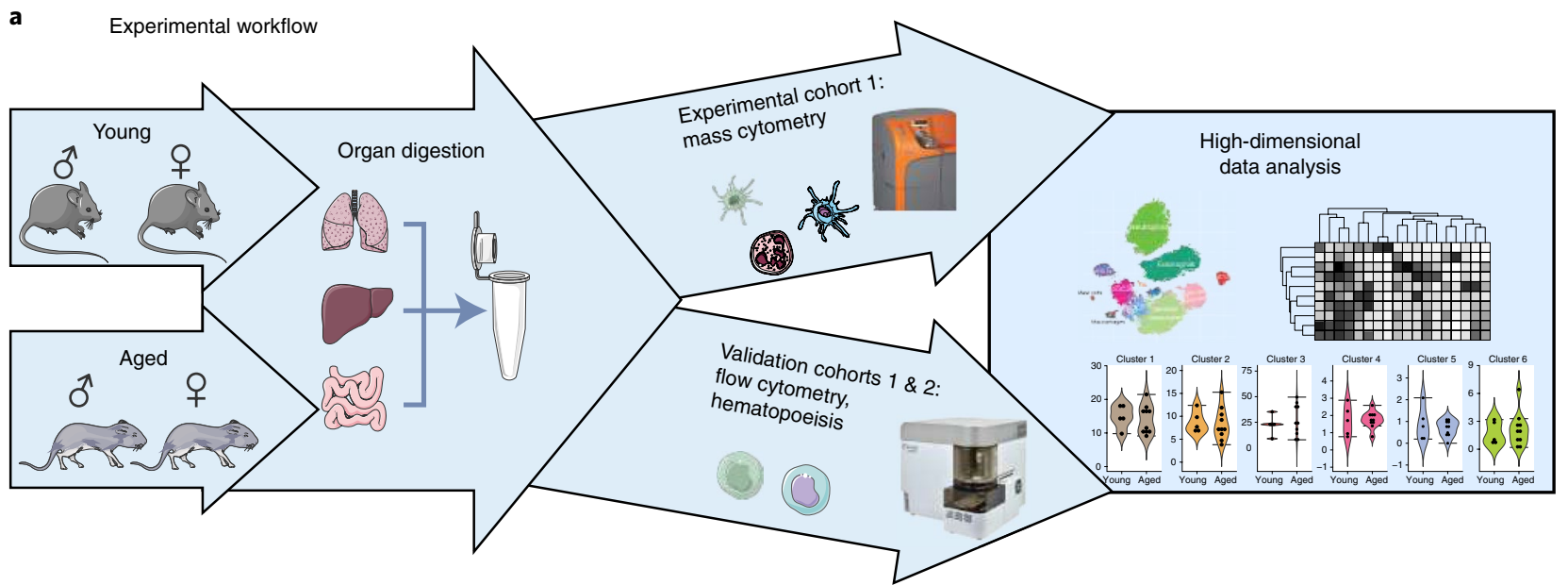

b

Expression profile of lymphoid subsets: representative organs liver; lung and spleen pooled
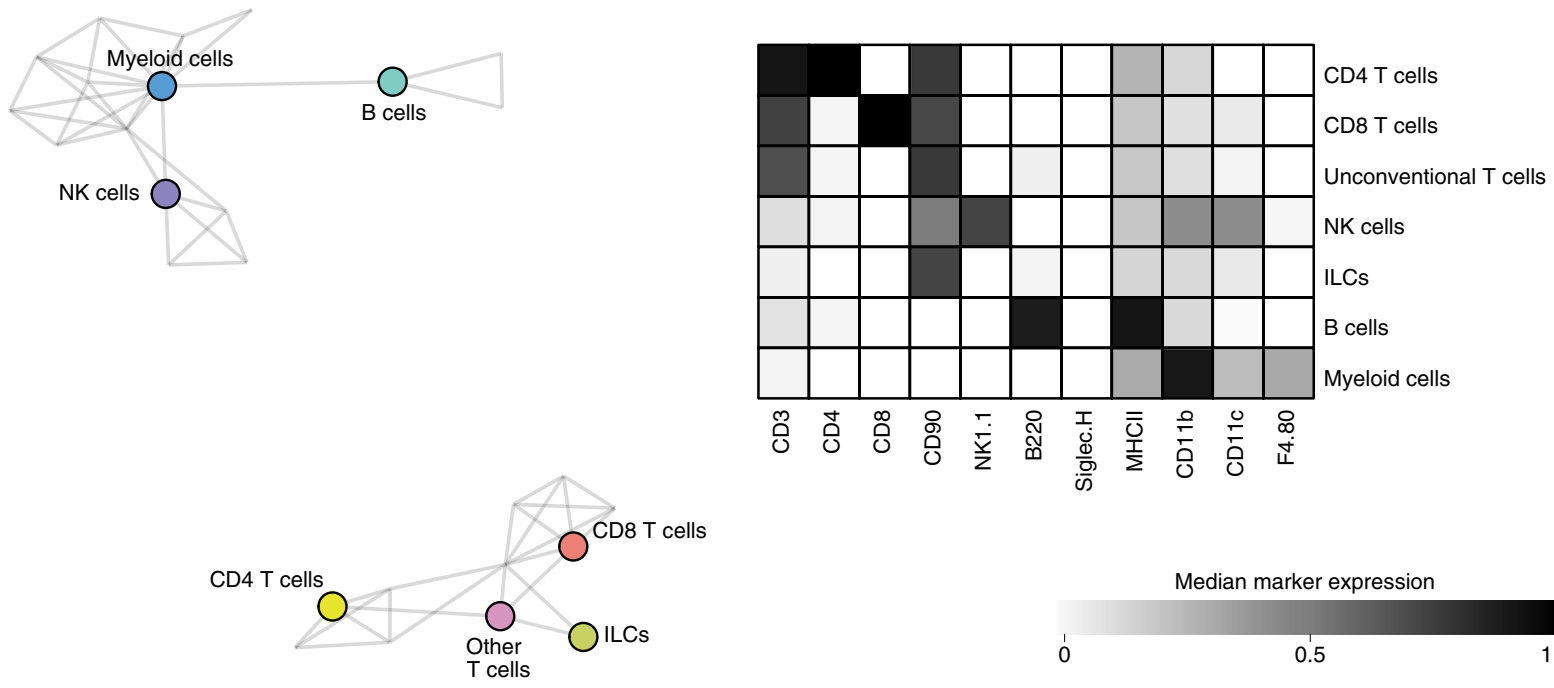

c Expression profile of myeloid subsets: representative organs liver; lung and spleen pooled
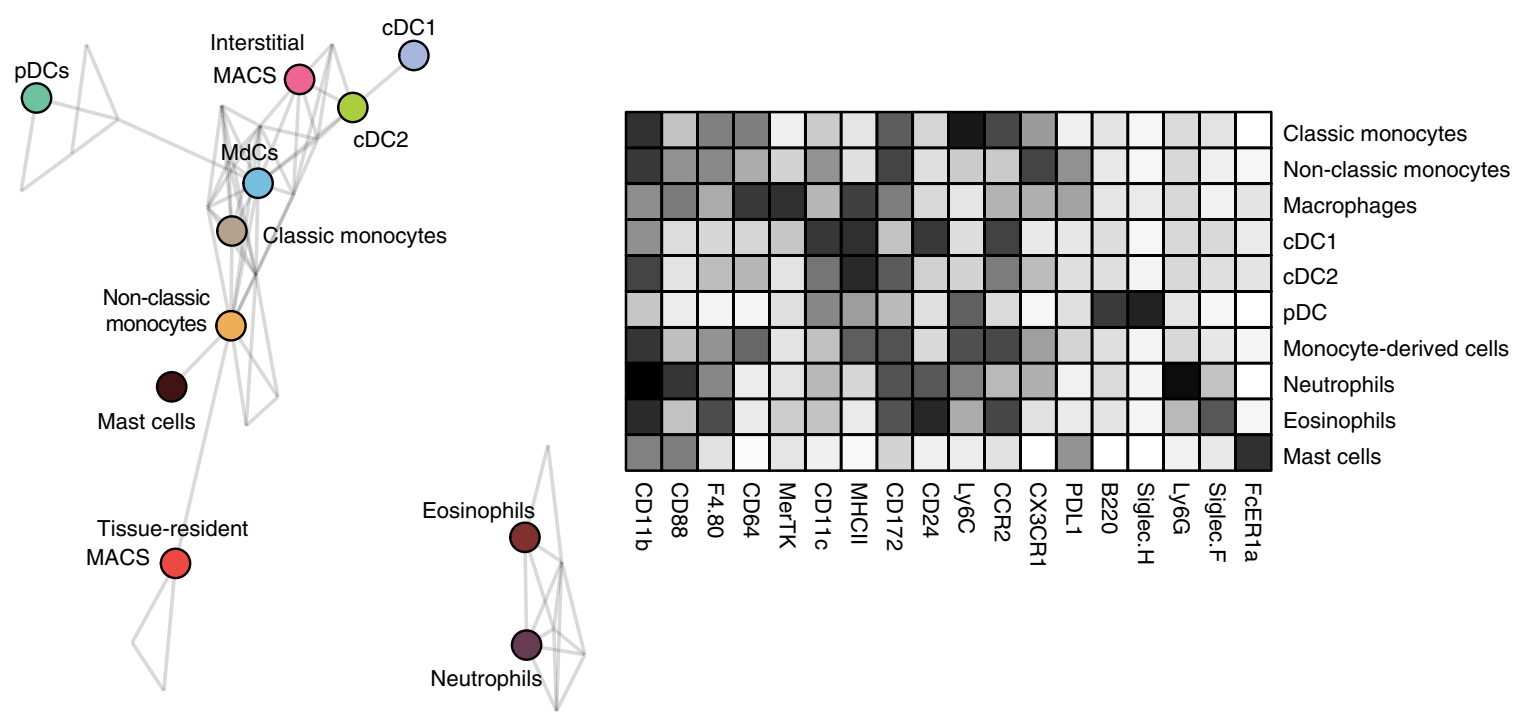
(for example $\gamma \delta \mathrm{T}$ and NKT cells) were twice as abundant in lungs from old versus young mice $(P=0.01)$. Taken together, we observed an overall reduction in canonical lymphocytes from the aging lung, compensated by an increase in quasi-innate lymphocytes during aging, that may then occupy the cellular niche of the departed $\alpha \beta$ T cells.

Alongside, we observed an overall increase in the frequency of total myeloid cells in the leukocyte fraction of lungs from aged mice (from $26.6 \%$ in young, to $41.6 \%, P=0.01$ ) (Fig. $2 \mathrm{c}, \mathrm{d}$ ). When we analyzed the composition of the myeloid compartment in the murine lung, we observed a significant decrease in frequency of cellular subsets associated with phagocytosis and debris clearance, as well as antigen processing and presentation, in aged compared to young mice: alveolar macrophages (termed AM, identified as CD11b CD11 $\mathrm{c}^{\text {high }}$ Siglec-Figh $)$ dropped from $8.0 \%$ to $1.6 \%(P<0.001)$ and eosinophils declined from $3.8 \%$ to $1.1 \%(P=0.013)$; while $\mathrm{cDC} 1$ dropped by almost half in the aged lung $(P=0.004)$. Furthermore, we also observed a significant drop of the frequency of pDCs $(P=0.0001)$-another common feature shared across a plethora of tissues (Fig. 2d).

Furthermore, our analysis also highlighted interesting phenotypic changes of these subpopulations: in particular, we saw a shift of AM within the dimensionality reduction representation (red cluster in Fig. 2c), that is mainly driven by an increased MHC-II expression on AMs in the aged lung (Fig. 2e). This phenotype suggests an increased MHC-II-mediated communication between $\mathrm{T}$ cells and tissue-resident macrophages, which occurs especially in inflammatory disease settings ${ }^{33,34}$. This may be also a result of interferon gamma (IFN- $\gamma$ ), a main driver of MHC-II expression ${ }^{35}$, usually presented by adaptive $\mathrm{T}$ cells in the lung microenvironment ${ }^{36}$. Our validation cohort acquired using spectral flow cytometry reflects the same age-related frequency changes within the myeloid fraction of the lung (Fig. 2f).

In summary, the aging lung reveals a decline in several immune compartments. Most notably, we identified a reduction in $\mathrm{T}$ helper cell as well as NK cell frequencies, which was accompanied by an increase in the total myeloid cell fraction. A general trend in loss of specialized tissue-resident macrophages ${ }^{37}$ as well as $\mathrm{DCs}^{38}$ was previously reported.

Aging induces organ-specific immune adaptations. To highlight the robustness of our analysis, we present the immune compartments of a further representative secondary lymphoid tissue (SLT) (sdLN) and soft connective tissue (VAT). In the former, we found a relative reduction in the NK $(P=0.05)$ and $\mathrm{T}$ helper cell $(P=0.003)$ fractions in aged compared to young mice (Fig. 3a,b). Simultaneously, and likely as a result of an empty cellular niche, we observed a relative increase in B cells $(P=0.06)$ within the sdLNs (Fig. 3b), thus confirming previous findings ${ }^{39}$. In the sdLNs we observed relative stability in total myeloid abundance (Fig. 3c), despite the reduced CD4 T cell and innate lymphoid cell numbers
(Fig. 3d). Within the myeloid compartment of the LNs, we once again observed a significant $(P=0.02)$ decline in the frequency of pDCs, as well as NK cells $(P=0.05)$. Taken together, whereas aging barrier tissues, such as the lung, showed a marked alteration within the tissue-resident and surveilling myeloid compartment, the overall abundance of total myeloid cells in the aging lymph nodes (LNs) remains relatively stable.

Initial dimensionality reduction visualization of the total $\mathrm{CD} 45^{+}$ cells isolated within the VAT (Fig. 3e) demonstrated again a significant reduction of the NK cell compartment $(P=0.015)$ as well as the tissue-resident innate lymphoid cell fraction $(P=0.015)$ from aged compared to young mice (Fig. 3f). We next enumerated the frequencies of the various myeloid cells present in the VAT (Fig. $3 g$,h). Of note, we found that while the total myeloid fraction was significantly reduced in the aging mouse $(P=0.05)$, the dynamics of various myeloid populations were stable within the VAT. Among myeloid cells, we did observe the emergence of a population of tissue-resident adipose tissue macrophages (termed Ly6C adipose tissue macrophages, ATMs) in the aged mice, which has previously been associated with obesity ${ }^{40}$ - consistent with increased susceptibility to obesity-related comorbidities in human aging ${ }^{41}$. While we have focused on the general age-related changes within these tissues for the purpose of this article, we have provided data acquired through the validation cohort (spectral flow cytometry) and sexrelated differences within Extended Data Figs. 3 and 4.

Age-induced lymphoid contraction is shared across tissues. We have applied a similar analysis pipeline-as presented for our representative organs-for all the tissues encompassed within our aging immune atlas. We assessed the frequency changes and their relative abundance in all the tissues, between sexes in aging. A summary of those features is presented within Extended Data Fig 4. We present an overview of the relative abundance of each immune population within these tissues, showcasing the richness of this resource (Fig. 4 and Extended Data Fig. 5a). We calculated the relative fold change in abundance of each identified immune population. To allow for schematic comparisons of the overall trends in lymphocyte counts between the two age groups, we employed a correlogram-style display in which the relative fold change in each population size between young and aged mice was mapped using a bubble chart, with the size of each circle representing the size of the cellular fold change (Fig. 5). The heterogeneity of the aging leukocyte trends makes it difficult to cluster tissues into their respective groups (barrier versus SLTs, among others). However, we have observed conserved trends in our aging model, presented as volcano plots (Extended Data Fig. 5b), where $P$ values can also be visualized. In the majority of assessed tissues, we observed a sustained and significant reduction in total CD4 T cell, NK cell and pDC compartments, and a general trend towards increased granulocytes in aged mice. Furthermore, while the loss of pDCs in the aging peripheral circulation is widely accepted ${ }^{42}$, we hereby demonstrate that this change

Fig. 2 | Aged lungs contain high frequencies of myeloid cells. a, UMAP with FlowSOM overlay showing total CD45 compartment of young adult and aged lungs. b. Violin plots ( $n=8$ young, $n=7$ aged) depicting frequencies of FlowSOM-generated immune cell clusters shown in $\mathbf{a}$. Statistical analysis was carried out using nonparametric Mann-Whitney-Wilcoxon tests. The $P$ value was then adjusted for multiple comparisons using a BH test. $P$ values of less than 0.05 were considered significant and are indicated by the numerical value on the respective graphs. $\mathbf{c}$, UMAP with FlowSOM overlay showing total myeloid compartment (as shown in a, dark blue cluster) of young adult and aged lungs. d, Violin plots ( $n=8$ young, $n=7$ aged) depicting frequencies of FlowSOM-generated immune cell clusters shown in c. All depicted $P$ values are significant $(<0.05)$ with a $\mathrm{BH}$ false discovery rate of less than $5 \%$. e, Dot plots showing the gating strategy of alveolar macrophages (left panel). Histogram and subsequent heatmap depicting differential median marker expression in alveolar macrophages of young adult and aged lungs (two right-most panels). Statistical analysis was carried out using nonparametric Mann-Whitney-Wilcoxon tests. The $P$ value was then adjusted for multiple comparisons using a $\mathrm{BH}$ test. $P$ values of less than 0.05 were considered significant and are indicated by the numerical value on the respective graphs. $\mathbf{f}$, Violin plots ( $n=10$ young, $n=10$ aged) depicting frequencies of immune cell clusters acquired through the validation FACS cohort. Statistical analysis was carried out using nonparametric Mann-Whitney-Wilcoxon tests. The $P$ value was then adjusted for multiple comparisons using a $\mathrm{BH}$ test. $P$ values of less than 0.05 were considered significant and are indicated by the numerical value on the respective graphs. Also see details in Extended Data Fig. 2. 
is sustained across a wide range of aged tissues. Taken together, the loss of NK cells and pDCs in the aging lung may result in reduced and/or delayed viral clearance.
Aging induces loss of CD103 in naive CD8 T cells. Mass-cytometry analysis of the aging mouse has allowed for the extensive dissection of the tissue-resident myeloid compartment with a resolution thus

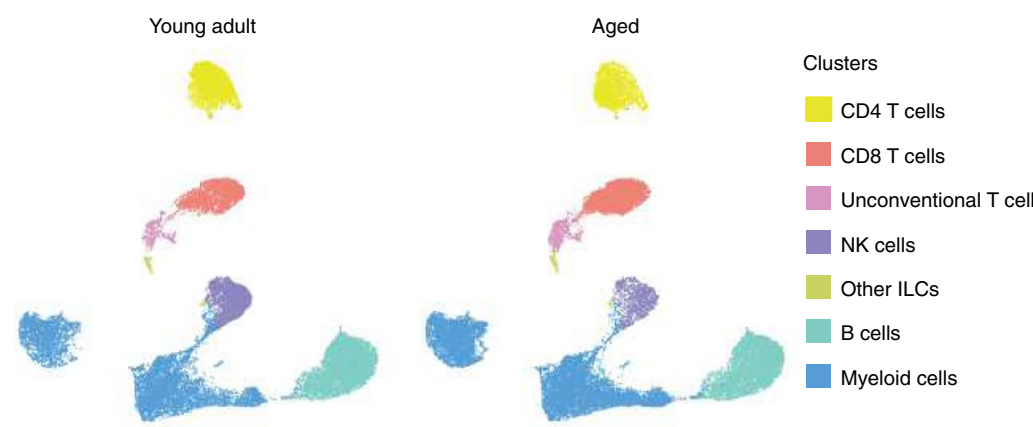

C

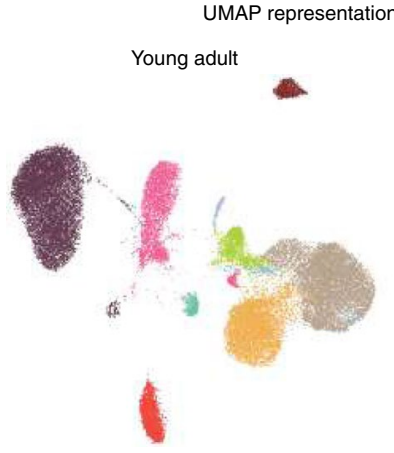

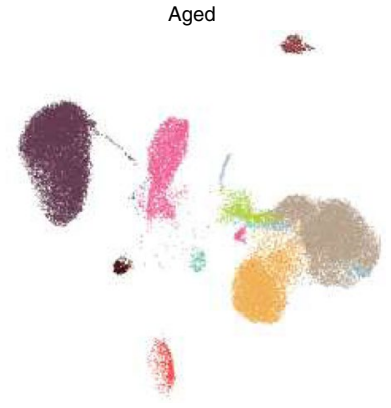

b Frequencies of lymphoid populations

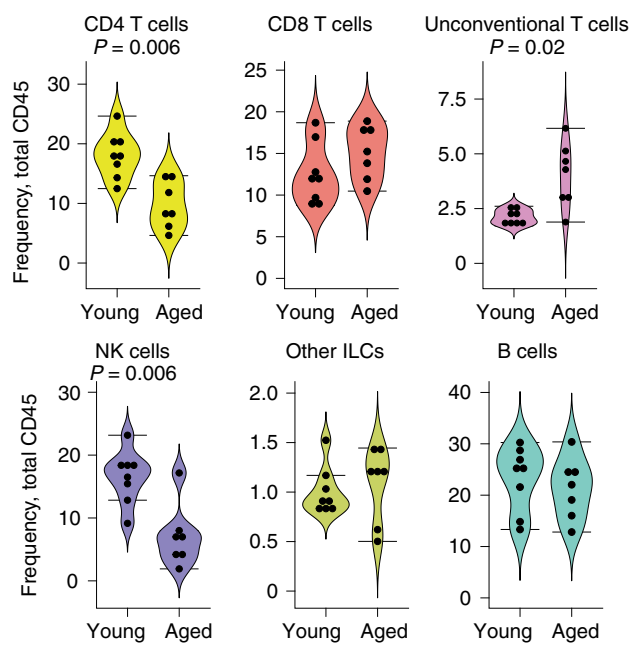

Clusters

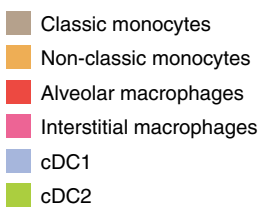

pDCs

MdCs

Neutrophils

Eosinophils

Mast cells

d

Frequencies of myeloid populations in lung

$\begin{array}{llll}\text { Myeloid cells } & \text { Classic } & \text { Non-classic } & \text { Alveolar }\end{array}$ monocytes monocytes macrophages
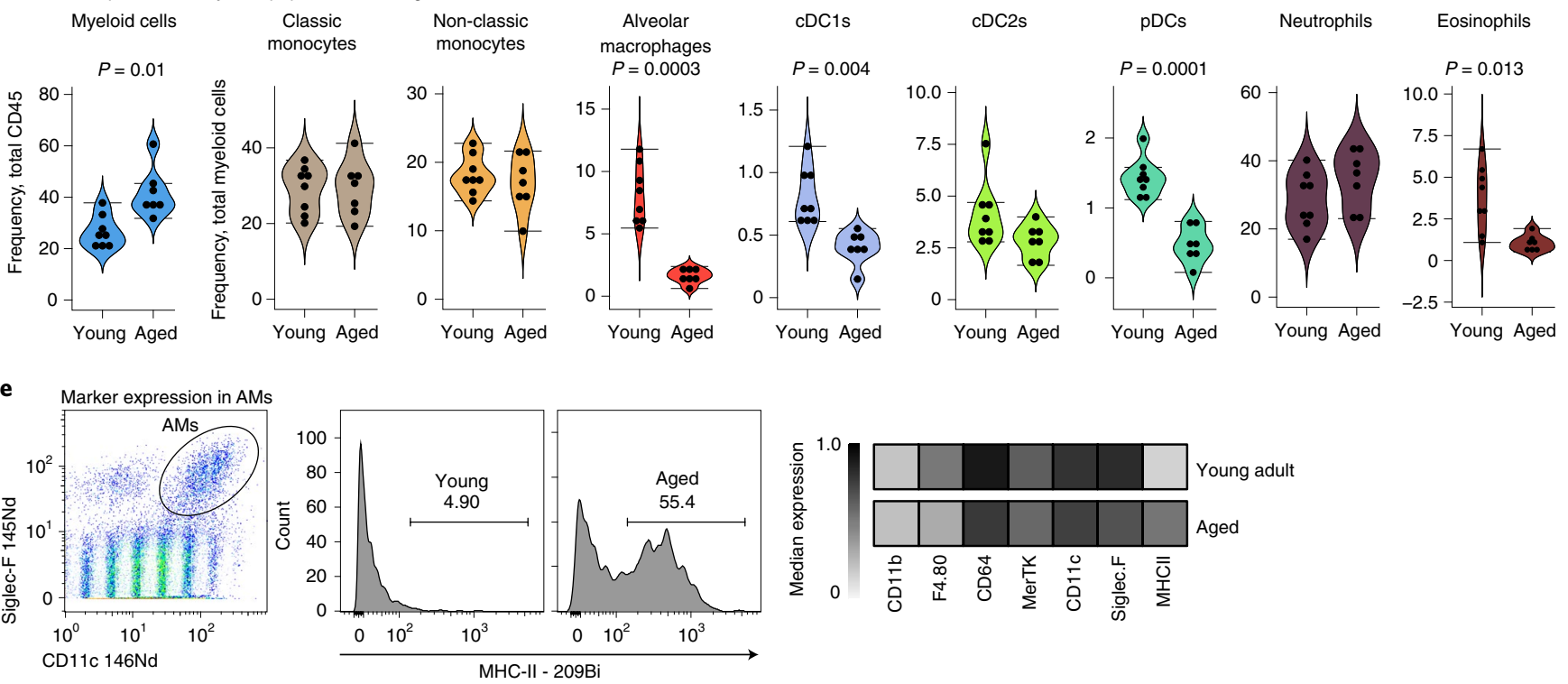

$\mathbf{f}$

Validation cohort FACS-derived myeloid frequencies
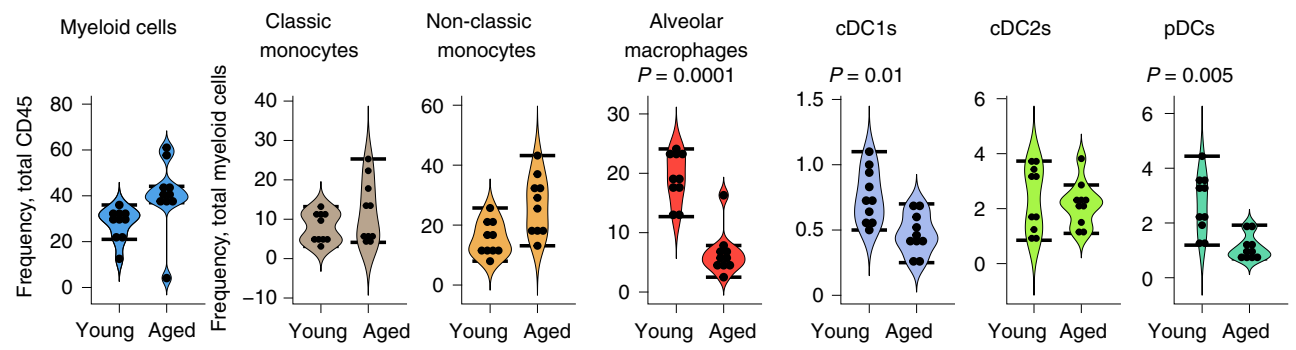
a

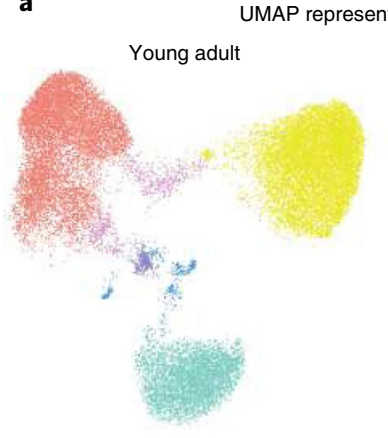

c

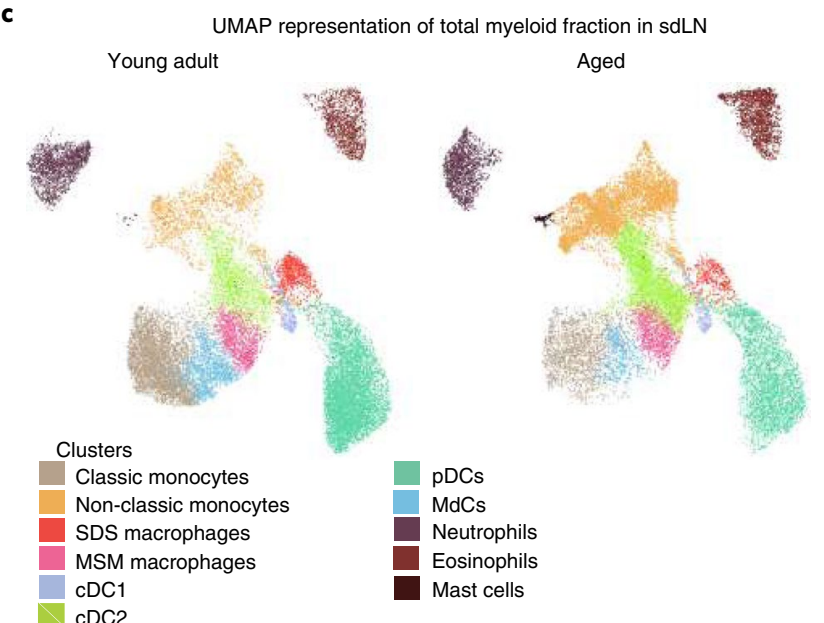

b

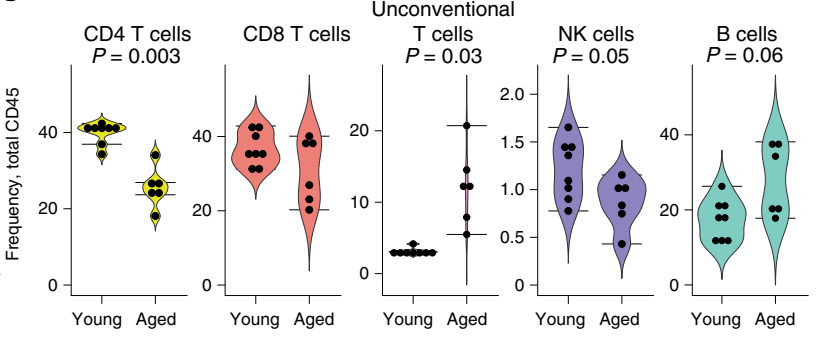

e

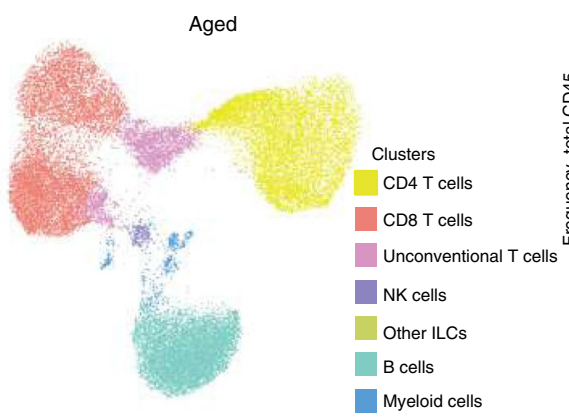

d
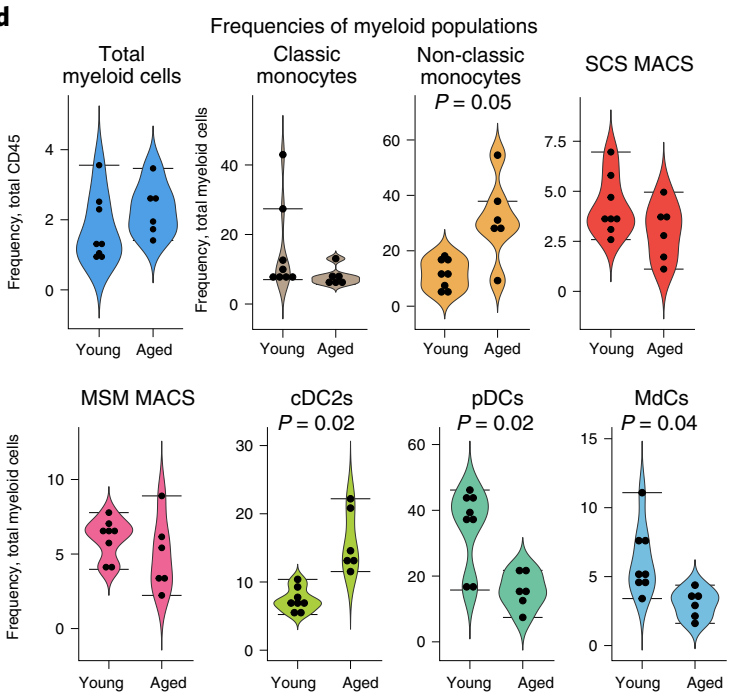

UMAP representation of total CD45 fraction in VAT
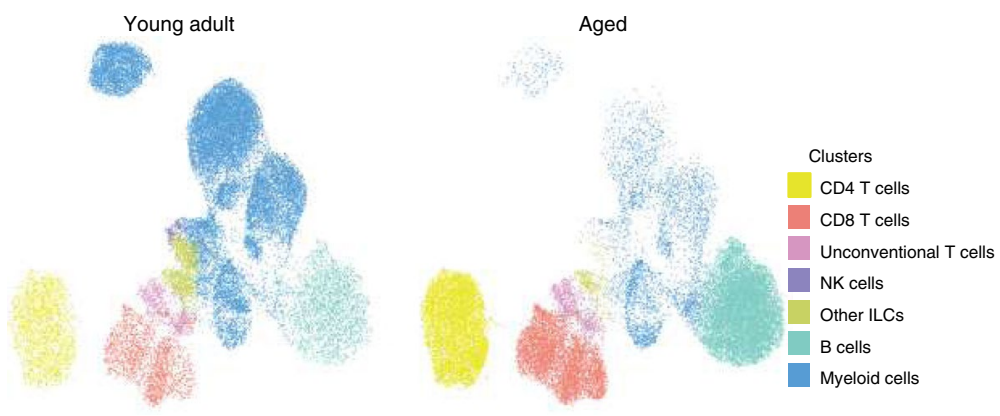

g

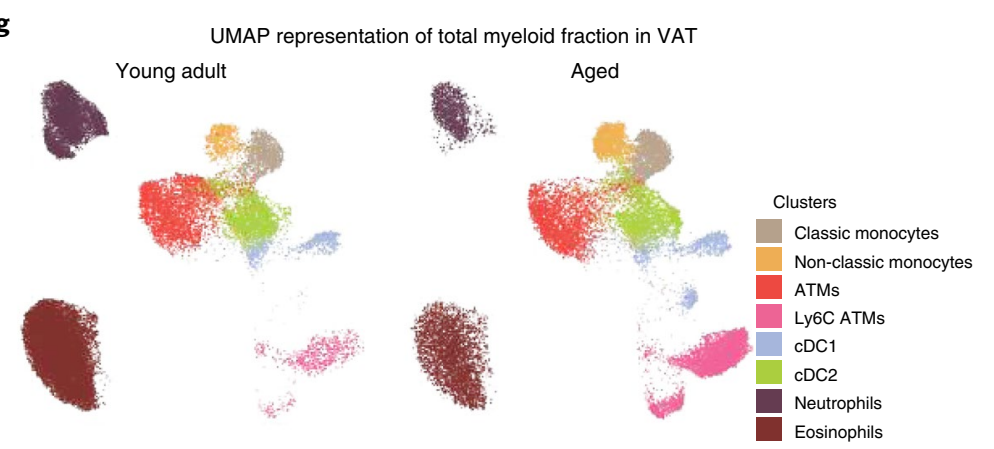

\section{f}

Frequencies of lymphoid populations

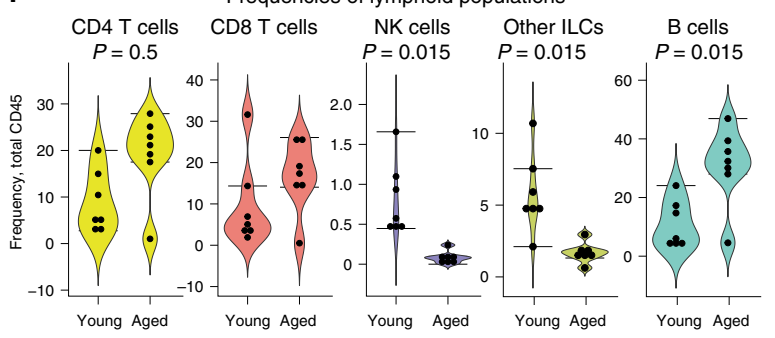

h
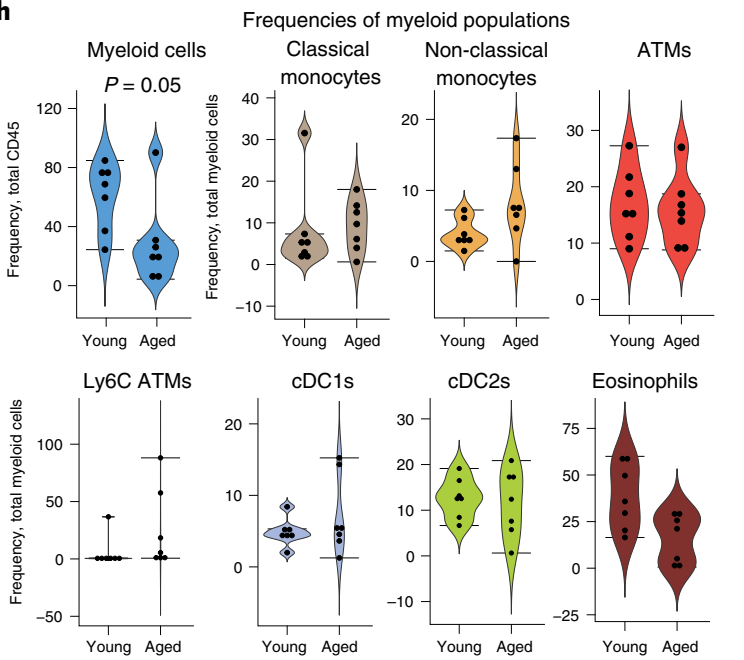

far not achieved using conventional flow-cytometry methods. To delineate further the lymphoid compartment, we acquired additional cohorts of age-matched mice using spectral flow cytometry, using a specifically designed antibody panel. To demonstrate the synergy between the two flow-cytometry experimental batches, the effect size (ES) was computed. This is a statistical measure whereby the median expression of each measured marker was calculated for each immune population (as listed in Extended Data Fig. 6a) per tissue 
Fig. 3 | Aged LNs exhibit contraction of CD4 T cell, pDC and NK cell populations, while VAT presents a stable myeloid compartment after aging. a, UMAP with FlowSOM overlay showing total CD45 compartment of young adult and aged sdLN. b, Violin plots ( $n=8$ young, $n=7$ aged) depicting frequencies of FlowSOM-generated immune cell clusters shown in a. Statistical analysis was carried out using nonparametric Mann-Whitney-Wilcoxon tests. The $P$ value was then adjusted for multiple comparisons using a $\mathrm{BH}$ test. $P$ values of less than 0.05 were considered significant and are indicated by the numerical value on the respective graphs. c, UMAP with FlowSOM overlay showing total myeloid compartment (as shown in a, dark blue cluster) of young adult and aged SdLN. d, Violin plots ( $n=8$ young, $n=7$ aged) depicting frequencies of FlowSOM-generated immune cell clusters shown in $\mathbf{c}$. Statistical analysis was carried out using nonparametric Mann-Whitney-Wilcoxon tests. The $P$ value was then adjusted for multiple comparisons using a $\mathrm{BH}$ test. $P$ values of less than 0.05 were considered significant and are indicated by the numerical value on the respective graphs. e, UMAP with FlowSOM overlay showing total CD45 compartment of young adult and aged VAT. f, Violin plots ( $n=8$ young, $n=7$ aged) depicting frequencies of FlowSOMgenerated immune cell clusters shown in e. Statistical analysis was carried out using nonparametric Mann-Whitney-Wilcoxon tests. The $P$ value was then adjusted for multiple comparisons using a BH test. $P$ values of less than 0.05 were considered significant and are indicated by the numerical value on the respective graphs. $\mathbf{g}$, UMAP with FlowSOM overlay showing total myeloid compartment (as shown in $\mathbf{e}$, dark blue cluster) of young adult and aged VAT. $\mathbf{h}$, Violin plots ( $n=8$ young, $n=7$ aged) depicting frequencies of FlowSOM-generated immune cell clusters shown in $\mathbf{g}$. Statistical analysis was carried out using nonparametric Mann-Whitney-Wilcoxon tests. The $P$ value was then adjusted for multiple comparisons using a $\mathrm{BH}$ test. $P$ values of less than 0.05 were considered significant and are indicated by the numerical value on the respective graphs. Also see details in Extended Data Fig. 3.

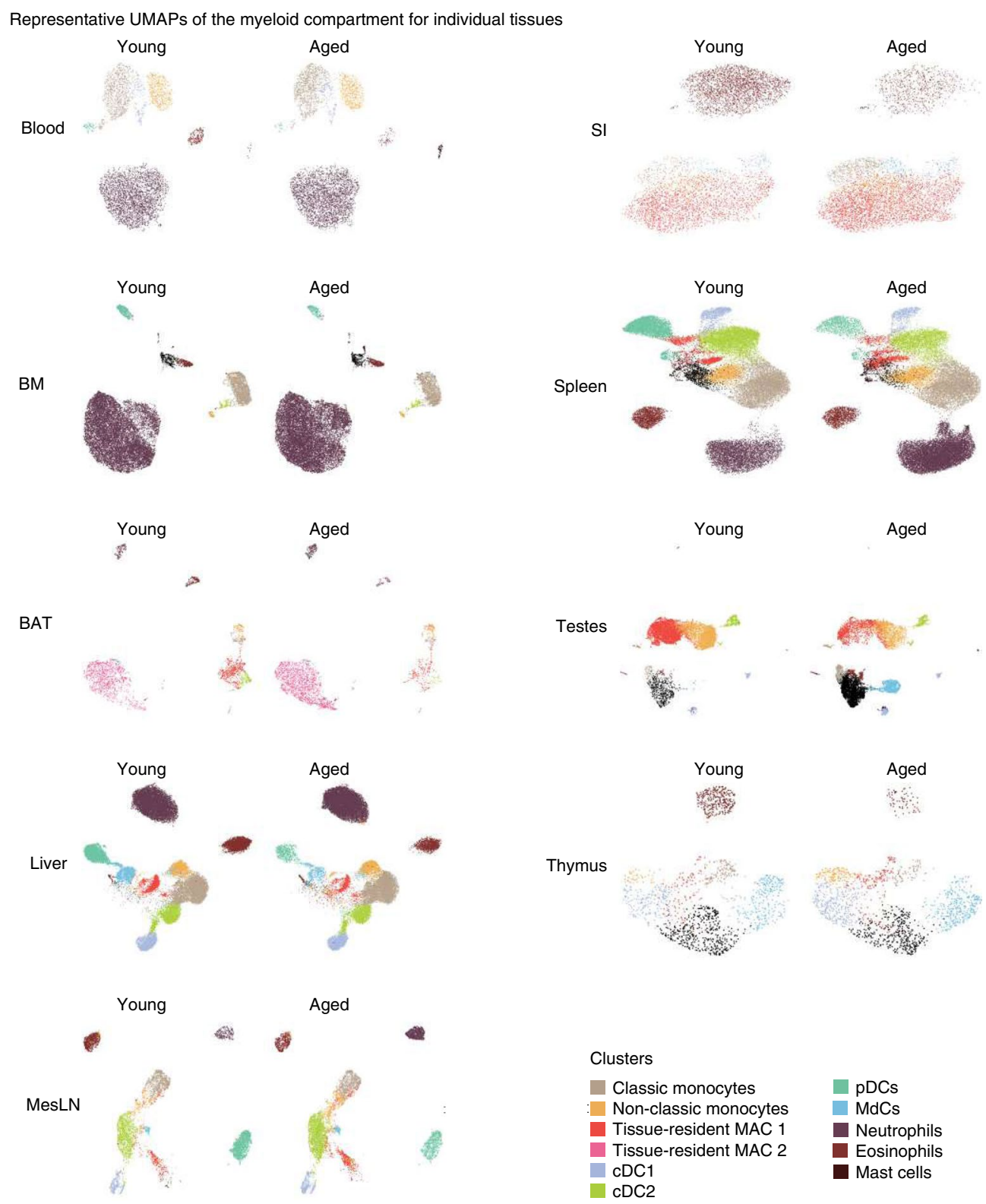

Fig. 4 | Comprehensive analysis of myeloid compartments was incorporated for all 12 tissues within immune atlas of aging. UMAP with FlowSOM overlay showing total myeloid compartment of young adult and aged tissues. A further nine tissues have been extensively analyzed using the pipeline presented in Figs. 2 and 3. Also see details in Extended Data Fig. 5 a. 


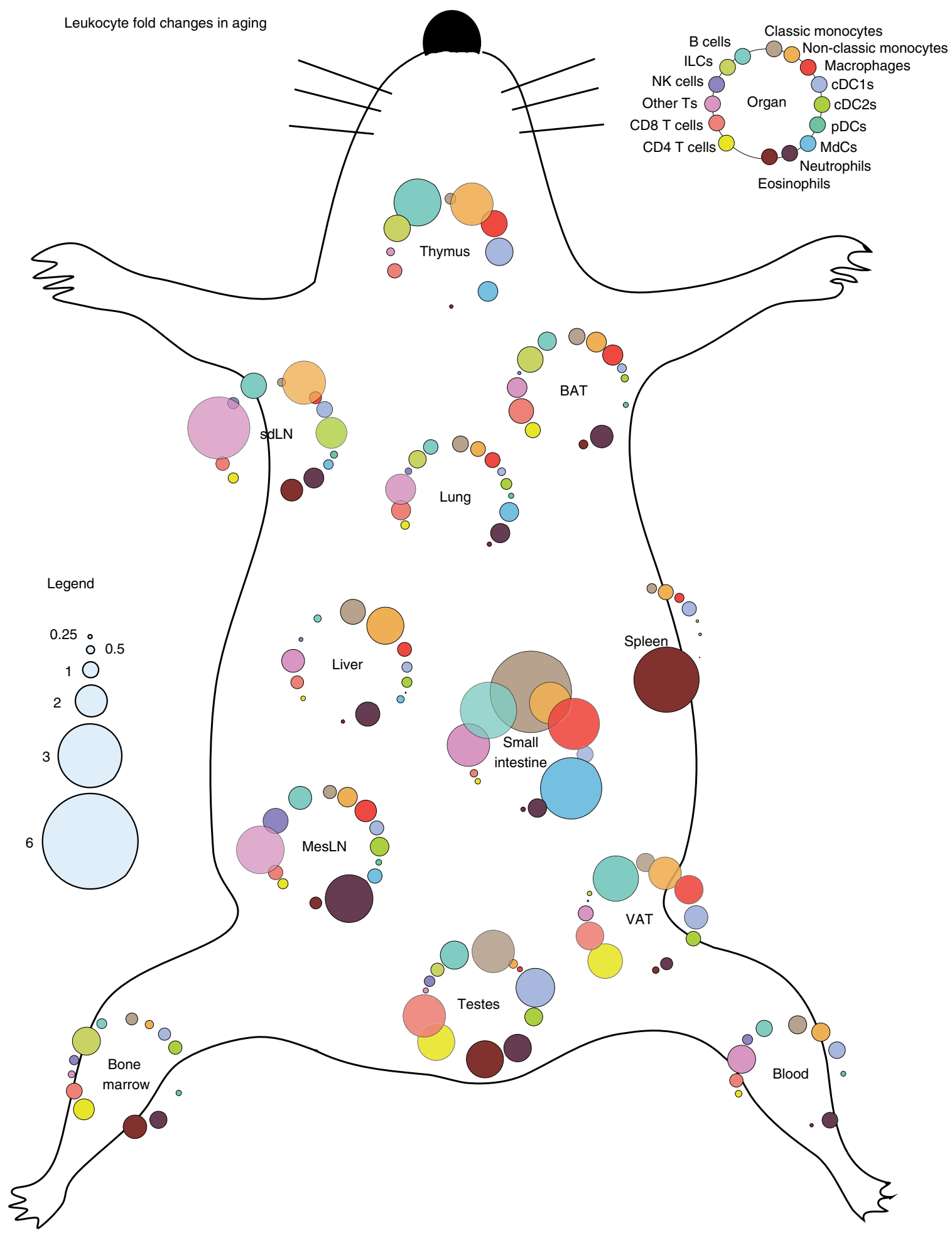

Fig. $\mathbf{5}$ | Schematic representation of fold changes in leukocyte frequencies in aged compared to young mice. Bubble chart of fold change in immune cell populations in aged relative to young mice (data averaged from analysis of $n=8$ young, $n=7$ aged mice). For each leukocyte subset, FlowSOM-derived frequencies of the young adult mouse were normalized to 1, and the subsequent fold change in the aged mouse was used to calculate bubble radius. Also see details in Extended Data Fig. 5b.

and per mouse, as well as the statistical significance of this feature between the two groups. The resultant plot combines both the significance as well as the median change for each feature (Fig. $6 \mathrm{a})^{43}$. With the majority of data points largely following the $R=1$ correlation, we confirm that our two datasets generated through spectral flow cytometry are highly consistent. By introducing a cut-off value for the ES as described by Cohen ${ }^{44}$, we screened for hits that were significant by setting a threshold of 2.5 on both axes, to find phenotypic changes that are associated with aging, consistent between the batches and tissue-wide (Fig. 6a). Upon screening the hits, we noted a crisp, significant reduction in CD103 expression within the naive tissue-resident CD8 $\mathrm{T}$ cell compartment of the majority of tissues 
analyzed. The feature was common among SLTs and non-lymphoid tissues alike (Fig. 6b). In tissues where this change was not significant, we observed a clear trend (Extended Data Fig. 6b). The stability of the naive CD8 T cell frequency among all the tissues analyzed (Fig. 6c) suggests that this is a phenotypic, age-related adaptation.

Our validation data show that the homogeneous drop in CD4 $\mathrm{T}$ cell frequencies is largely driven by a sustained loss of naive CD4 $\mathrm{T}$ cells in aged tissues, as opposed to CD4 cell memory subsets (Fig. 6d). Altogether these findings underline the numerical and functional impairment of the naive $\mathrm{T}$ cell compartment in aging.

Aged bone marrow exhibits early signs of myeloid bias. One potential mechanism that could underpin many of the changes described within our resource is an altered, age-driven hematopoiesis. The myeloid lineage-commitment bias in aging ${ }^{45,46}$ is associated with declining immunocompetence and increased autoimmunity observed in the aged mouse $e^{47}$. We sought to investigate whether the overall tissue-wide reduction in the lymphocyte compartment was reflected in the early stages of hematopoiesis in the bone marrow of young adult and aged mice. We interrogated the hematopoietic stem and progenitor cells in the BM via spectral flow cytometrythrough a 24 -color surface marker panel to identify 35 clusters encompassing all known hematopoietic progenitor subsets, while also interrogating the mature $\mathrm{CD} 45^{\text {pos }}$ cells within the BM (Fig. 7a). We classified the cells according to their marker expression: lymphoid cells, identified using a lineage channel (Lin $\left.{ }^{\text {high }}\right)$; mononuclear phagocytes (Ly6G ${ }^{\text {low }} \mathrm{CD} 11 \mathrm{~b}^{\text {high }}$ and/or $\mathrm{CD} 11 \mathrm{c}^{\text {high }}$ ); pDCs (Lin ${ }^{\text {high }}$ Siglec- $\left.\mathrm{H}^{\text {high }}\right)$; preneutrophils (preNeu, CD11 $\mathrm{b}^{\text {high }} \mathrm{CD} 117^{\text {high }}$ ); neutrophils (Ly6 $\left.\mathrm{G}^{\text {high }}\right)$; eosinophils ( $\left.\mathrm{SSC}^{\text {high }}\right)$; basophils $\left(\mathrm{Fc \varepsilon} \mathrm{R}^{\text {high }}\right)$; and progenitors (Lin ${ }^{\text {low }} \mathrm{Ly} 6 \mathrm{G}^{\text {low }} \mathrm{CD} 11 \mathrm{~b}^{\text {low }} \mathrm{CD} 117^{\text {high }}$ ) (see Extended Data Fig. 7a-c). Notably, we found a significant decrease in lymphoid cells, complemented by a significant increase in preNeu (Extended Data Fig. 7d,e), suggestive of an increase in neutrophil output. To assess the complex cocktail of HSC subsets and progenitor frequencies in the BM, we visualized the progenitor compartment using a force-directed layout ${ }^{48}$, overlaid with FlowSOM-derived clusters (Fig. 7b). This strategy allowed us to identify, on the basis of the median expression of all markers depicted in the heatmap (Fig. 7c), 19 diverse progenitor subsets including long-term hematopoietic stem cell (LT-HSC) and short-term HSC subsets; multipotent progenitors found in the so-called LSK fraction (defined by their c-kit and Sca-1 expression and by the absence of lineage marker expression); common lymphoid progenitors (CLPs); megakaryocyte and erythrocyte progenitors; common myeloid progenitors; granulocyte and monocyte progenitors (GMPs); monocyte and DC progenitors (Fig. 7c).

Among the LSK subsets, we observed a significant increase in LT-HSC frequency $(P=0.005)$ in BM from aged compared to young mice (Fig. 7 d). While we observed only a trend towards common myeloid progenitors and GMPs, we measured a significant increase in GMP-committed progenitor fractions, namely proNeul and
$\mathrm{cMoP}^{49}(P<0.05)$, paralleled by a clear trend towards CLP reduction in aged compared to young mice (Fig. $7 d$ ). To characterize the impairment and specific age-related alteration in CLP commitment, as well as to increase the resolution on these rare progenitors, we used manual gating and we further focused on $\mathrm{Lin}^{-} \mathrm{CD} 117^{\text {low }} \mathrm{Sca}-$ $1^{\text {low }} \mathrm{CD} 135^{\text {high }} \mathrm{CD} 127^{\text {high }}$ CLP. Here, using two markers, we could characterize Siglec- $\mathrm{H}^{\text {low }}$ Ly6D ${ }^{\text {low }}$ T/NK cell, Siglec- $\mathrm{H}^{\text {low }}$ Ly6 ${ }^{\text {high }} \mathrm{B}$ cell and Siglec- $\mathrm{H}^{\text {high }}$ Ly6 $\mathrm{D}^{\text {high }} \mathrm{pDC}$ bona fide committed progenitors ${ }^{50}$ (Extended Data Fig. 7f). In line with our unbiased high-dimensional analysis showing a general impairment of lymphopoiesis, we found a significant reduction of all three lineage-committed CLP fractions in aged mice (Fig. 7 e, T/NK precursors $\mathrm{P}<0.05$; $\mathrm{B}$ precursors $P<0.0001$; pDC precursors $P<0.0001$ ). This, together with the increase in myeloid-committed progenitors, provides strong evidence for a skewing towards myelopoiesis during aging at the cost of a contracting lymphopoiesis. Similarly, the loss of pDCs is a feature observed across most of the other tissues analyzed in our study, which may be explained by the unique ontogeny of these cells, emerging, at least in part, from the reduced Siglec- $\mathrm{H}^{\text {high }} \mathrm{Ly} 6 \mathrm{D}^{\text {high }}$ fraction of the $\mathrm{CLP}^{50}$.

Our second spectral flow-cytometry cohort validated the abovedescribed age-related changes to lineage commitment and was used to power a more statistics-driven, unbiased analysis.

LT-HSC contraction correlates with aged tissue-resident lymphocytes. To uncover rare age-associated immune features independent of traditional leukocyte nomenclature, we carried out an indepth analysis of BM progenitors from two independent spectral flow cohorts using supervised representation learning (CellCNN) approaches followed by FlowSOM-based validation ${ }^{51}$. This allowed the convergent identification of a small cellular subset of $\mathrm{Lin}^{-} \mathrm{SCA} 1^{+} \mathrm{CD} 48^{-} \mathrm{CD} 150^{+}$LT-HSCs, which was expanded in the aging BM (Fig. $8 \mathrm{a}$ and Extended Data Fig. 7). The FlowSOMidentified matching LT-HSC cluster from cohort 1 showed an overlapping signature and comparable frequency among progenitors, being significantly enriched in aging (Fig. $8 \mathrm{~b}$ and Extended Data Fig. 7). We observed that the median expression of CD34 was significantly decreased in the old group (Fig. 8c), suggestive of reduced mobilizing and repopulating activity of aged LT-HSC ${ }^{52,53}$.

We then computed the correlation between age-affected BM progenitor subsets and their mature lymphoid counterparts, by considering the frequencies of each subset as per each tissue analyzed (Fig. 8d,e). We observed widespread and significant age-induced changes in the relationship between HSCs and lymphoid-committed progenitors with the peripheral adaptive immune populations. In particular, as exemplified by lung and sdLN lymphocytes (Fig. 8c,d) in aging, we observed a positive correlation between the $\mathrm{BM}$ progenitors and systemic lymphoid compartment cell frequencies. Alltogether, these observations strengthen the concept that lymphoid progenitor depauperation in the hematopoietic niche is the substrate for peripheral lymphocyte contraction during aging.

Fig. 6 | Spectral flow-cytometry analysis of tissue-wide tissue-resident compartment of aged mice revealed phenotypic change in naive CD8 T cells and atrophy of naive CD4 T cell compartment. a, Comparison of immune features derived from each leukocyte subpopulation between experimental groups. Dot plot displaying the ES calculated between young versus old mice in validation cohort batch 1 ( $n=10$ young, $n=10$ aged) ( $x$ axis) compared with the ES calculated in the same in validation cohort batch 2 ( $n=10$ young, $n=10$ aged) ( $y$ axis). Each dot represents one immunological feature; colors represent individual tissues. $\mathbf{b}$, Violin plots depicting the median expression of CD103 in naive CD8 T cells of young and aged mice from both validation cohorts ( $n=5$ young, $n=5$ aged from cohort $1 ; n=5$ young, $n=5$ middle, $n=5$ aged from cohort 2). All depicted changes from young/middle to aged are significant ( $<0.05$ ) with a $\mathrm{BH}$ false discovery rate of less than $5 \%$. c, Violin plots depicting the frequencies of naive CD8 $\mathrm{T}$ cells of young and aged mice from both validation cohorts ( $n=10$ young, $n=10$ aged from cohort 1 . For cohort $2, n=5$ young, $n=5$ middle, $n=5$ aged for liver, spleen, VAT, lung, blood and heart. For cohort 2, $n=10$ young, $n=10$ middle, $n=10$ aged for colon, MesLN, sdLN and spleen). d, Violin plots depicting the frequencies of naive CD4 T cells of young and aged mice from both validation cohorts ( $n=10$ young, $n=10$ aged from cohort 1 . For cohort $2, n=5$ young, $n=5$ middle, $n=5$ aged for liver, spleen, VAT, lung, blood and heart. For cohort 2, $n=10$ young, $n=10$ middle, $n=10$ aged for colon, MesLN, sdLN and spleen). Statistical analysis was carried out using nonparametric Mann-Whitney-Wilcoxon tests. The $P$ value was then adjusted for multiple comparisons using a BH test. $P$ values of less than 0.05 were considered significant and are indicated by the numerical value on the respective graphs. Also see details in Extended Data Fig. 6 . 


\section{Discussion}

As a consequence of our increasingly aged global population, there is a need to study the challenges faced by the immune systems of older adults. A rising number of research studies have focused on the immune landscape surrounding various age-related comorbidities. In humans, cellular analysis has often been limited to the
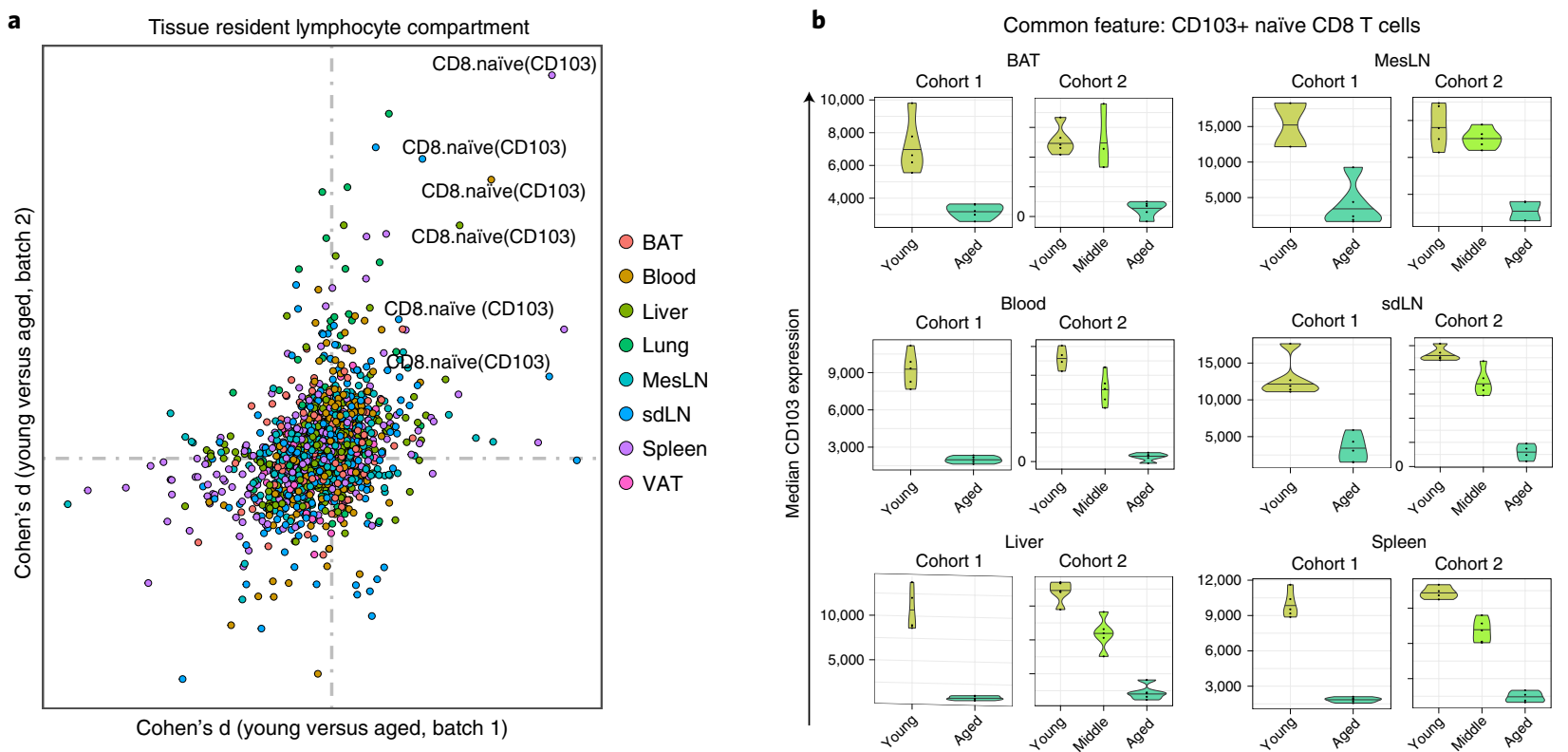

C Naïve CD8 T-cell frequencies
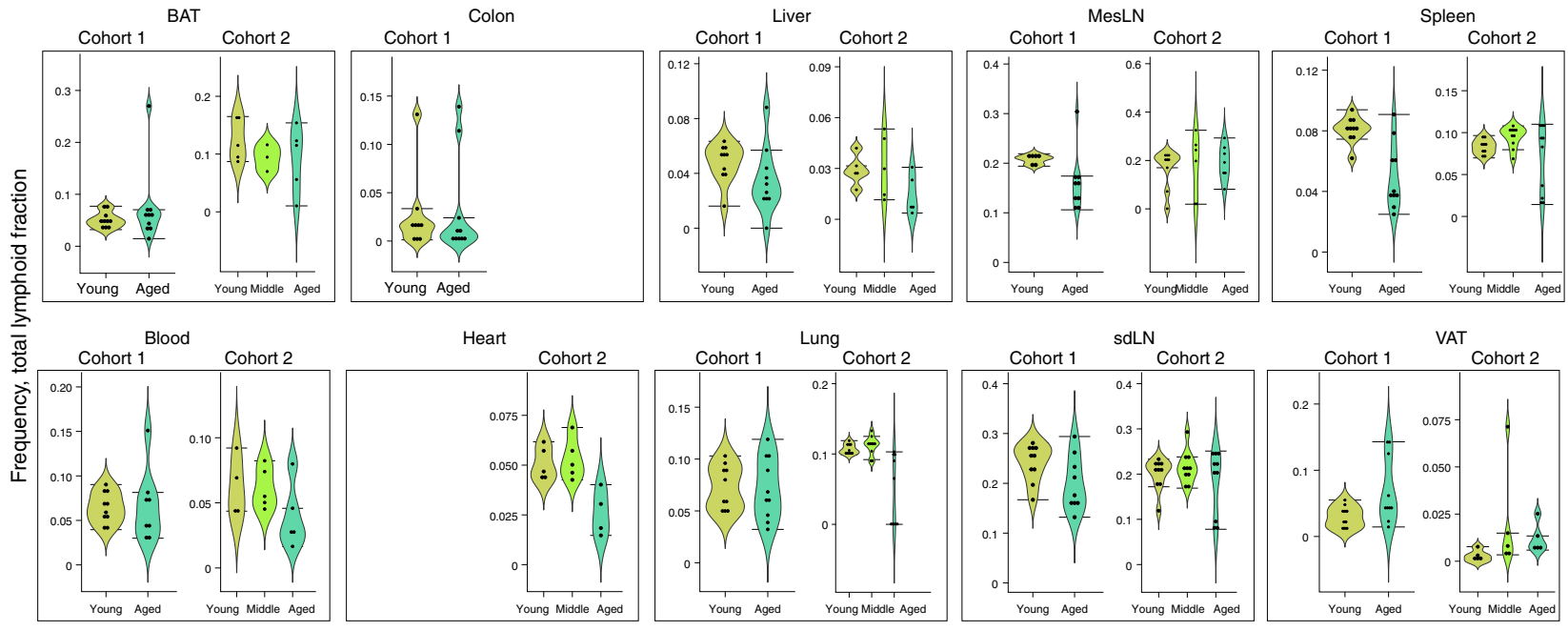

d Naïve CD4 T-cell frequencies
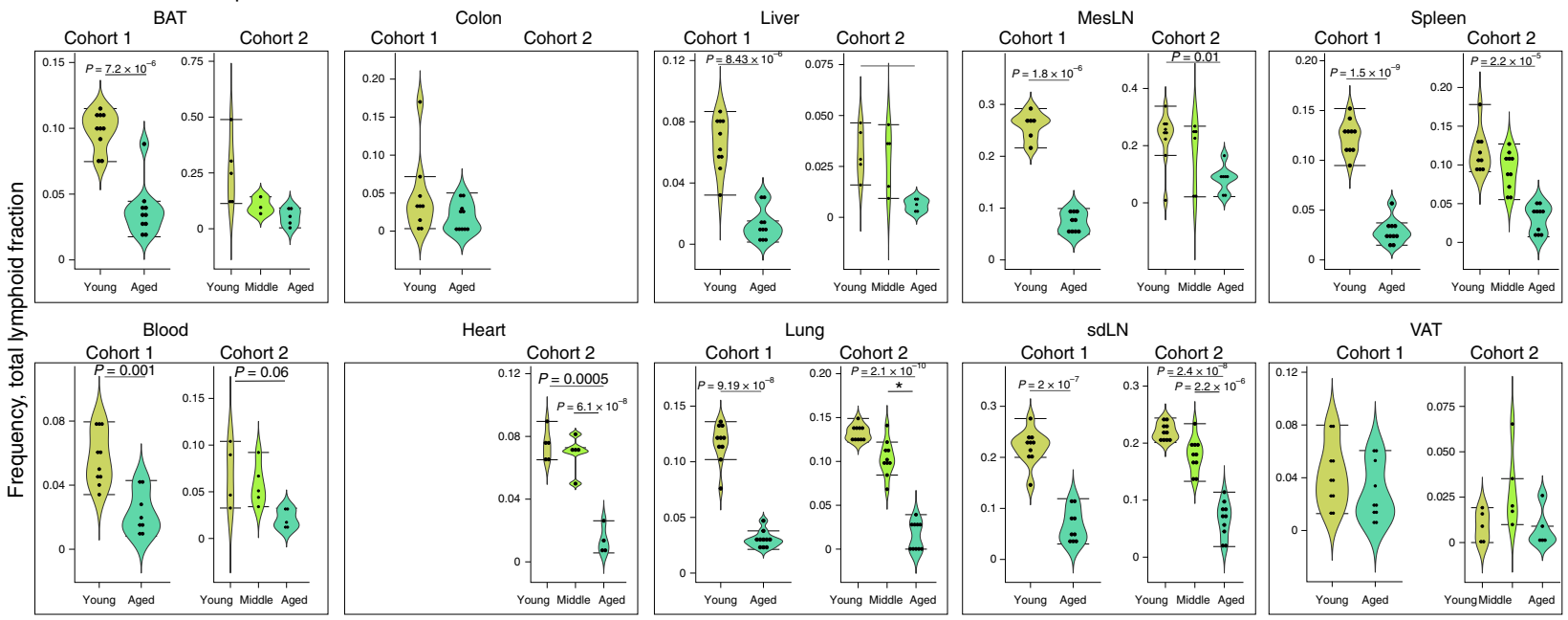
Scaffold map of all identified populations in BM

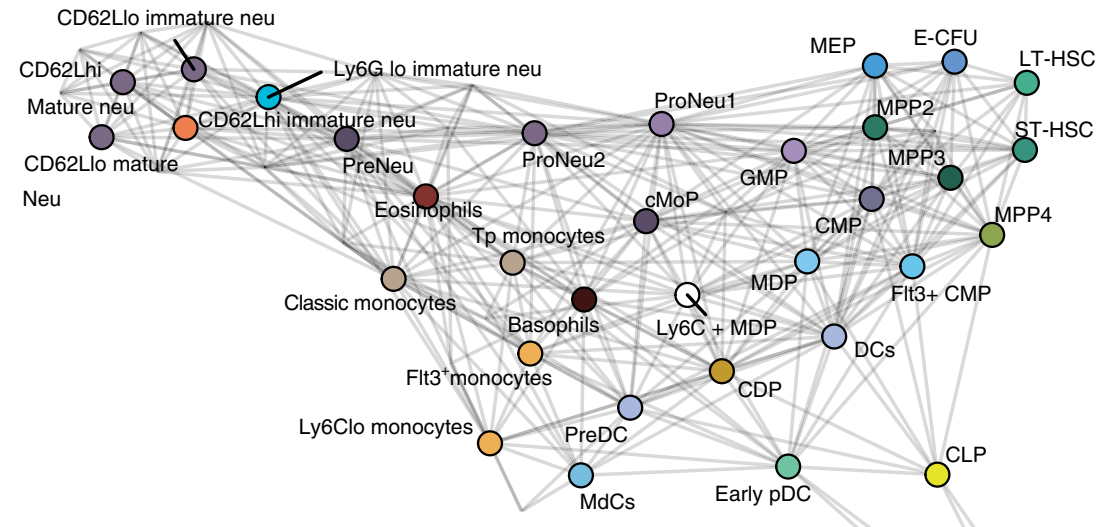

b

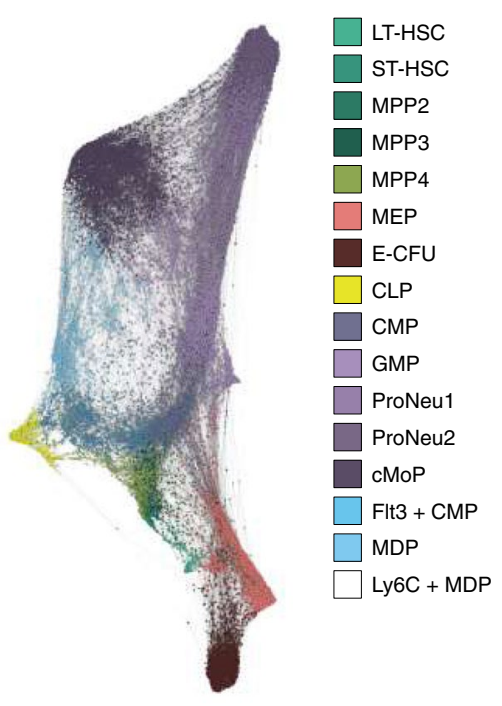

\section{d}

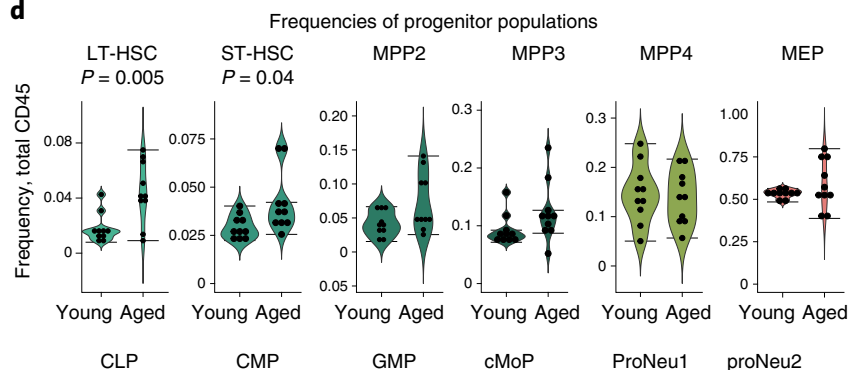

CLP

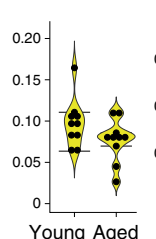

$$
P=0.04
$$$$
P=0.04
$$

proNeu2

Young Aged
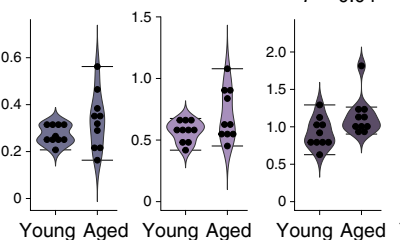

Young Aged Young Aged

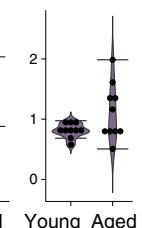

c

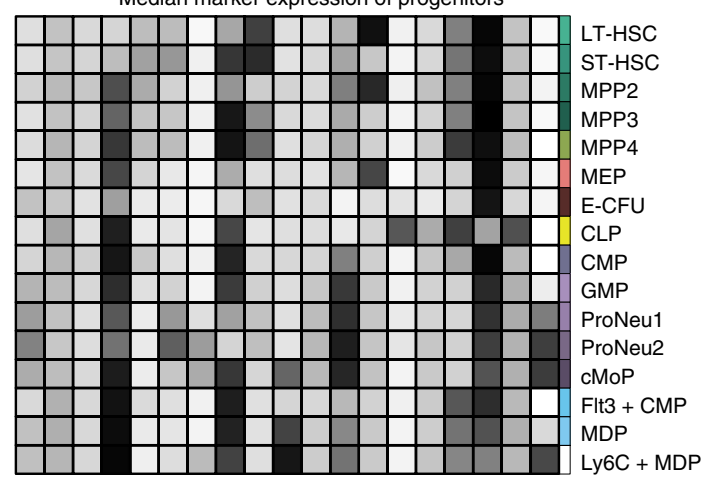

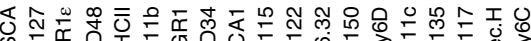

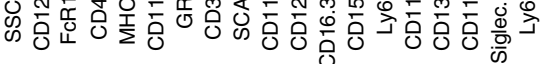

e

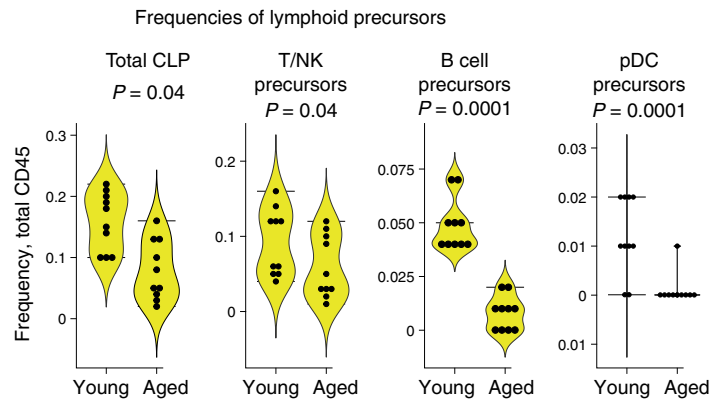

Fig. 7 | Hematopoietic compartment of aged murine bone marrow reveals contraction of lymphoid progenitors and skewing towards myelopoiesis. a, Modified scaffold map of the total CD45 ${ }^{\text {pos }}$ compartment of BM. b. Force-directed layout of combined young and aged progenitor clusters identified in part a. Colors depict FlowSOM-generated clusters. c, Heatmap depicting median marker expression in FlowSOM-derived progenitor subsets of the bone marrow. d, Violin plots ( $n=10$ young, $n=10$ aged) depicting frequencies among total CD45 ${ }^{\text {pos }}$ cells of FlowSOM-generated immune cell clusters shown in c. Statistical analysis was carried out using nonparametric Mann-Whitney-Wilcoxon tests. The $P$ value was then adjusted for multiple comparisons using a $\mathrm{BH}$ test. $P$ values of less than 0.05 were considered significant and are indicated by the numerical value on the respective graphs. $\mathbf{e}, \mathrm{Violin}$ plots $(n=10$ young, $n=10$ aged) depicting frequencies derived from manual gating of CLPs and downstream precursor subsets of the BM, subdivided on the basis of Siglec-H and Ly6D expression. Statistical analysis was carried out using nonparametric Mann-Whitney-Wilcoxon tests. The $P$ value was then adjusted for multiple comparisons using a $\mathrm{BH}$ test. $P$ values of less than 0.05 were considered significant and are indicated by the numerical value on the respective graphs. Also see details in Extended Data Fig. 7. 
leukocytes harvested from peripheral blood ${ }^{54,55}$ and/or focused on single organs or cell types ${ }^{56}$. Consequently, dynamic changes in the frequencies of immune populations have been largely overlooked. The recently published Tabula muris senis ${ }^{57,58}$ is a powerful tool for the interrogation of the subtle changes in the expression of various genes within aging mouse tissues. However, such a resource does not lend itself automatically to further interrogation through routine experimental procedures, for example basic flow cytometry. Our highly complex multiparameter panels have been designed specifically to capture the tissue immune compartment and to provide a rich immune atlas. Similarly, we captured the highly complex HSC compartment and its dynamic changes over two time points. The resulting immune atlas and its annotations lend themselves to further adaptation for routine lab procedures.

Here we present a comprehensive atlas of the young and aged murine immune system with the aim of highlighting patterns of change in immune cell types. We provide a major informational database, in which we have assessed the aging immune compartment of the C57/BL6 mouse, the mouse strain most commonly used in immunology-related research ${ }^{59}$. We aimed to provide a comprehensive analysis of the mouse model by assessing a diverse range of tissues and organs simultaneously, using an accessible protocol and mice commercially available to the research community. To assess the effects of aging on the immune compartment of the geriatric mouse model, we have powered our experiments adequately (with a combined total of $n=18$ young versus $n=17$ aged mice).

There is, unfortunately, a lack of consensus about what the right age is to define a mouse as 'old' from an immunological point of view. Cohorts of mice of around 20 months of age have been repeatedly utilized in many previous studies and referred to as a representative aged cohort ${ }^{60,61}$. This map is not intended to be all encompassing or, by the nature of mass cytometry, to provide a precise enumeration of all cells. Instead, we here show mean frequencies of all identifiable immune cellular subsets in young and aged tissues.

While we have focused on three representative organs for barrier, lymphoid, and soft connective tissues, the analysis pipeline was applied to all organs presented here, and the raw data are available as an extended repository (https://zenodo.org/deposit/5593273). As a prime example of a barrier tissue, we initially focused on the lung as a reference point to validate our findings ${ }^{62}$. Of note, while we observed a relative lower frequency of $\mathrm{T}$ cells in the lungs of young mice, we also observed a relative loss of $\mathrm{T}$ helper cells with aging, which is in line with previous reports ${ }^{32}$. The most notable known changes in the myeloid compartment include a reduced abundance of tissue-resident $\mathrm{AMs}^{37}$, and $\mathrm{cDC} 1 \mathrm{~s}$, which we also saw here. While previous reports have suggested an impaired phagocytosis response in aging $\mathrm{AMs}^{37}$, the relatively higher expression of MHC-II within the total aged macrophage population that we observed suggests an interaction with $\mathrm{T}$ cells to promote tissue inflammation with age. The underlying driver of increased MHC expression may be cell intrinsic in nature and related to the progressive replacement of embryonic-derived AMs by Siglec-F Fow $M H C I I^{\text {high }} C C R 2^{\text {high }}$ monocyte-derived $\mathrm{AMs}^{63}$ occurring with aging ${ }^{64}$, or related to the increased availability of pro-inflammatory, MHC-inducing cytokines, in particular IFN- $\gamma$, in the lung microenvironment ${ }^{62}$. The loss of AMs and the resulting reduction in surfactant clearing ${ }^{36}$ has been suggested to be involved in increased mortality among older patients suffering from coronavirus 2019 (COVID-19) ${ }^{65}$. Clinical trials are currently ongoing to strengthen the AM population in severe COVID-19 infections, with stimulation of granulocyte-macrophage colony-stimulating factor, the primary growth factor for AMs (clinical trial identifiers NCT04400929 and NCT04411680) ${ }^{66}$. The current progress, as well as the risks and benefits of such treatment strategies, have recently been discussed ${ }^{67}$. We have observed certain conserved trends among the majority of aging tissues, as summarized in Fig. 5. Previous studies have alternatively reported no age-related changes in the abundance of NK cells in rodent tissues $^{68}$, or the opposite ${ }^{69}$ : this could be potentially explained by the lack of an universal overview of NK cell dynamics in a healthy aging model. Here, we consistently observed a reduction in the frequency of NK cells in tissues where they usually reside, including the lung, liver, small intestine and LNs. This general reduction in NK cells could have major implications in the aging mammal, with the overall loss of tissue protection to eliminate either virally infected or malignant cells. In line with this, we also observed a pan-loss in frequency of pDCs in the aged rodent. While this phenomenon has been described extensively in peripheral blood ${ }^{70}$, we conclude that the same pattern can be observed across the majority of aging rodent tissues.

Analysis of a further two validation cohorts of age-matched mice using spectral flow cytometry including an additional age group (4 months old) revealed that sexual maturity did not have a significant impact on the distribution of leukocytes across tissues, nor on the hematopoietic output.

In the context of healthy aging, it has been shown that in both humans and mice, CD8 $\mathrm{T}$ cell responses to new infections is decreased $^{71,72}$. Several studies have also shown the functional decline of CD8 T cells in mice, within the context of lymphoid tissues ${ }^{16,60,71,73}$, yet it is still unclear at present what the exact basis of this decline is. Through a statistically driven approach, we uncovered a tissuewide, homogeneous and significant reduction in the expression of CD103 within the naive tissue-resident CD8 T cell compartment of

Fig. 8 | Algorithm-guided analysis of hematopoietic progenitors in aging bone marrows identify LT-HSC as age-associated signature population.

a, Histogram showing the expression pattern of CellCNN-selected BM progenitor signature (left panel). Median expression has been normalized from 0 to 1. Frequency of CellCNN-selected cluster among BM progenitors (right panel). Box plot, the center is the median, lower and upper hinges depict the first and third quartiles and the whiskers stretch to 1.5 times the interquartile range from the corresponding hinge. Statistics derived from $n=10$ young and $n=10$ aged independent samples, $P=0.0232$. Statistical analysis was carried out using nonparametric Mann-Whitney-Wilcoxon tests. The $P$ value was then adjusted for multiple comparisons using a $\mathrm{BH}$ test. $P$ values of less than 0.05 were considered significant and are indicated by the numerical value on the respective graphs. $\mathbf{b}$, Violin plots ( $n=10$ young, $n=10$ aged) depicting frequency of FlowSOM-derived LT-HSC cluster among BM progenitors. Statistical analysis was carried out using nonparametric Mann-Whitney-Wilcoxon tests. $P$ values of less than 0.05 were considered significant and are indicated by the numerical value on the respective graphs. c, Median expression of CD34 within FlowSOM-derived LT-HSC cluster ( $n=10$ young, $n=10$ aged). The box plot visualizes the median, two hinges and two whiskers. The box plot represents the 25th to 75th percentiles, the whiskers are plotted following the Tukey method. All the original data points are overlaid. Statistics derived from $n=10$ young and $n=10$ aged independent samples, $P=0.0068$. Statistical analysis was carried out using nonparametric Mann-Whitney-Wilcoxon tests. $P$ values of less than 0.05 were considered significant and are indicated by the numerical value on the respective graphs. $\mathbf{d}$. Circle correlation plots showing the multiple correlation matrix between leukocyte frequencies in the lung versus BM progenitors in young and aged mice (summarized from $n=10$ young, $n=10$ aged). Results are plotted to display statistical significance $(P<0.05)$. Here, correlation coefficients $(R)$ higher than 0.6 are represented in red, and those lower than -0.6 in blue. e, Circle correlation plots showing the multiple correlation matrix between leukocyte frequencies in the sdLN versus BM progenitors in young and aged mice (summarized from $n=10$ young, $n=10$ aged). Results are plotted to display statistical significance $(P<0.05)$. Here, correlation coefficients $(R)$ higher than 0.6 are represented in red, and those lower than -0.6 in blue. 
the aging mouse (Fig. $6 \mathrm{a}-\mathrm{c}$ ). Our data revealed that the frequency of these cells did not change between the time points; rather, the cells selectively lose their CD103 expression. This phenotype can be observed across both lymphoid and non-lymphoid tissues. This particular subset did not reveal any changes in other functional (for example CD73, CD69) or activation (for example KI67, Granzyme

a CellCNN selected progenitor population

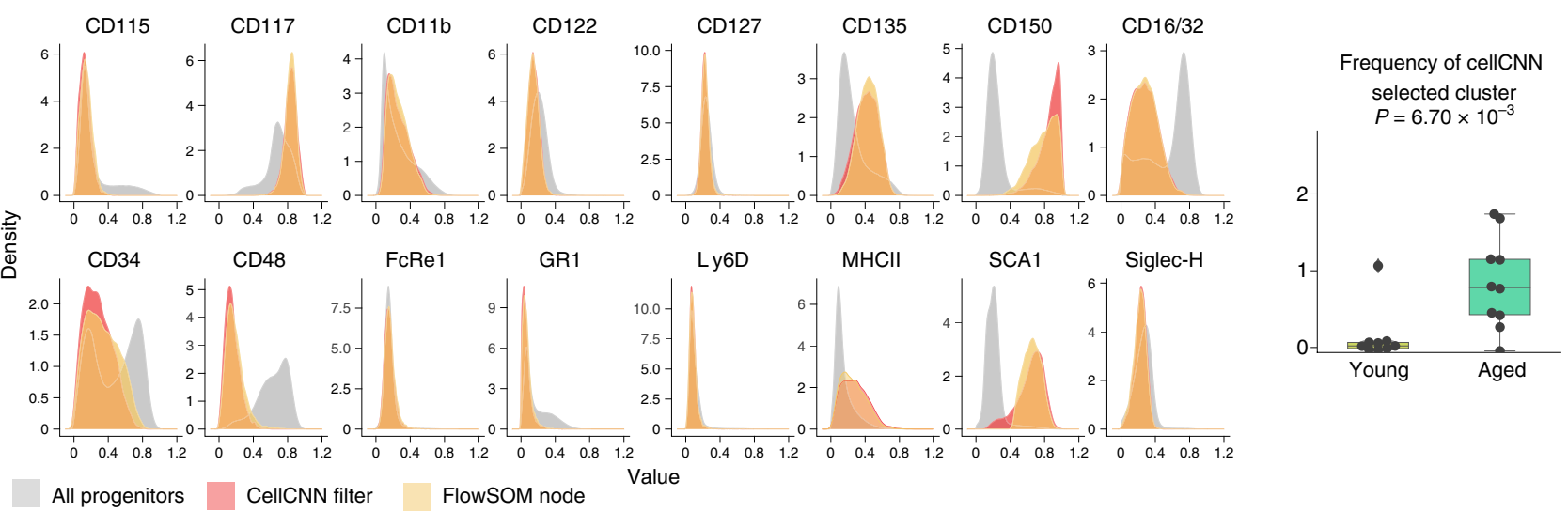

b

Frequency of matching FlowSOM cluster

c Median expression of CD34
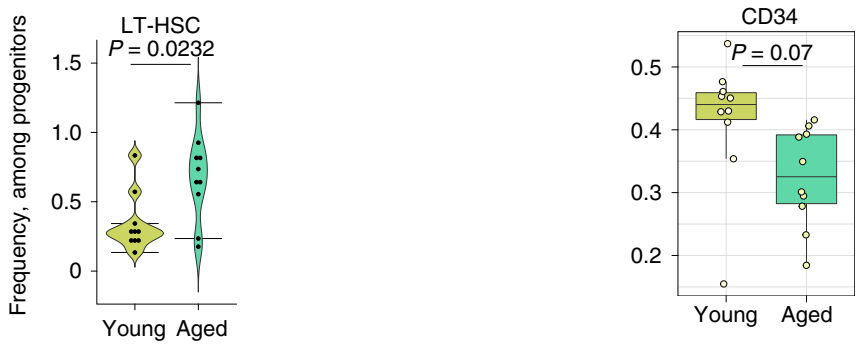

d

Circle correlation plot between lung immune cells and bone marrow progenitors
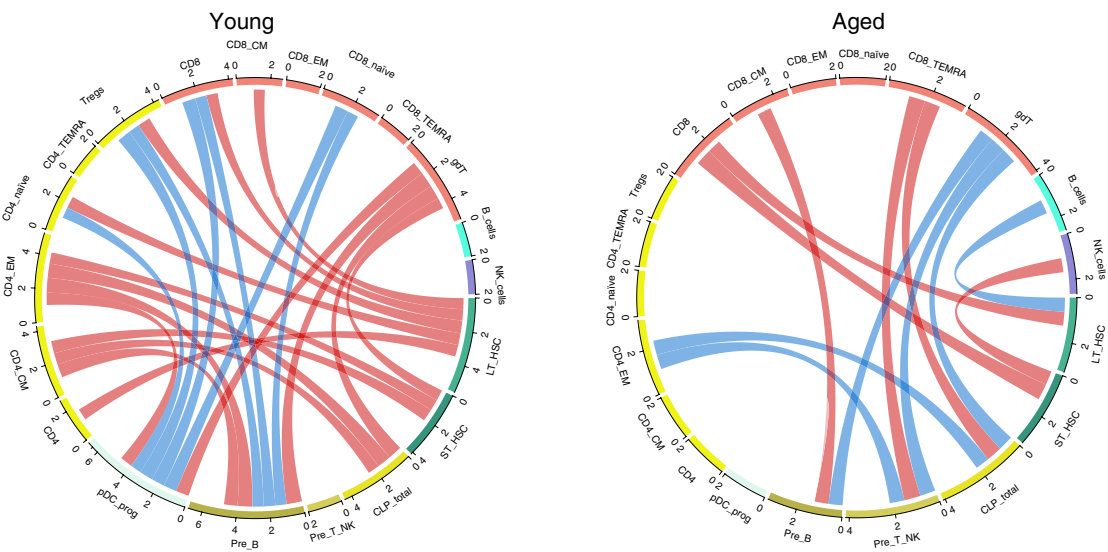

e
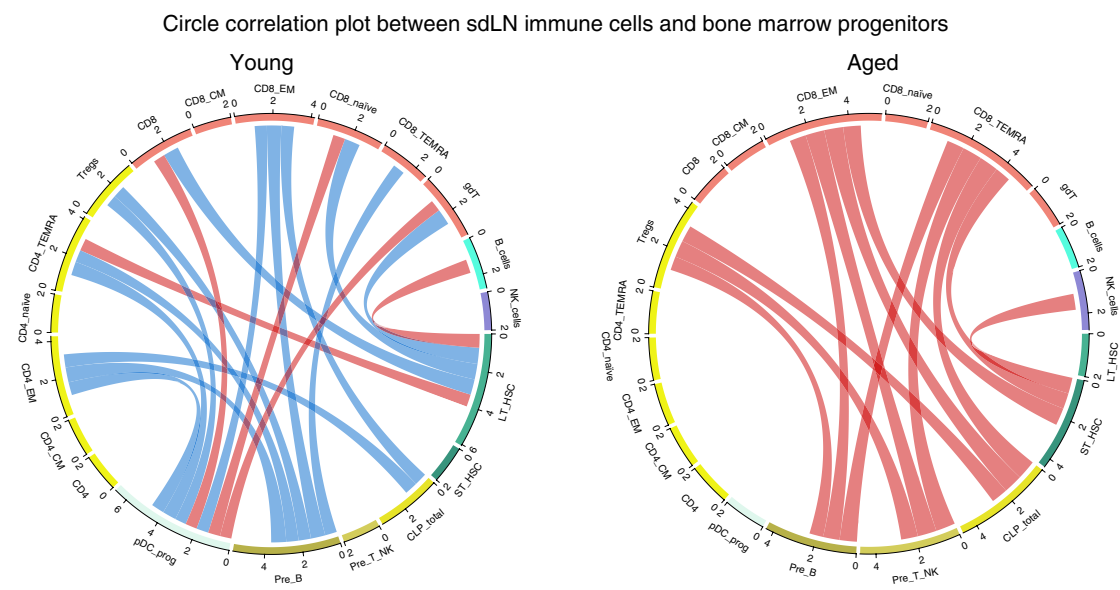
B) states. Recent studies suggest that reduced CD103 expression can be linked to increased cytotoxic function ${ }^{74,75}$.

The mechanism by which the leukocyte compartment 'adapts' to aging tissues is a matter of controversy, and again the role of inflammatory mediators remains unclear. To understand better whether certain age-related cellular patterns were caused by in situ tissue dysregulation, or predetermined at the stem cell level, we interrogated the hematopoietic precursor compartment using spectral flow cytometry. The widespread loss of NK cells and CD4 T cells in the aging mouse correlates with significantly reduced Ly6 $\mathrm{D}^{\text {low }}$ CLPs in the aging bone marrow. Finally, the reduced frequency and numbers of pDC-committed CLP fraction further complement the general trends we described in our aging immune atlas. Overall, we confirmed a skewing of the hematopoietic compartment towards myelopoiesis ${ }^{76}$, and a systemic impairment of adaptive immune compartment (underpinned by the reduction of CLPs) accounting for the hampered antiviral and antitumor responses associated with aging. Unbiased, algorithm-driven analyses identified a subset of hematopoietic precursors, namely LT-HSC, to expand during aging. These aged LT-HSCs lack CD34 expression, a phenotype associated with reduced differentiation potential towards lymphoid cells $s^{52,53}$. Our findings suggest in fact that the 'aging phenotype' observed in the immune compartment of aging tissues is not primarily the result of a cell-intrinsic, programmed adaptation or that the aging tissue would firmly dictate its leukocyte niche. Instead, our data support the notion that immune aging is largely the result of hematopoietic output.

In conclusion, here we introduce a comprehensive leukocyte atlas of the aging immune system, with a focus on the relative abundance of all leukocyte lineages within 12 organs. This dataset is presented as a resource, to facilitate future age-related studies with more focus on tissue-specific and tissue-resident leukocyte subsets in the naive mouse model. We present this resource to the scientific community in the hope of establishing a baseline, naive comprehensive dataset to build on for future investigative work in the C57/ BL6 aging mouse model. We hope to have demonstrated its utility in drawing important conclusions about the state of the overall and tissue-specific immune systems across the murine model.

\section{Methods}

In vivo experimental details. Mice used for all experiments were $\mathrm{C} 57 \mathrm{BL} / 6 \mathrm{JRj}$, wild-type mice acquired from Janvier laboratories, where the animals were housed in the same housing facility from birth until purchase. Then, the animals were acclimatized, together, at the animal housing facility of the University of Zurich for at least 2 weeks before experimental procedures. Mice were kept in a 12-hour lightdark cycle, with an ambient temperature range within $20-24^{\circ} \mathrm{C}$; humidity ranged from $30 \%$ to $70 \%$.

Male and female mice from the 'young' group were aged approximately 7-8 weeks, 'middle' were 16 weeks and 'aged' were between 18 and 20 months.

For our initial analysis cohort, we devised four experimental groups: $n=4$ young adult male, $n=4$ young adult female; $n=4$ aged female; and $n=3$ aged male C57/BL6 mice.

For our validation cohort 1 , we devised the following experimental group: $n=5$ young adult male, $n=5$ young adult female; $n=5$ aged female; and $n=5$ aged male C57/BL6 mice.

For our validation cohort 2 , we procured: $n=5$ juvenile male, $\mathrm{n}=5$ young adult male, $n=5$ juvenile female, $n=5$ young adult female; $n=5$ aged female; and $n=5$ aged male C57/BL6 mice.

All animal experiments performed in this study were approved by and in adherence with the Cantonal Veterinary Office Zurich.

Tissue harvesting, and single-cell suspension preparation. Tissues from mice (lung, liver, visceral adipose tissue, brown adipose tissue, small intestine, thymus, testes, MesLNs, sdLNs, spleen, blood and BM) were harvested as described previously ${ }^{4}$. Briefly, mice were killed by intra-peritoneal (i.p.) injection of pentobarbital $\left(50 \mu \mathrm{l}\right.$; at $\left.300 \mathrm{mg} \mathrm{ml}^{-1}\right)$ followed by trans-cardiac perfusion with phosphate buffered saline (PBS) and heparin $\left(5 \mathrm{Units}^{-1}\right)$. Each organ was harvested and cut into small pieces, using scissors, inside an Eppendorf tube. Next, the samples were incubated with digestion medium (HBSS + + with $2 \%$ fetal calf serum (FCS), $2 \mathrm{mM}$ HEPES, DNAse $(50 \mu \mathrm{l}$ per $10 \mathrm{ml})$ and Collagenase IV) at $37^{\circ} \mathrm{C}$ while shaking. The exact medium concentrations and incubation times for each organ are described in Supplementary Table 2.
Enzymatic digestion was stopped with ethylenediamine tetraacetic acid (EDTA) $5 \mathrm{mM}$. The samples were homogenized using a syringe and a $24 \mathrm{G}$ needle, and the homogenate was filtered through a $100 \mu \mathrm{M}$ cell strainer. SLTs were subjected to Red Blood Cell lysis (Biolegend) ( $5 \mathrm{~min}$ at room temperature). The CNS cell suspensions were further enriched by Percoll gradient $(30 \%)$ centrifugation $\left(1,590 \mathrm{~g}, 4^{\circ} \mathrm{C}, 30 \mathrm{~min} \text {, no brake }\right)^{77}$ and thymus samples too (centrifugation at 2,750 r.p.m., $4^{\circ} \mathrm{C}, 30 \mathrm{~min}$, no brake). Spleen samples from the initial cohort were subjected to myeloid cell enrichment using negative bead selection (MACS MicroBeads and Column, Miltenyi Biotec) for ter119, CD3, CD19 and CD5. The samples were then ready for labeling with antibodies for mass or fluorescence cytometry.

Mass-cytometry analysis. Antibodies used were either acquired from Fluidigm or conjugated in-house using antibody-labeling kits purchased from Fluidigm. Following antibody conjugation, reagents were titrated and tested as part of the final panel accordingly. As described previously ${ }^{78}$, CD45 antibodies conjugated to six palladium metal isotopes (104 Pd, $105 \mathrm{Pd}, 106 \mathrm{Pd}, 108 \mathrm{Pd}$ and $110 \mathrm{Pd}$; all from Trace Sciences International) were used for live immune cell barcoding. All individual tissue samples were labeled with a combination of three different barcoding antibodies for $30 \mathrm{~min}$ at $37^{\circ} \mathrm{C}$ on a rotary shaker. Then, following two wash steps (with PBS $+2 \%$ FCS), all samples from the same organ were combined together. Combined samples were labeled with the primary antibodies for $30 \mathrm{~min}$ at $37^{\circ} \mathrm{C}$, then, following a wash step, the cells were labeled with the secondary antibodies for $20 \mathrm{~min}$ at $4^{\circ} \mathrm{C}$. To identify dead cells, $2.5 \mu \mathrm{M}$ cisplatin (Sigma-Aldrich) was added for $2 \mathrm{~min}$ on ice. After washing, samples were fixed with $1.6 \%$ paraformaldehyde (PFA; Electron Microscopy Sciences) in PBS for up to 4 days at $4{ }^{\circ} \mathrm{C}$. On the day of acquisition, samples were incubated with intercalating solution (Iridium (Sigma) in MaxPar Fix/Perm buffer (Fluidigm)) for $1-3 \mathrm{~h}$ ) at $4^{\circ} \mathrm{C}$. Shortly before acquisition, the samples were washed twice with FACS buffer and once with Maxpar water (Fluidigm). Barcoded samples were acquired on a Helios mass cytometer (Fluidigm), using CyTOF Software v.6.7. Quality control and tuning processes on the Helios were performed on a daily basis, as well as before and during acquisition as required. Data from different days and across acquisition time were normalized by adding five element beads to the sample immediately before acquisition and using the MATLAB-based normalization software, as described previously ${ }^{79}$.

For mass cytometry analysis, we used the antibodies listed in Supplementary Table 3 .

Flow-cytometry analysis. All reagents were tested and titrated before use in experiments. To discern live cells, all individual samples were incubated with LIVE/DEAD fixable blue dead cell kit (Thermofisher) in PBS, at a concentration of $1: 800$, for $30 \mathrm{~min}$ on ice. Following a wash step, the cells were then incubated with CD16/32 antibody at a concentration of 1:400 (the purified form (Thermofisher) for lymphoid panel samples, and CD16/32 BV605 for myeloid panel samples) in PBS for $20 \mathrm{~min}$ on ice.

All subsequent antibody incubations were carried out in PBS supplemented with $2 \%$ FCS and 20\% Brilliant Stain Buffer (BD Biosciences). Cells were incubated with the primary antibody cocktail for $30 \mathrm{~min}$ at $4{ }^{\circ} \mathrm{C}$. Following another wash step, the cells were incubated in the secondary antibody cocktail for $20 \mathrm{~min}$ at $4^{\circ} \mathrm{C}$.

When intracellular labeling was required, cells were permeabilized using Foxp3/Transcription Factor Staining Buffer Set (eBioscience) according to the manufacturer's instructions, for $45 \mathrm{~min}$ at $4^{\circ} \mathrm{C}$. Subsequently, the sample was washed once in Perm/Wash buffer (eBioscience) and incubated in the antibody mixture in Perm/Wash buffer overnight at $4^{\circ} \mathrm{C}$. The samples were washed once in Perm/Wash buffer (eBioscience) and centrifuged to pellet the cells before resuspension in PBS before flow-cytometry data acquisition. Samples were acquired on an Aurora spectral analyzer (Cytek Biosciences) using SpectroFlo Software (v.2.0) following daily quality control procedures as instructed by the manufacturer.

For spectral flow cytometry of the lymphoid and myeloid compartments of 11 organs, we used the antibodies described in Supplementary Table 4.

For spectral flow cytometry of the hematopoietic compartment in the bone marrow, we used the antibodies described in Supplementary Table 5.

High-dimensional data preprocessing and statistical analysis. From the raw data acquired by mass cytometry, using FlowJo software v.10.6.2 and 10.7.1 (TreeStar), live cells were identified using manual gating on event length, DNA (191Ir and 193Ir) and live cells (195Pt). Debarcoding of each sample was achieved using Boolean gating. For flow-cytometry data, the compensation matrix was corrected in FlowJo (TreeStar) by pregating on live immune cells, and the total fraction of live, singlet immune cells was exported. Data were then transformed with an inverse hyperbolic sine ( $\operatorname{arcsinh}$ ) function (cofactors ranging between 5 and 18,000 ) then imported into the R environment (v.3.6.1) for subsequent analysis ${ }^{80}$.

The high-dimensional analysis was carried out using the $\mathrm{R}$ environment, based on the workflow described previously by Hartmann et al. ${ }^{20}$. Briefly, UMAPs were generated using the package umap v.0.2.7.0 (ref. ${ }^{21}$ ), and FlowSOM clustering was overlaid on the dimensionality reduction maps ${ }^{19}$. Frequency plots were generated using the ggplot2 package v.3.3.5, and heatmaps were generated using the pheatmap package v.1.0.12 
Force-directed layouts were generated by sampling 1,500 cells from each mouse sample, and using the ForceAtlas 2 algorithm ${ }^{48}$ integrated in the VorteX graphical clustering environment creating unweighted edges between the nodes based on the ten nearest neighbors ${ }^{81}$. Resulting graphs were further modified using the Gephi Toolkit v.0.9.2.

Scaffold networks were computed using the improved version of the initial Scaffold package consisting of grappolo, vite and panorama ${ }^{82}$. For the construction of lymphocyte and myeloid cell networks, resulting graphs were rearranged in the igraph framework using the graphopt algorithm (as described in http://www. schmuhl.org/graphopt/).

CellCNN-based analysis was performed by training a randomly selected subset of cells. Default values were used for all CellCNN hyperparameters ${ }^{51}$. Default values were used for most CellCNN hyperparameters, except for the following: ncell equals 3,000;-no_arcsinh;_no_scale;-filter_response_thres 0.3 ; - train_ perc 0.5 . multidimensional scaling plots were generated using the stats package v.4.2.0 and displayed using ggscatter v.0.4.0. Correlation matrices were calculated using Hmisc v.4.5-0 and generated using circlize v.0.4.13 in conjunction with corrplot v. 0.90. All other plots were generated using ggplot2.

Statistical analysis was carried out using nonparametric Mann-WhitneyWilcoxon tests. The $P$ value was then adjusted for multiple comparisons using a Benjamini-Hochberg $(\mathrm{BH})$ test $^{83}$ using the R package rstatix v.0.7.0. $P$ values of less than 0.05 were considered significant and are indicated by an asterisk $\left(^{*}\right)$ or the numerical value on the respective graphs.

Reporting Summary. Further information on research design is available in the Nature Research Reporting Summary linked to this article.

\section{Data availability}

FCS files of the mass and spectral flow-cytometry data as well as extended data relating to cell counts are available for download through the following repository link: https://zenodo.org/deposit/5593273.

\section{Code availability}

An R-based script for data analysis is available through the following repository link: https://zenodo.org/deposit/5593273.

Received: 22 December 2020; Accepted: 4 November 2021; Published online: 16 December 2021

\section{References}

1. Pawelec, G. \& Solana, R. Immunosenescence. Immunol. Today 18, 514-516 (1997).

2. Franceschi, C. et al. Inflamm-aging: an evolutionary perspective on immunosenescence. Ann. N. Y. Acad. Sci. 908, 244-254 (2000).

3. Adlung, L., Amit, I. \& Elinav, E. Embrace the fat when getting old. Aging 11, 8730-8732 (2019).

4. Mrdjen, D. et al. High-dimensional single-cell mapping of central nervous system immune cells reveals distinct myeloid subsets in health, aging, and disease. Immunity 48, 380-395.e6 (2018).

5. Reichert, T., Chowell, G. \& McCullers, J. A. The age distribution of mortality due to influenza: pandemic and peri-pandemic. BMC Med. 10, 162 (2012).

6. Clayton, D. \& Schifflers, E. Models for temporal variation in cancer rates. II: Age-period-cohort models. Stat. Med. 6, 469-481 (1987).

7. Ridker, P. M., Rifai, N., Stampfer, M. J. \& Hennekens, C. H. Plasma concentration of interleukin- 6 and the risk of future myocardial infarction among apparently healthy men. Circulation 101, 1767-1772 (2000).

8. Brunet, A. \& Berger, S. L. Epigenetics of aging and aging-related disease. Journals Gerontol. Ser. A Biomed. Sci. Med. Sci 69, S17-S20 (2014).

9. Gustafson, C. E., Kim, C., Weyand, C. M. \& Goronzy, J. J. Influence of immune aging on vaccine responses. J. Allergy Clin. Immunol. 145, 1309-1321 (2020).

10. Nikolich-Žugich, J. The twilight of immunity: emerging concepts in aging of the immune system. Nat. Immunol. 19, 10 (2018).

11. Alam, M. S. et al. Oral exposure to Listeria monocytogenes in aged IL-17RKO mice: apossible murine model to study listeriosis in susceptible populations. Microb. Pathog. 99, 236-246 (2016).

12. Mekker, A. et al. Immune senescence: relative contributions of age and cytomegalovirus infection. PLoS Pathog. 8, e1002850 (2012).

13. Lin, Y. et al. Changes in blood lymphocyte numbers with age in vivo and their association with the levels of cytokines/cytokine receptors. Immun. Ageing 13, 24 (2016)

14. Wertheimer, A. M. et al. Aging and cytomegalovirus infection differentially and jointly affect distinct circulating $\mathrm{T}$ cell subsets in humans. J. Immunol. 192, 2143-2155 (2014)

15. Deshpande, N. R., Parrish, H. L. \& Kuhns, M. S. Self-recognition drives the preferential accumulation of promiscuous CD4(+) T-cells in aged mice. eLife 4, e05949 (2015)
16. Decman, V. et al. Defective CD8 T cell responses in aged mice are due to quantitative and qualitative changes in virus-specific precursors. J. Immunol. 188, 1933-1941 (2012).

17. Montgomery, R. R. \& Shaw, A. C. Paradoxical changes in innate immunity in aging: recent progress and new directions. J. Leukoc. Biol. 98, 937-943 (2015)

18. Schulte, H., Mühlfeld, C. \& Brandenberger, C. Age-related structural and functional changes in the mouse lung. Front. Physiol. 10, 1466 (2019).

19. Van Gassen, S. et al. FlowSOM: Using self-organizing maps for visualization and interpretation of cytometry data. Cytom. Part A 87, 636-645 (2015).

20. Hartmann, F. J. et al. High-dimensional single-cell analysis reveals the immune signature of narcolepsy. J. Exp. Med. https://doi.org/10.1084/ jem.20160897 (2016).

21. McInnes, et al. UMAP: Uniform Manifold Approximation and Projection. J. Open Source Softw. 3, 861 (2018).

22. Junker, F., Gordon, J. \& Qureshi, O. Fc gamma receptors and their role in antigen uptake, presentation, and $\mathrm{T}$ cell activation. Front. Immunol. 11, 1393 (2020)

23. Sheng, J. et al. A discrete subset of monocyte-derived cells among typical conventional type 2 dendritic cells can efficiently cross-present. Cell Rep. 21, 1203-1214 (2017).

24. Balan, S., Saxena, M. \& Bhardwaj, N. in International Review of Cell and Molecular Biology (eds Galluzzi, L. \& Lhuillier, C.) Vol. 348, 1-68 (Elsevier Inc., 2019).

25. Dress, R. J. et al. Plasmacytoid dendritic cells develop from Ly6D+ lymphoid progenitors distinct from the myeloid lineage. Nat. Immunol. 20, 852-864 (2019)

26. Buja, A. et al. Data visualization with multidimensional scaling. J. Comput. Graph. Stat. 17, 444-472 (2012).

27. M., N. et al. CyTOF workflow: differential discovery in high-throughput high-dimensional cytometry datasets. F1000Research 6, 748 (2017).

28. Lu, R. J. et al. Multi-omic profiling of primary mouse neutrophils predicts a pattern of sex and age-related functional regulation. Nat. Aging 1, 715-733 (2021).

29. Zaynagetdinov, R. et al. Identification of myeloid cell subsets in murine lungs using flow cytometry. Am. J. Respir. Cell Mol. Biol. 49, 180-189 (2013).

30. Ji, J. J. \& Fan, J. Discovering myeloid cell heterogeneity in the lung by means of next generation sequencing. Military Med. Res. 6, 33 (2019).

31. Meiners, S., Eickelberg, O. \& Königshoff, M. Hallmarks of the ageing lung. Eur. Respir. J. 45, 807-827 (2015).

32. Toapanta, F. R. \& Ross, T. M. Impaired immune responses in the lungs of aged mice following influenza infection. Respir. Res. https://doi. org/10.1186/1465-9921-10-112 (2009).

33. Wyburn, K. R., Jose, M. D., Wu, H., Atkins, R. C. \& Chadban, S. J. The role of macrophages in allograft rejection. Transplantation $\mathbf{8 0}$, 1641-1647 (2005)

34. Cho, K. W. et al. An MHC II-dependent activation loop between adipose tissue macrophages and CD4+ T cells controls obesity-induced inflammation. Cell Rep. 9, 605-617 (2014).

35. Fulton, S. A. et al. Inhibition of major histocompatibility complex II expression and antigen processing in murine alveolar macrophages by Mycobacterium bovis BCG and the 19-kilodalton mycobacterial lipoprotein. Infect. Immun. 72, 2101-2110 (2004).

36. Yao, Y. et al. Induction of autonomous memory alveolar macrophages requires $\mathrm{T}$ cell help and is critical to trained immunity. Cell 175, 1634-1650. e17 (2018).

37. Wong, C. K. et al. Aging impairs alveolar macrophage phagocytosis and increases influenza-induced mortality in Mice. J. Immunol. 199, 1060-1068 (2017)

38. Della Bella, S. et al. Peripheral blood dendritic cells and monocytes are differently regulated in the elderly. Clin. Immunol. 122, 220-228 (2007).

39. Turner, V. M. \& Mabbott, N. A. Structural and functional changes to lymph nodes in ageing mice. Immunology 151, 239-247 (2017).

40. Hill, D. A. et al. Distinct macrophage populations direct inflammatory versus physiological changes in adipose tissue. Proc. Natl Acad. Sci. USA 115, E5096-E5105 (2018)

41. Dye, L., Boyle, N. B., Champ, C. \& Lawton, C. The relationship between obesity and cognitive health and decline. Proc. Nutr. Soc. 76 , 443-454 (2017)

42. van Splunter, M. et al. Plasmacytoid dendritic cell and myeloid dendritic cell function in ageing: a comparison between elderly and young adult women. PLoS ONE14, e0225825 (2019)

43. Cohen, J. in Statistical Power Analysis for the Behavioral Sciences xiii-xv (Elsevier, 1977); https://doi.org/10.1016/B978-0-12-179060-8.50005-0 44. Cohen, J. A power primer. Psychol. Bull. 112, 155-159 (1992).

45. Min, H., Montecino-Rodriguez, E. \& Dorshkind, K. Effects of aging on the common lymphoid progenitor to pro-B cell transition. J. Immunol. 176, 1007-1012 (2006).

46. Rossi, D. J. et al. Cell intrinsic alterations underlie hematopoietic stem cell aging. Proc. Natl Acad. Sci. USA 102, 9194-9199 (2005). 
47. Grover, A. et al. Single-cell RNA sequencing reveals molecular and functional platelet bias of aged haematopoietic stem cells. Nat. Commun. 7, 11075-11075 (2016)

48. Jacomy, M., Venturini, T., Heymann, S. \& Bastian, M. ForceAtlas2, a continuous graph layout algorithm for handy network visualization designed for the Gephi software. PLoS ONE https://doi.org/10.1371/journal. pone.0098679 (2014).

49. Kwok, I. et al. Combinatorial single-cell analyses of granulocyte-monocyte progenitor heterogeneity reveals an early uni-potent neutrophil progenitor. J. Clean. Prod. 53, 303-318.e5 (2020).

50. Rodrigues, P. F. et al. Distinct progenitor lineages contribute to the heterogeneity of plasmacytoid dendritic cells. Nat. Immunol. 19, 711-722 (2018).

51. Arvaniti, E. \& Claassen, M. Sensitive detection of rare disease-associated cell subsets via representation learning. Nat. Commun. 8, 14825 (2017).

52. Rossi, D. J. et al. Cell intrinsic alterations underlie hematopoietic stem cell aging. Proc. Natl Acad. Sci. USA 102, 9194-9199 (2005).

53. Sudo, K., Ema, H., Morita, Y. \& Nakauchi, H. Age-associated characteristics of murine hematopoietic stern cells. J. Exp. Med. 192, 1273-1280 (2000).

54. Prather, A. A. et al. Associations between chronic caregiving stress and $\mathrm{T}$ cell markers implicated in immunosenescence. Brain. Behav. Immun. 73, 546-549 (2018).

55. Akbar, A. N., Henson, S. M. \& Lanna, A. Senescence of T lymphocytes: implications for enhancing human immunity. Trends Immunol. 37, 866-876 (2016).

56. Szabo, P. A. et al. Single-cell transcriptomics of human T cells reveals tissue and activation signatures in health and disease. Nat Commun. 10, 4706 (2019).

57. Schaum, N. et al. Single-cell transcriptomics of 20 mouse organs creates a Tabula muris. Nature 562, 367-372 (2018).

58. Almanzar, N. et al. A single-cell transcriptomic atlas characterizes ageing tissues in the mouse. Nature 583, 590-595 (2020).

59. Johnson, M. Laboratory mice and rats. Mater. Methods 2, (2012).

60. Quinn, K. M. et al. Age-related decline in primary $\mathrm{CD}^{+} \mathrm{T}$ cell responses is associated with the development of senescence in virtual memory CD8 ${ }^{+} \mathrm{T}$ cells. Cell Rep. 23, 3512-3524 (2018).

61. Leins, H. et al. Aged murine hematopoietic stem cells drive aging-associated immune remodeling. Blood 132, 565-576 (2018).

62. Angelidis, I. et al. An atlas of the aging lung mapped by single cell transcriptomics and deep tissue proteomics. Nat Commun. 10, 963 (2019).

63. Machiels, B. et al. A gammaherpesvirus provides protection against allergic asthma by inducing the replacement of resident alveolar macrophages with regulatory monocytes. Nat. Immunol. 18, 1310-1320 (2017).

64. Liu, Z. et al. Fate mapping via Ms4a3-expression history traces monocytederived cells. Cell 178, 1509-1525.e19 (2019).

65. Zviran, A. et al. Genome-wide cell-free DNA mutational integration enables ultra-sensitive cancer monitoring. Nat. Med. 26, 1114-1124 (2020).

66. Bosteels, C. et al. Sargramostim to treat patients with acute hypoxic respiratory failure due to COVID-19 (SARPAC): a structured summary of a study protocol for a randomised controlled trial. Trials 21, 491 (2020).

67. Lang, F. M., Lee, K. M. C., Teijaro, J. R., Becher, B. \& Hamilton, J. A. GM-CSF-based treatments in COVID-19: reconciling opposing therapeutic approaches. Nat. Rev. Immunol. 20, 507-514 (2020).

68. Nogusa, S., Ritz, B. W., Kassim, S. H., Jennings, S. R. \& Gardner, E. M. Characterization of age-related changes in natural killer cells during primary influenza infection in mice. Mech. Ageing Dev. 129, 223-230 (2008).

69. Beli, E. et al. Natural killer cell development and maturation in aged mice. Mech. Ageing Dev. 135, 33-40 (2014).

70. Jing, Y. et al. Aging is associated with a numerical and functional decline in plasmacytoid dendritic cells, whereas myeloid dendritic cells are relatively unaltered in human peripheral blood. Hum. Immunol. 70, 777-784 (2009)

71. Briceño, O. et al. Reduced naïve CD8+ T-cell priming efficacy in elderly adults. Aging Cell 15, 14-21 (2016).

72. Jiang, J., Fisher, E. M. \& Murasko, D. M. Intrinsic defects in CD8 T cells with aging contribute to impaired primary antiviral responses. Exp. Gerontol. 48 , 579-586 (2013).

73. Renkema, K. R., Li, G., Wu, A., Smithey, M. J. \& Nikolich-Žugich, J. Two separate defects affecting true naive or virtual memory $\mathrm{T}$ cell precursors combine to reduce naive $\mathrm{T}$ cell responses with aging. J. Immunol. 192, 151-159 (2014).
74. Lardy, M. et al. T Cells + regulatory CD8 CD103 is a marker for alloantigeninduced. J. Immunol. https://doi.org/10.4049/jimmunol.177.5.2775 (2021).

75. Uss, E. et al. CD103 is a marker for alloantigen-induced regulatory CD $8+\mathrm{T}$ cells. J. Immunol. 177, 2775-2783 (2006).

76. Yamamoto, R. et al. Large-scale clonal analysis resolves aging of the mouse hematopoietic stem cell compartment. Cell Stem Cell 22, 600-607.e4 (2018).

77. Mundt, S. et al. Conventional DCs sample and present myelin antigens in the healthy CNS and allow parenchymal T cell entry to initiate neuroinflammation. Sci. Immunol 4, 8380 (2019).

78. Mei, H. E., Leipold, M. D., Schulz, A. R., Chester, C. \& Maecker, H. T. Barcoding of live human peripheral blood mononuclear cells for multiplexed mass cytometry. J. Immunol. 194, 2022-2031 (2015).

79. Finck, R. et al. Normalization of mass cytometry data with bead standards. Cytom. Part A 83A, 483-494 (2013).

80. Bendall, S. C. et al. Single-cell mass cytometry of differential immune and drug responses across a human hematopoietic continuum. Science (80) 332, 687-696 (2011).

81. Samusik, N., Good, Z., Spitzer, M. H., Davis, K. L. \& Nolan, G. P. Automated mapping of phenotype space with single-cell data. Nat. Methods https://doi. org/10.1038/nmeth.3863 (2016).

82. Spitzer, M. H. et al. An interactive reference framework for modeling a dynamic immune system. Science (80). 349, 1259425 (2015).

83. Benjamini, Y. \& Hochberg, Y. Controlling the false discovery rate: a practical and powerful approach to multiple testing. J. R. Stat. Soc. Ser. B https://doi. org/10.1111/j.2517-6161.1995.tb02031.x (1995).

\section{Acknowledgements}

We thank L. Ducimetière, E. Galli, J. Gschwend, J. Komuczki, I. Lelios, A. Rita Liuzzi, W. Mildenberger and S. Tuzlak for experimental support during the harvest and processing of various mouse tissues for mass-cytometry experiments; the Cytometry Facility (University of Zurich) for technical assistance; and the Bodenmiller lab (University of Zurich) for sharing access to their CyTOF facility during a technical issue. We thank L. Robinson from Insight Editing London for critical review and editing of the manuscript. Cartoon images used in Fig. 1, depicting mice, internal organs, containers and cells were created with and adapted from smart.servier.com. This work was funded by grants from the Swiss National Science Foundation (grant number 310030_188450) and European Research Council AdG (IMPACT, 882424), both awarded to B.B.

\section{Author contributions}

S.K. conceptualized, designed and performed all the cytometry experiments, as well as the analysis of the data. F.I. and E.F. helped with all cytometry experiments and data analysis. Both F.I. and E.F. have the right to list their name first in their CV. D.C., A.A., M.L., S.M. and F.R. provided technical support with both mass and spectral flow-cytometry experiments. M.A., D.B., G.L., M.M., C.S., S.S., C.U., S.U., M.V. and P.Z. provided technical support with spectral flow-cytometry experiments. M.G. and S.T. provided intellectual input. D.D.F. analyzed the hematopoiesis datasets and helped review the manuscript. S.K. and B.B. wrote the manuscript. B.B. supervised and funded the study.

\section{Competing interests}

The authors declare no competing interests.

\section{Additional information}

Extended data is available for this paper at https://doi.org/10.1038/s43587-021-00148-x. Supplementary information The online version contains supplementary material available at https://doi.org/10.1038/s43587-021-00148-x.

Correspondence and requests for materials should be addressed to Burkhard Becher.

Peer review information Nature Aging and the authors of this article thank the anonymous reviewers for their contribution to the peer review of this work.

Reprints and permissions information is available at www.nature.com/reprints.

Publisher's note Springer Nature remains neutral with regard to jurisdictional claims in published maps and institutional affiliations.

(c) The Author(s), under exclusive licence to Springer Nature America, Inc. 2021 
Experimental statistics

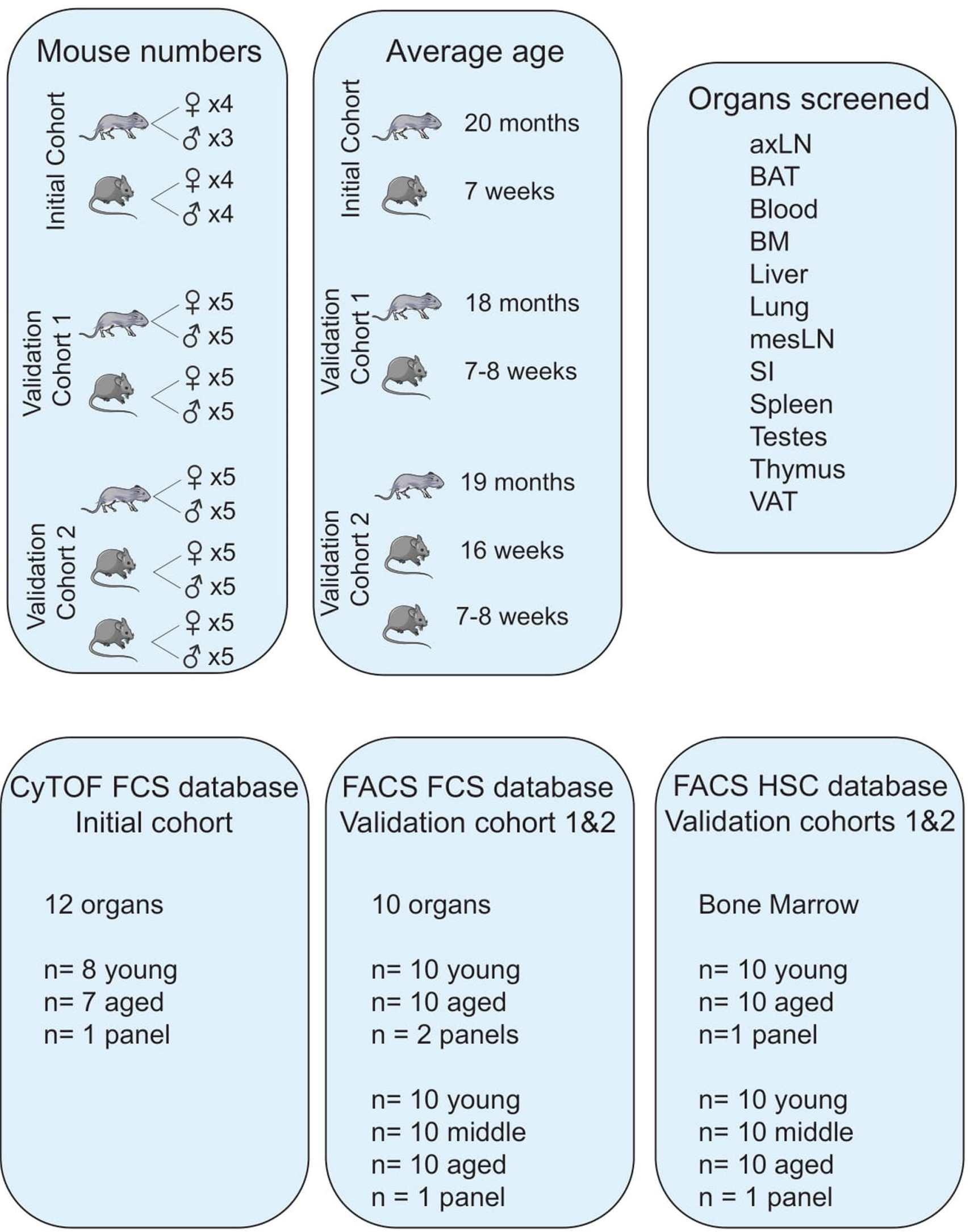

Extended Data Fig. 1 | Summary of experimental groups related to Fig. 1. Summary of experimental groups. 
A MDS plot of lymphoid compartment, examplary organ sdLN
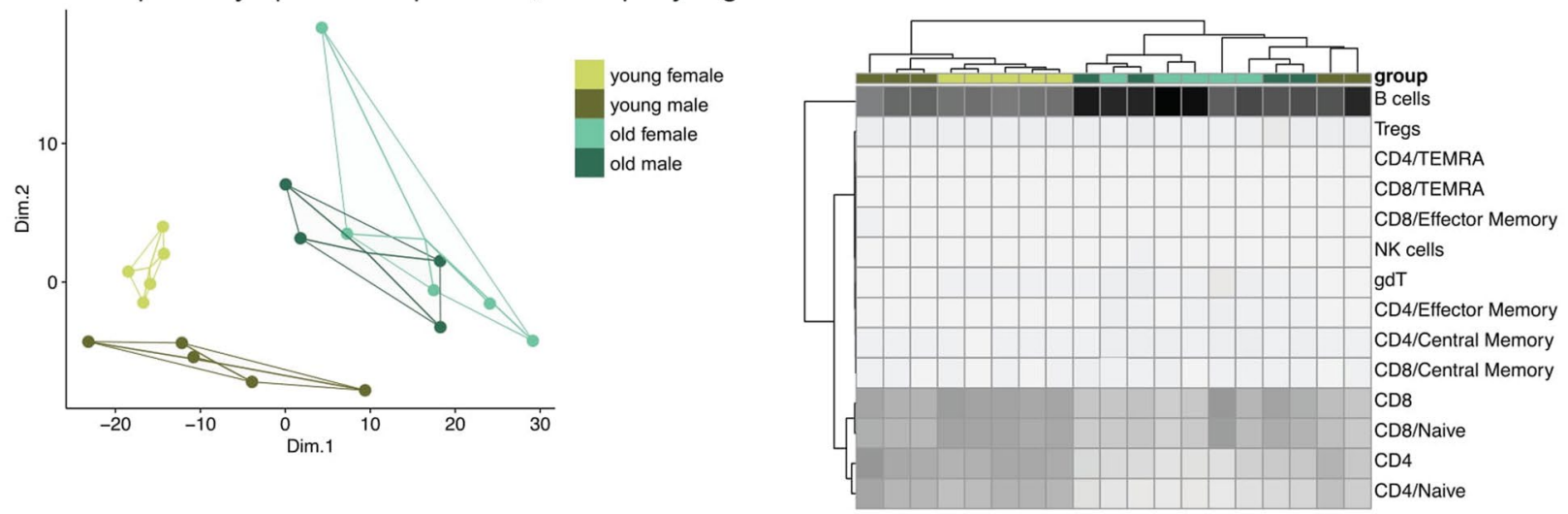

B MDS plot of myeloid compartment, examplary organ sdLN
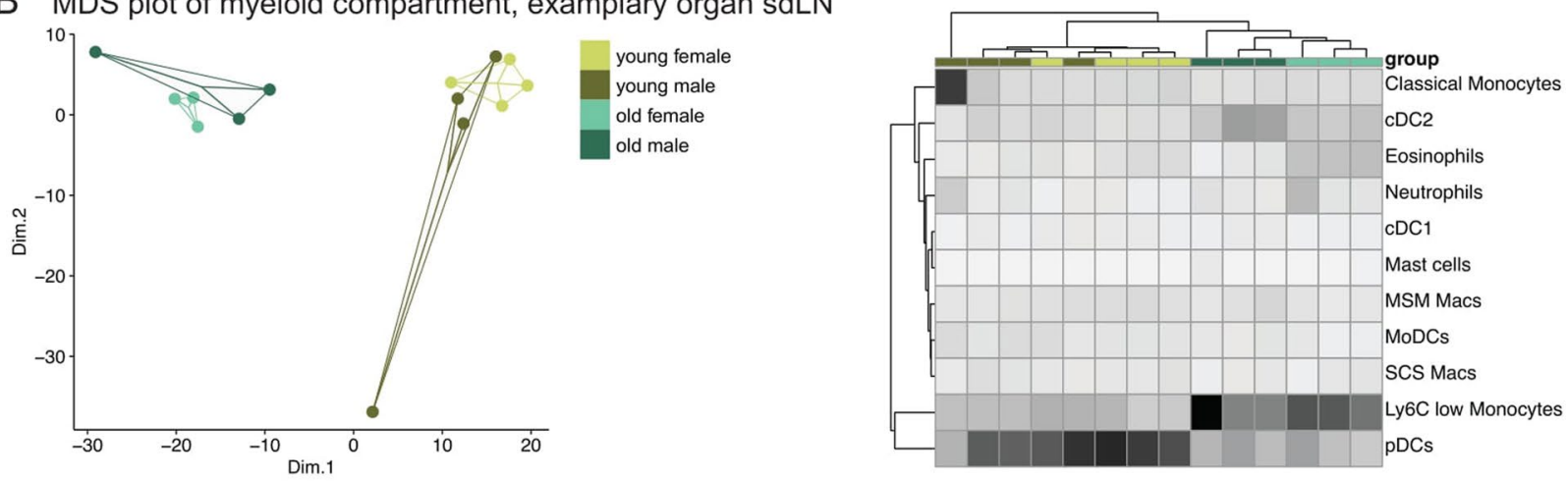

C MDS plot of progenitor compartment within bone marrow
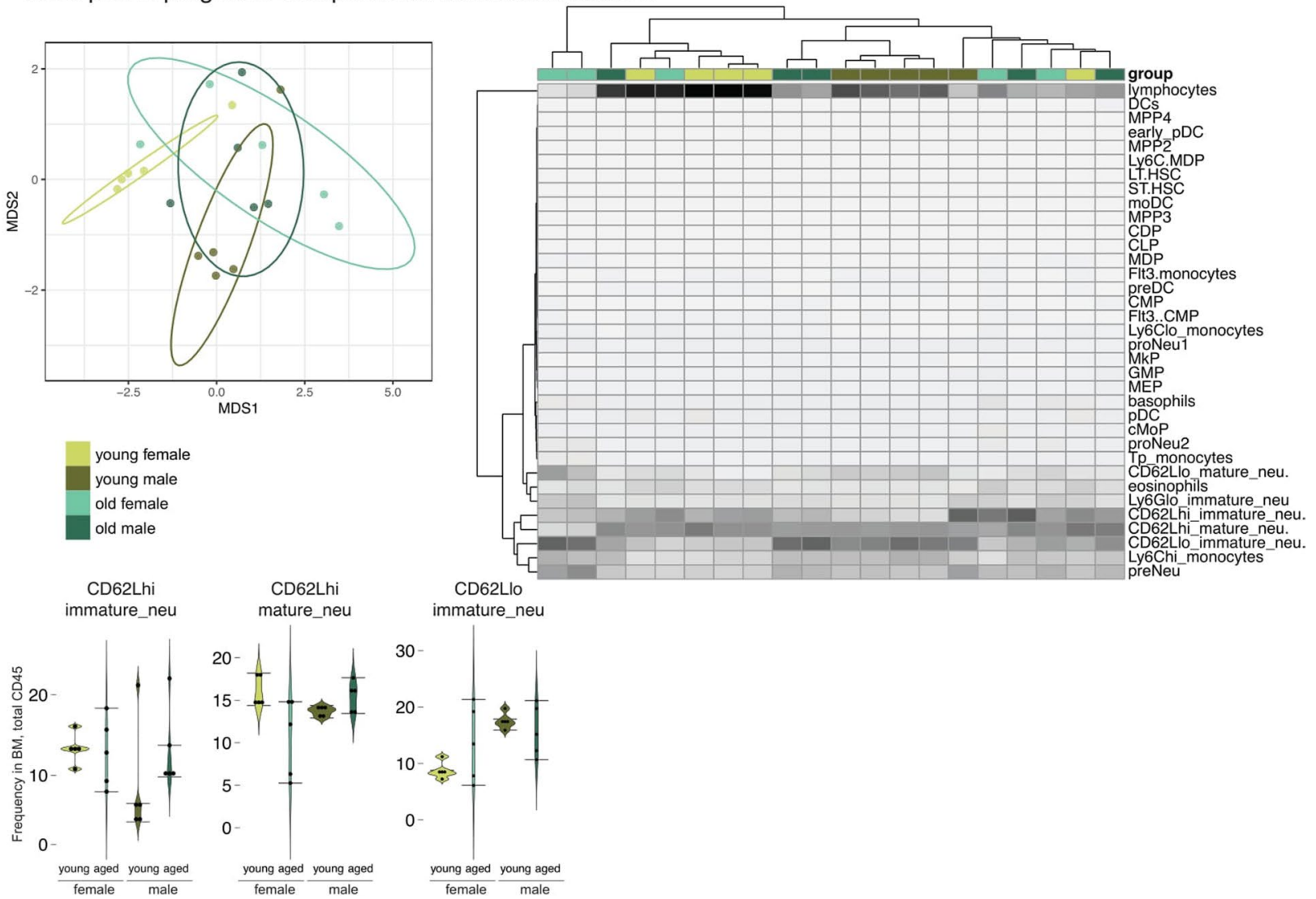

Extended Data Fig. 2 | See next page for caption. 
Extended Data Fig. 2 | MDS analysis reveals sex dimorphism within the progenitor compartment. a: MDS plot showing the lymphoid compartment across sdLNs. The experimental groups (age, sex) are indicated by individual colors (left panel). Heatmap of the mean frequency of all lymphoid populations, calculated within the total CD45 compartment (right panel). b: MDS plot showing the myeloid compartment across sdLNs. The experimental groups (age, sex) are indicated by individual colors (left panel). Heatmap of the mean frequency of all myeloid populations, calculated within the total CD45 compartment (right panel). c: A: MDS plot showing the progenitor compartment across BM. The experimental groups (age, sex) are indicated by individual colors (left panel). Heatmap of the mean frequency of all BM populations, calculated within the total CD45 compartment (right panel). Violin plots depicting frequencies of sex-dimorphic populations in the BM (bottom panel). 
A Expression profile of lymphoid subsets in lung

Young adult

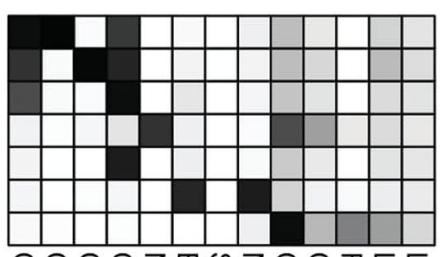

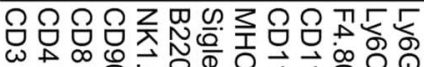

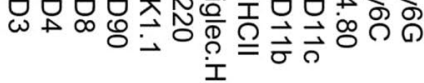

Aged

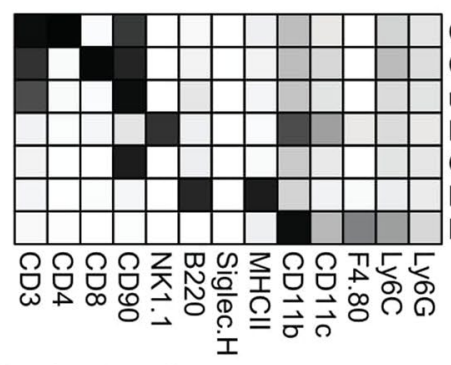

CD4 T cells

CD8 T cells unconv T cells NK cells Other ILCs $B$ cells Myeloid cells

B Expression profile of myeloid subsets in lung Young adult

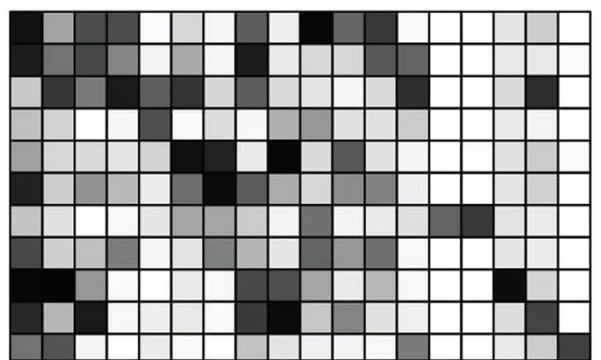

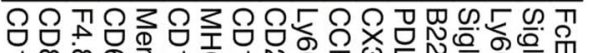

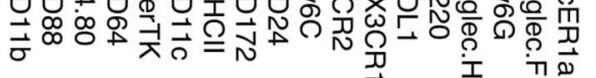

Aged

бำดว

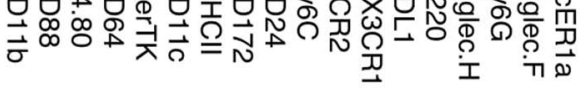

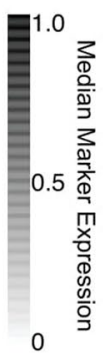

C Frequencies comparing leukocytes in male and female mice

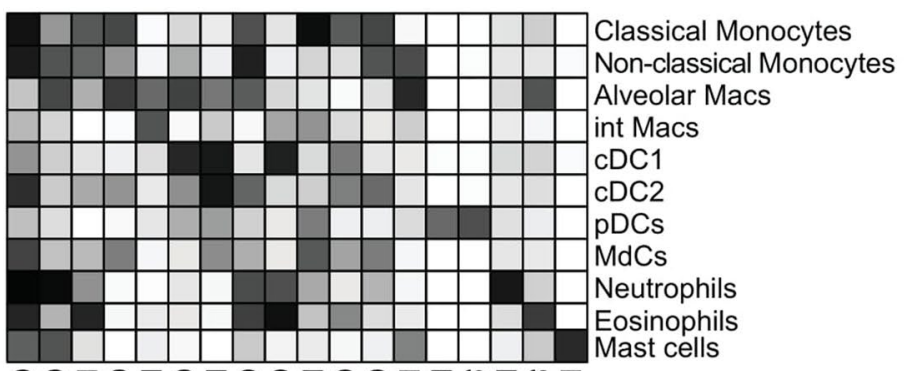

\section{Males}
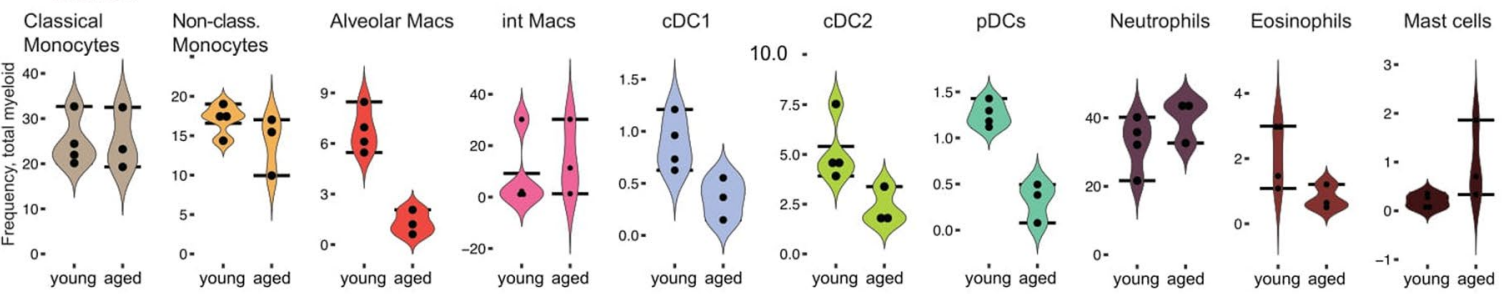

Females
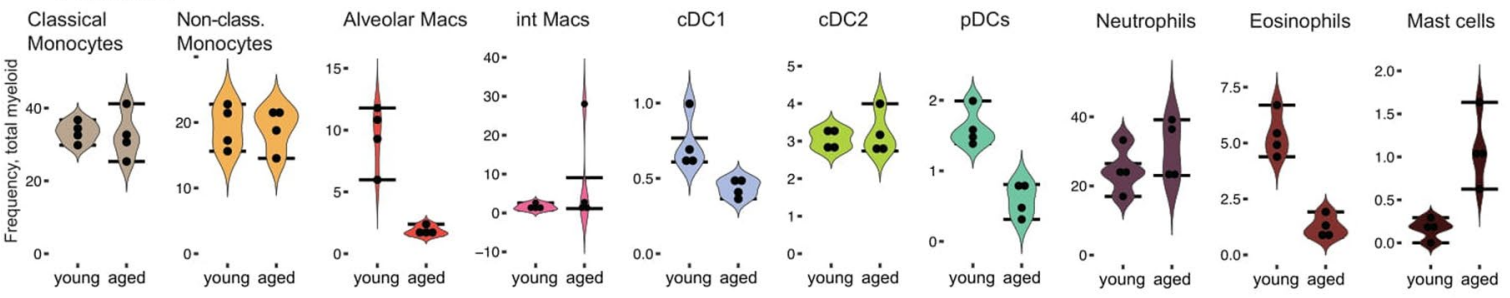

D Discrimination of further lymphoid subsets in validation cohort

Young adult

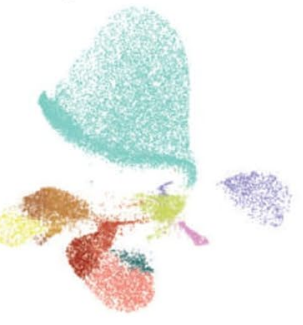

Aged

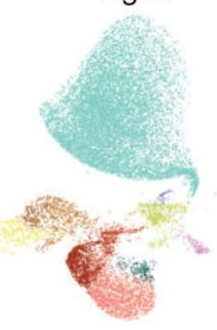

Clusters

CD4 naive T cells

Reg $T$ cells

CD4 TCM cells

CD4 TEM cells

CD4 TEMRA cells

CD8 naive T cells

CD8 TCM cells

CD8 TEM cells

CD8 TEMRA cells

NKT cells

gdT cells

NK cells

Other ILCs

B cells

Myeloid cells

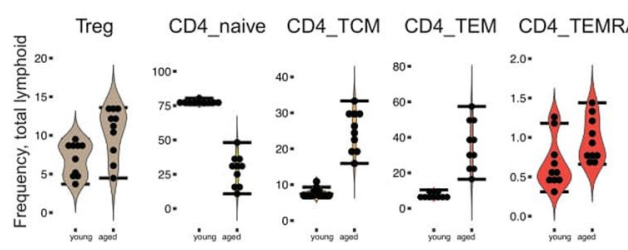

음 CD8_naive 75 CD8_TCM CD8_TEM CD8_TEMRA

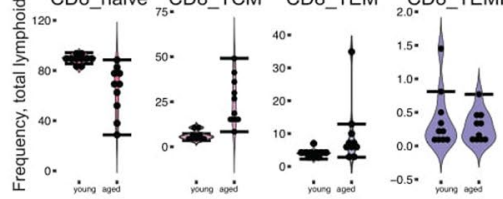

Extended Data Fig. 3 | See next page for caption. 
Extended Data Fig. 3 | Marker expression profile of leukocyte subsets in murine lung, related to Fig. 2. a: Heatmap depicting median marker expression in FlowSOM-derived lymphoid subsets of young adult and aged lung. b: Heatmap depicting median marker expression in FlowSOM-derived myeloid subsets of young adult and aged lung. c: Violin plots depicting frequencies of FlowSOM-generated immune cell clusters in ageing, comparing male and female mice. D: UMAP visualization with FlowSOM overlay depicting the total lymphoid fraction, further characterized in the validation cohort (left panel). Violin plots depicting frequencies of specialized $\mathrm{T}$ cell clusters in ageing. 
A Expression profile of lymphoid and myeloid subsets in sdLN
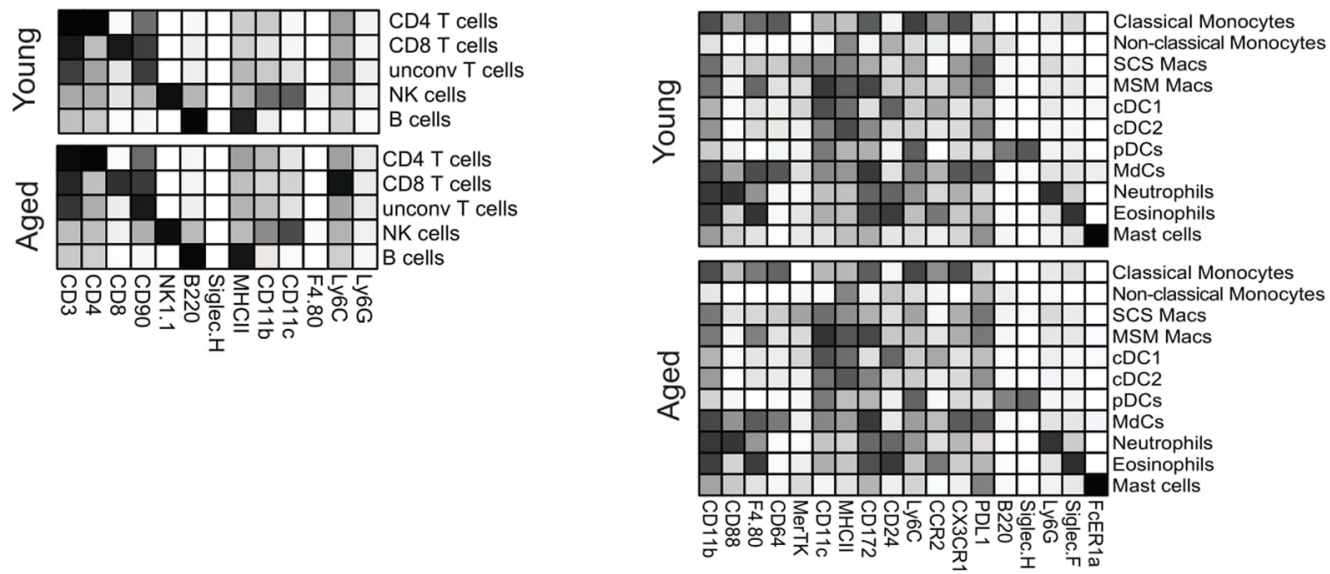

B Frequencies comparing leukocytes in male and female sdLN

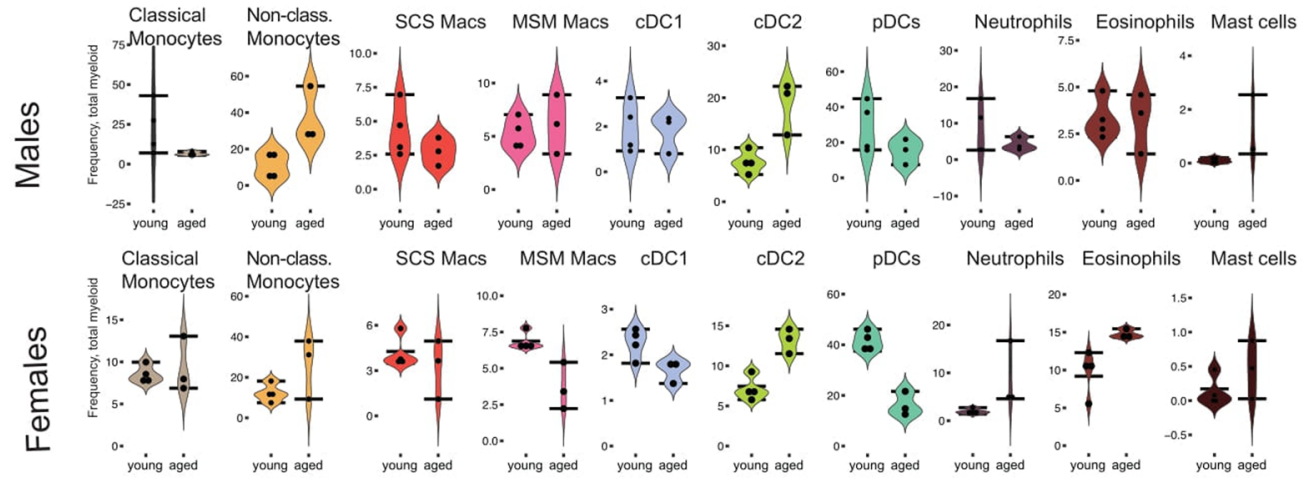

C Expression profile of lymphoid and myeloid subsets in VAT
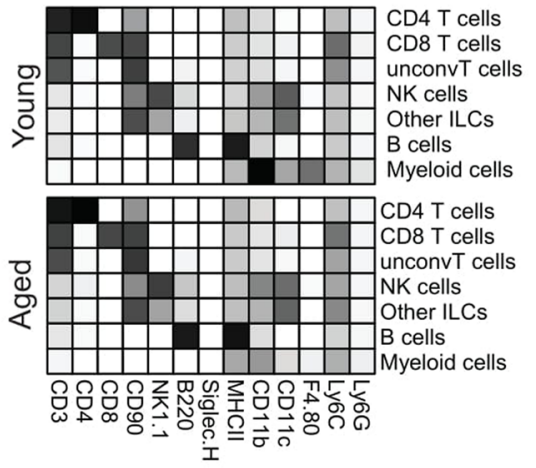

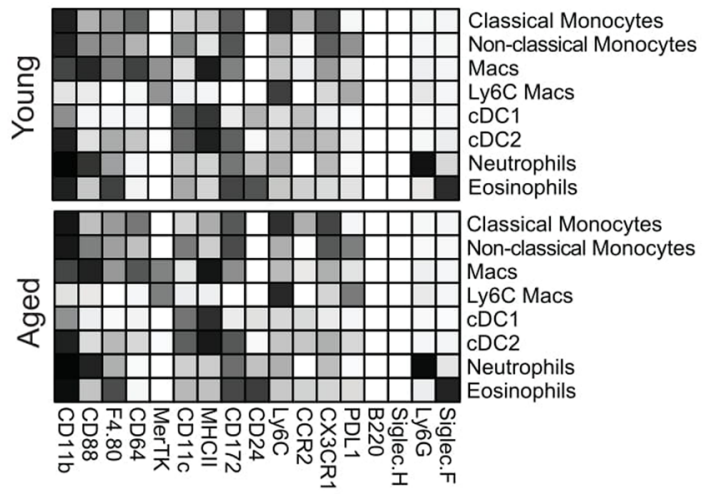

D Frequencies comparing leukocytes in male and female VAT

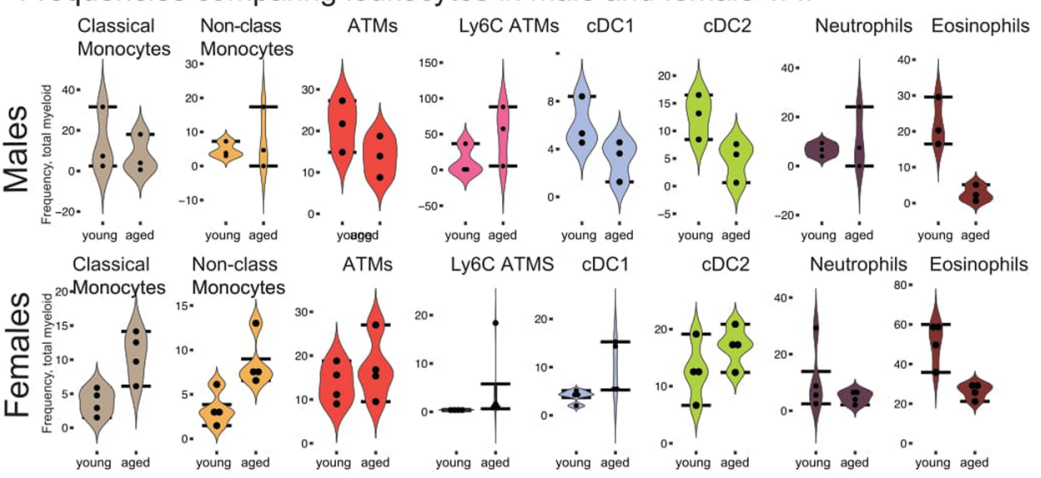

Extended Data Fig. 4 | See next page for caption. 
Extended Data Fig. 4 | Marker expression profile of leukocyte subsets in murine sdLN and VAT, related to Fig. 3. a: Heatmap depicting median marker expression in FlowSOM-derived lymphoid subsets of young adult and aged sdLN (left panel). Heatmap depicting median marker expression in FlowSOMderived myeloid subsets of young adult and aged sdLN (right panel). b: Violin plots depicting frequencies of FlowSOM-generated immune cell clusters in ageing, comparing male and female mice. c: Heatmap depicting median marker expression in FlowSOM-derived lymphoid subsets of young adult and aged VAT (left panel). Heatmap depicting median marker expression in FlowSOM-derived myeloid subsets of young adult and aged VAT (right panel). d: Violin plots depicting frequencies of FlowSOM-generated immune cell clusters in ageing, comparing male and female mice. 
A Representative frequencies of further organs

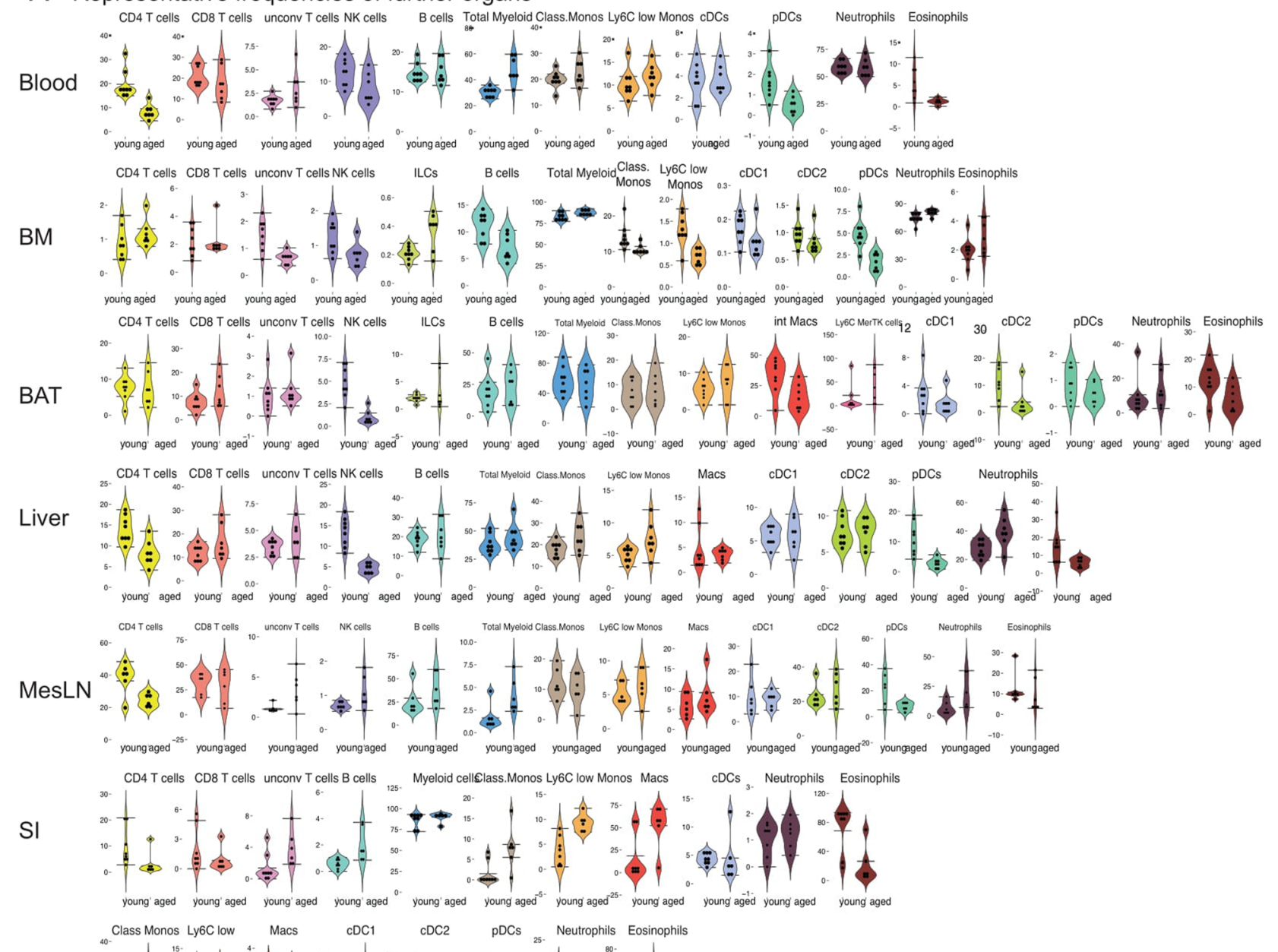

Spleen ${ }^{20}$

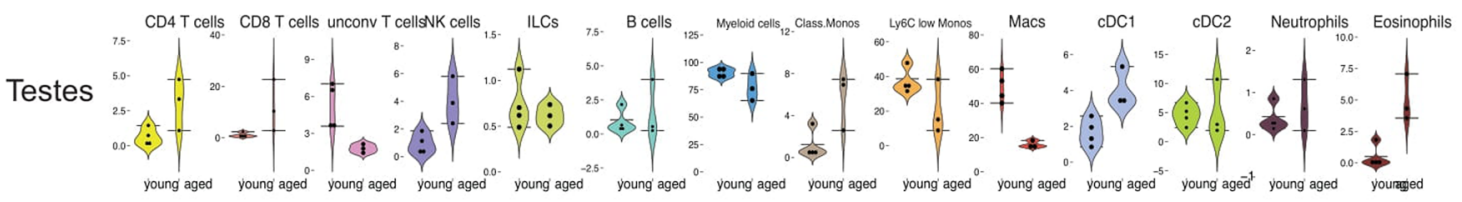

ThymuS

B Volcano plots depicting log fold change of leukocyte populations CD4 T cells NK cells $\mathrm{pDCs}$
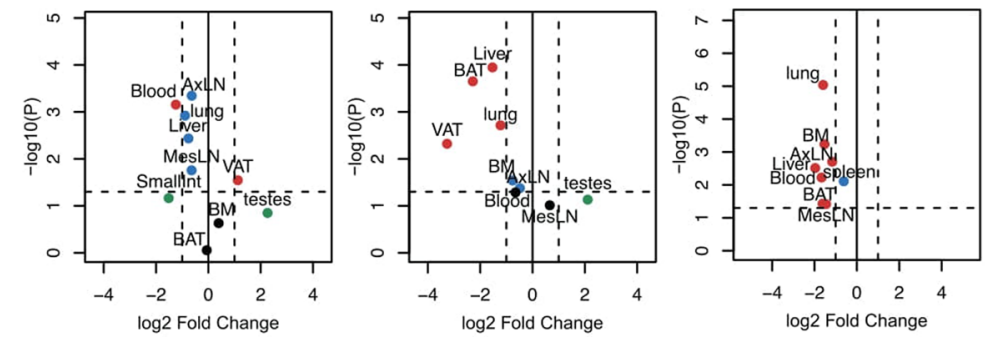

Extended Data Fig. 5 | See next page for caption. 
Extended Data Fig. 5 | Conserved trends in leukocyte abundance in aged versus young mice, related to Figs. 4-5. a: Violin plots depicting frequencies of selective FlowSOM-generated immune cell clusters in ageing, across all tissues included in the immune atlas. b: Volcano plots comparing Log10 (fold change) of aged vs young adult leukocyte populations. Dotted horizontal line depicts cut-off for significant change $(p<0.05)$. Lymphoid populations are calculated as a fraction of total CD45 cells, and myeloid subsets are calculated as a fraction amongst total myeloid cells. Statistical analysis was carried out using non-paired student's $t$ test. $P$ values of less than 0.05 were considered significant and are indicated by the numerical value on the respective graphs. 
A Lymphoid panel lineage gating

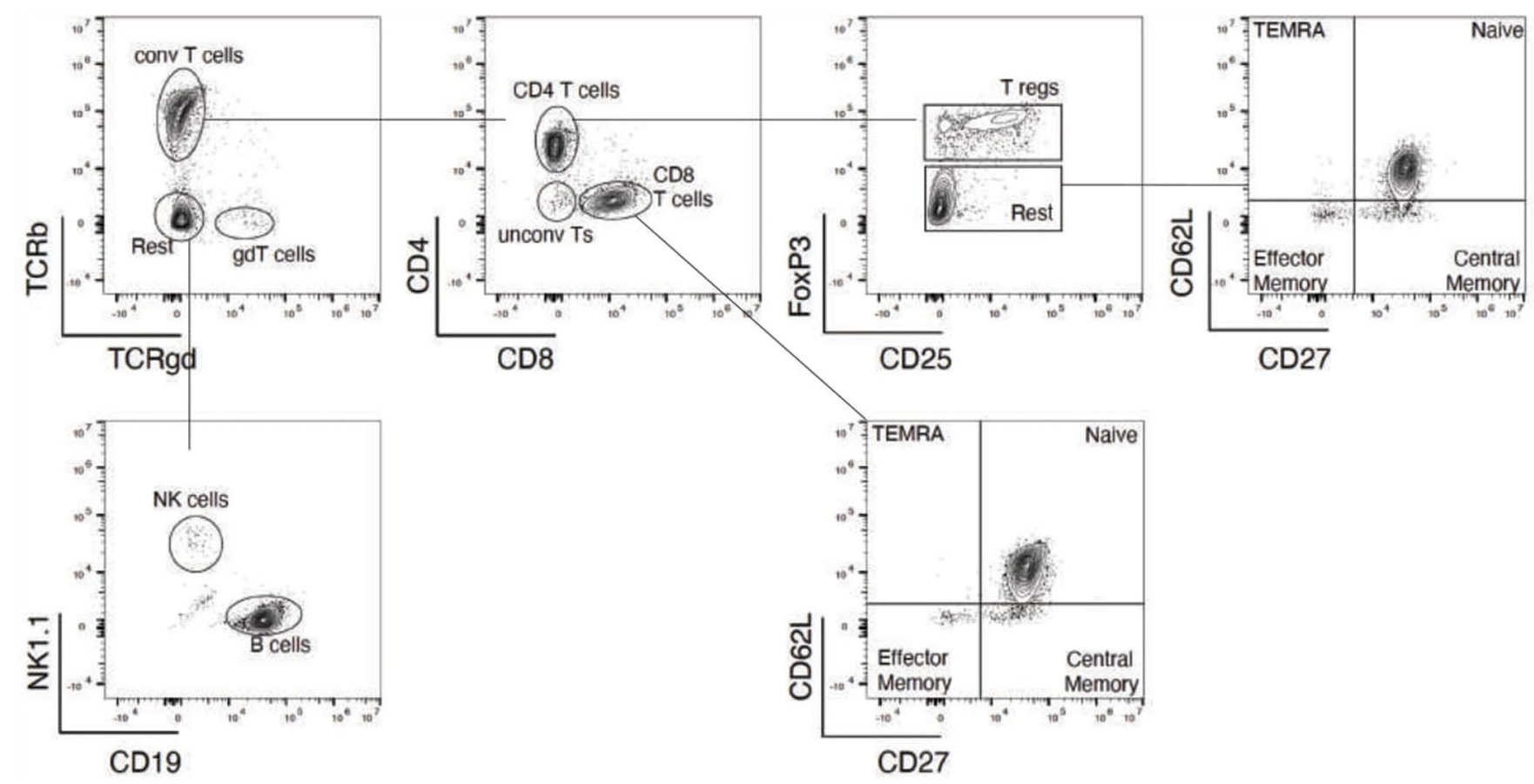

\section{B CD103 expression in CD8 T cells}
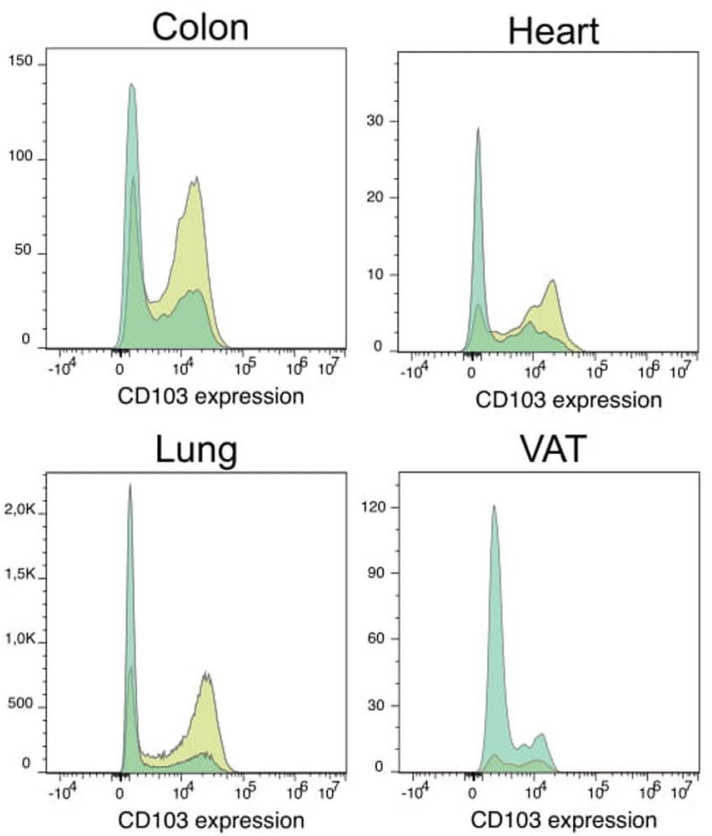

Extended Data Fig. 6 | CD103 expression is downregulated within the naïve CD8 T cell compartment of all aged mouse tissues, related to Fig. 6. a: gating strategy of the lymphoid compartment of the spectral flow-cytometry Validation Cohorts. b: Histograms showing CD103 expression within the naïve CD8 T cells of 4 tissues. Representative young and aged mouse. 
A Heatmap of all identified populations in BM

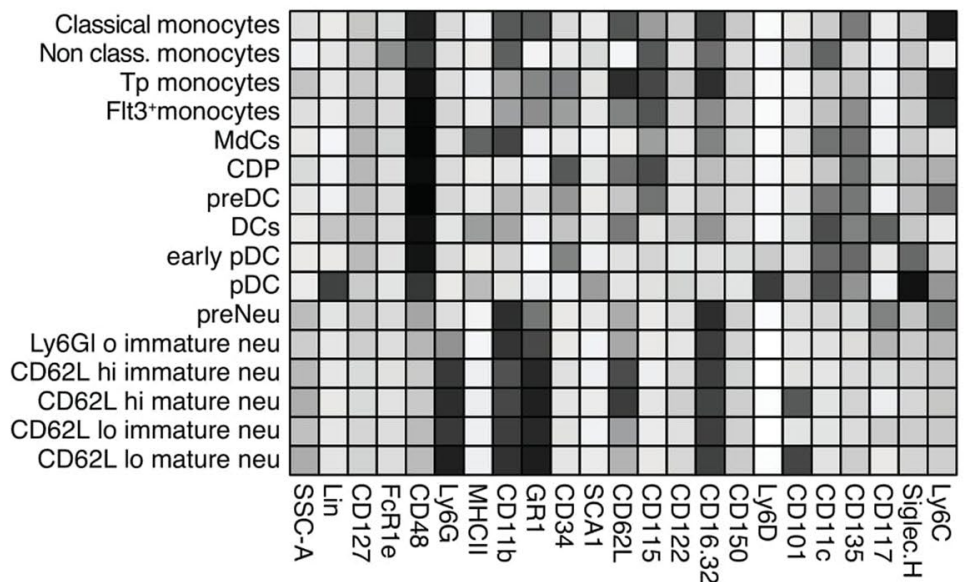

B UMAP of BM Populations

Young adult

Aged

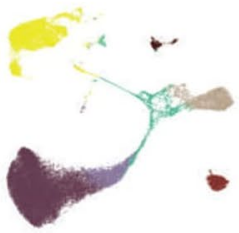

D Frequencies of populations in BM

C Median expression of BM populations

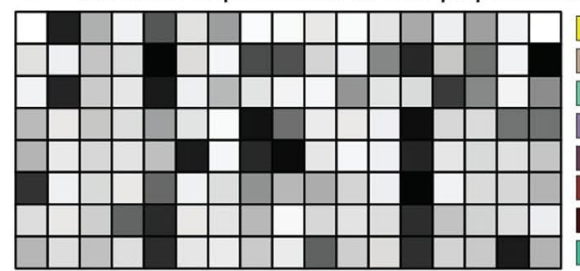

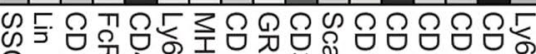

$\square$ Lymphoid cells
$\square$ Mono. phags.
$\square$ pDCs
$\square$ preNeu
Neutrophils
Eosinophils
Basophils
$\square$ Progenitors

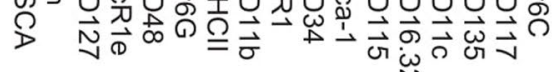

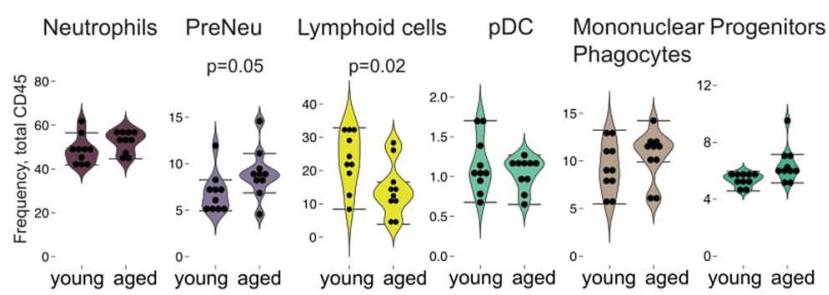

$\mathrm{E}$ Frequencies of $\mathrm{BM}$ populations, total $\mathrm{CD} 45$

\section{Male}

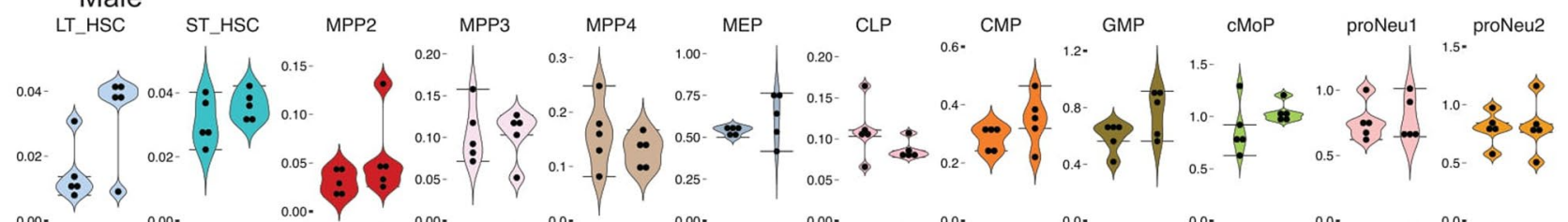

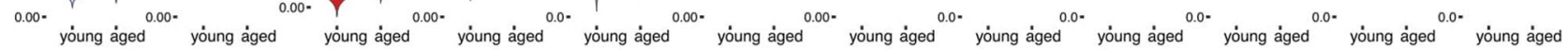

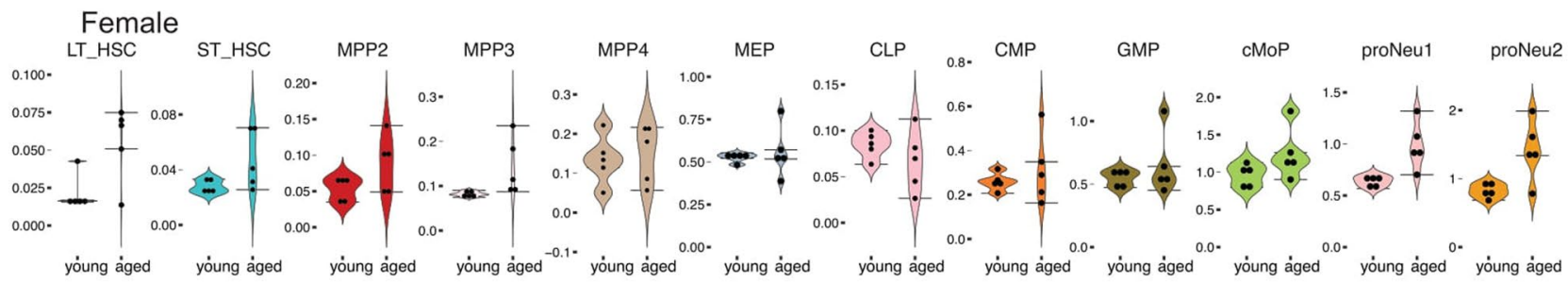

F Manual gating strategy of lymphoid precursors

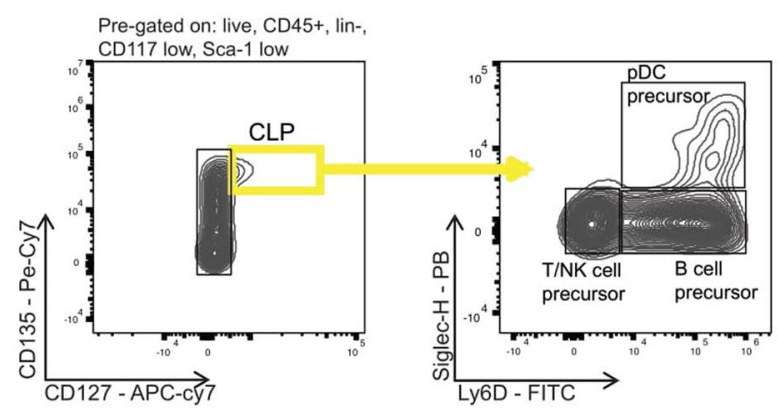

Extended Data Fig. 7 | See next page for caption. 
Extended Data Fig. 7 | Marker expression profile of main hematopoietic cell subsets in murine bone marrow and quantification of committed-CLP subsets in aged bone marrow, related to Fig. 6. a: Heatmap depicting median marker expression in FlowSOM-derived total CD45 subsets in young and aged bone marrow. b: UMAP with FlowSOM overlay showing total CD45pos compartment of young adult and aged BM. c: Heatmap depicting median marker expression in FlowSOM-derived cluster. d: Violin plots depicting frequencies of immune cells among total CD45pos cells of FlowSOM- generated immune cell clusters. Statistical analysis was carried out using non-parametric Mann-Whitney-Wilcoxon tests. The p value was then adjusted for multiple comparisons using a Benjamini-Hochberg test. P values of less than 0.05 were considered significant and are indicated by the numerical value on the respective graphs. e: Violin plots depicting frequencies among total CD45pos cells of FlowSOM- generated progenitor cell clusters. f: Manual gating strategy for CLPs and downstream precursor subsets of the bone marrow, subdivided on the basis of Siglec-H and Ly6D expression. 


\section{Reporting Summary}

Nature Research wishes to improve the reproducibility of the work that we publish. This form provides structure for consistency and transparency in reporting. For further information on Nature Research policies, see our Editorial Policies and the Editorial Policy Checklist.

\section{Statistics}

For all statistical analyses, confirm that the following items are present in the figure legend, table legend, main text, or Methods section.

$\mathrm{n} / \mathrm{a}$ Confirmed

$\bigotimes$ The exact sample size $(n)$ for each experimental group/condition, given as a discrete number and unit of measurement

\ A statement on whether measurements were taken from distinct samples or whether the same sample was measured repeatedly

The statistical test(s) used AND whether they are one- or two-sided

Only common tests should be described solely by name; describe more complex techniques in the Methods section.

Х A description of all covariates tested

$\square$ A description of any assumptions or corrections, such as tests of normality and adjustment for multiple comparisons

A full description of the statistical parameters including central tendency (e.g. means) or other basic estimates (e.g. regression coefficient) AND variation (e.g. standard deviation) or associated estimates of uncertainty (e.g. confidence intervals)

For null hypothesis testing, the test statistic (e.g. $F, t, r$ ) with confidence intervals, effect sizes, degrees of freedom and $P$ value noted Give $P$ values as exact values whenever suitable.

Х $\square$ For Bayesian analysis, information on the choice of priors and Markov chain Monte Carlo settings

$\square$ \ $\square$ or hierarchical and complex designs, identification of the appropriate level for tests and full reporting of outcomes

$\square$ Estimates of effect sizes (e.g. Cohen's $d$, Pearson's $r$ ), indicating how they were calculated

\section{Our web collection on statistics for biologists contains articles on many of the points above.}

\section{Software and code}

Policy information about availability of computer code

Data collection Mass cytometry data was acquired using CyTOF Software version 6.7. For flow cytometry data, SpectroFlo ${ }^{\circledR}$ Software (version 2.0) was used for QC, sample acquisition and spectral unmixing.

Data analysis For mass cytometry data, Flowjo software v10.7.1 and v10.6.2 (TreeStar) was used to identify live cells by using manual gating on event length, DNA (191/r and 193Ir), and live cell marker (195Pt). Cells were further debarcoded using boolean gating. For flow cytometry data, the compensation matrix was corrected in FlowJo (TreeStar) by pre-gating on live immune cells, and the total fraction of live, singlet immune cells was exported. Data were then transformed with an inverse hyperbolic sine (arcsinh) function (mass cytometry: cofactor of 5; spectral flow cytometry: cofactor of 4000) then imported into the R environment (version 3.6.1) for subsequent analysis. UMAPs were generated using the package umap version 0.2.7.0. Frequency plots were generated using the ggplot2 package version 3.3.5, and heatmaps were generated using the pheatmap package version 1.0.12. Force-directed layouts were generated by sampling 1500 cells from each mouse sample, and using the ForceAtlas2 algorithm integrated in the VorteX graphical clustering environment creating unweighted edges between the nodes based on the 10 nearest neighbors. Force directed layout graphs were further modified using the Gephi Toolkit 0.9.2.Toolkit 0.9.2.

Scaffold networks were computed using the improved version of the initial Scaffold package consisting of grappolo, vite and panorama. For the construction of lymphocyte and myeloid cell networks, resulting graphs were rearranged in the igraph framework using the graphopt algorithm (as described in http://www.schmuhl.org/graphopt/). CellCNN-based analysis was performed by training a randomly selected subset of cells. Default values were used for all CellCNN hyperparameters. Default values were used for most CellCNN hyperparameters, except for the following: ncell=3,000; --no_arcsinh; --no_scale; --filter_response_thres 0.3; --train_perc 0.5. MDS plots were generated using the stats package version 4.2 .0 and displayed using ggscatter version 0.4.0. Correlation matrices were calculated using Hmisc version 4.5-0 and generated using circlize version 0.4 .13 in conjunction with corrplot version 0.90. All other plots were generated using ggplot2. R script used for analysis is available through the following repository: 
Policy information about availability of data

All manuscripts must include a data availability statement. This statement should provide the following information, where applicable:

- Accession codes, unique identifiers, or web links for publicly available datasets

- A list of figures that have associated raw data

- A description of any restrictions on data availability

FCS files of the mass and spectral flow-cytometry data, as well as extensive datasets relating to cell counts and statistics are available for download, through the following Repository links - https://zenodo.org/deposit/5593273

\section{Field-specific reporting}

Please select the one below that is the best fit for your research. If you are not sure, read the appropriate sections before making your selection.

\ Life sciences

Behavioural \& social sciences

Ecological, evolutionary \& environmental sciences

For a reference copy of the document with all sections, see nature.com/documents/nr-reporting-summary-flat.pdf

\section{Life sciences study design}

All studies must disclose on these points even when the disclosure is negative.

\begin{tabular}{|c|c|}
\hline Sample size & $\begin{array}{l}\text { Sample sizes were not determined through pre-statistical analyses. However, through post-hoc statistical analyses, we have confirmed that al } \\
\text { statistically meaningful findings (where p values were less than } 0.5 \text { ) were adequately powered ( } 0.8 \text { or higher). }\end{array}$ \\
\hline Data exclusions & $\begin{array}{l}\text { During the acquisition of the mass cytometry cohort, a mouse died shortly prior to the experimental procedures. Hence, the group size of the } \\
\text { old, male cohort was reduced from } n=4 \text { to } n=3 \text {. }\end{array}$ \\
\hline Replication & $\begin{array}{l}\text { For our initial analysis cohort, we devised four experimental groups: } n=4 \text { young adult male, } n=4 \text { young adult female; } n=4 \text { aged female; and } \\
n=3 \text { aged male } C 57 / B L 6 \text { mice. } \\
\text { For our validation cohort } 1 \text {, we devised the following experimental group: } n=5 \text { young adult male, } n=5 \text { young adult female; } n=5 \text { aged female; } \\
\text { and } n=5 \text { aged male C57/BL6 mice. } \\
\text { For our validation cohort } 2 \text {, we procured: } n=5 \text { juvenile male, } n=5 \text { young adult male, } n=5 \text { juvenile female, } n=5 \text { young adult female; } n=5 \text { aged } \\
\text { female; and } n=5 \text { aged male C57/BL } 6 \text { mice. }\end{array}$ \\
\hline
\end{tabular}

Randomization Since animals were allocated to various experimental groups due to their age or sex, randomisation was deemed not possible for this experimental setup.

Blinding Due to the nature of our experiments (comparing the immune system of young adult vs aged mice), it was not possible to introduce blinding to the experimental protocol since the experimenters could visibly see the difference between the animals. However, data analysis was carried out using machine learning algorithms where samples were labeled numerically.

\section{Reporting for specific materials, systems and methods}

We require information from authors about some types of materials, experimental systems and methods used in many studies. Here, indicate whether each material, system or method listed is relevant to your study. If you are not sure if a list item applies to your research, read the appropriate section before selecting a response.

\begin{tabular}{l|l} 
Materials \& experimental systems \\
\hline $\mathrm{n} / \mathrm{a}$ & Involved in the study \\
\hline & $\bigotimes$ Antibodies \\
\hline & $\square$ Eukaryotic cell lines \\
$\square$ & $\square$ Palaeontology and archaeology \\
$\square$ & $\square$ Animals and other organisms \\
$\square$ & $\square$ Clinical data \\
$\square$ & $\square$ Dual use research of concern
\end{tabular}

\begin{tabular}{l|l} 
Methods \\
\hline $\mathrm{n} / \mathrm{a}$ & Involved in the study \\
$\square$ & $\square$ ChIP-seq \\
$\square$ & $\square$ Flow cytometry \\
$\square$ & $\square$ MRI-based neuroimaging
\end{tabular}

\section{Antibodies}

Antibodies used

All antibodies used in our studies have been described in detail within Supplementary Tables 2-5. 

RRID: AB_312967; anti-mouse CD45 clone 30-F11, 105-Pd BioLegend Cat \# 103102; RRID: AB_312967; anti-mouse CD45 clone $30-$ F11, 106-Pd BioLegend Cat \# 103102; RRID: AB_312967; anti-mouse CD45 clone 30-F11, 108-Pd BioLegend Cat \# 103102; RRID: AB_312967; anti-mouse CD45 clone 30-F11, 110-Pd BioLegend Cat \# 103102; RRID: AB_312967; anti-mouse CD45 clone 30-F11, 115-Pd BioLegend Cat \# 103102; RRID: AB_312967; anti-mouse Ly6G clone 1A8, 141-Pr Fluidigm Cat \# 3141008B; anti-mouse CD11b clone M1/70, 148-Nd Fluidigm Cat \# 3148003B; anti-mouse Ly6C clone HK1.4, 150-Nd Fluidigm Cat \# 3150010B; anti-mouse CD64 clone X54-5/7.1, 151-Eu Fluidigm Cat \# 3151012B; anti-mouse CD3 clone 145-2C11, 152-Sm Fluidigm Cat \# 3152004B; antimouse Ter119 clone TER-119, 154-Sm Fluidigm Cat \# 3154005B; anti-mouse CD14 clone Sa14-2, 156-Gd Fluidigm Cat \# 3156009B; anti-mouse CD117 clone 2B8, 166-Er Fluidigm Cat \# 3166004B; anti-mouse Sca-1 clone D7, 169-Tm Fluidigm Cat \# 3169015B; antimouse CX3CR1 clone SA011F11 BioLegend Cat\# 149002; RRID: AB_2564313; anti-mouse CD88 clone 20/70 BioLegend Cat\# 135802 RRID: AB_1953295; anti-mouse Siglec-F clone E50-2440 BD Biosciences Cat\# 552125; RRID: AB_394340; anti-mouse CD11c clone N418 BioLegend Cat\# 117302; RRID: AB_313771; anti-mouse B220 clone RA3.3A1 Bio X Cell Cat\# BE0067; RRID: AB_1107651; antimouse PDL1 clone 10F.9G2 Bio X Cell Cat\# BE0101; RRID: AB_10949073; anti-mouse CD90 clone T24/31 Bio X Cell Cat\# BE0212; RRID: AB_2687698; anti-mouse Siglec-H clone 551 BioLegend Cat\# 129602; RRID: AB_1227757; anti-mouse Siglec-1 clone 3D6.112 BioLegend Cat\# 142402; RRID: AB_10916523; anti-mouse MerTK polyclonal, biotin R\&D Systems Cat\# BAF591; RRID: AB_2098563; anti-mouse CD135 clone A2F10 eBioscience Cat\# 14-1351-82; RRID: AB_467481; anti-mouse CD24 clone J11d Bio X Cell Cat\# BE0227; RRID: AB_2687710; anti-mouse CCR2 clone 475301, APC R\&D Systems Cat\# FAB5538A; RRID: AB_10645617; anti-mouse F4/80 clone Cl:A3-1 BioRad Cat\# MCA497G; RRID: AB_872005; anti-mouse CD38 clone 90 BioLegend Cat\# 102702; RRID: AB_312923; anti-mouse CD16/32 clone 93 BioLegend Cat\# 101302; RRID: AB_312801; anti-mouse CD206 clone CO8C2 BioLegend Cat\# 14170210900233; anti-mouse NK1.1 clone PK136 BioLegend Cat\# 108702; RRID: AB_313389; anti-mouse CD86 clone GL-1 Bio X Cell Cat\# BE0025; RRID: AB_1107678; anti-mouse CD172 clone P84 BioLegend Cat\# 144002; RRID: AB_11203711; anti-mouse FCER1a clone MAR-1 BioLegend Cat\# 134302; RRID: AB_1626078; anti-APC clone APC003 BioLegend Cat\# 409002; RRID: AB_345357; anti-biotin clone 1D4-C5 BioLegend Cat\# 408002; RRID: AB_10642032

For spectral flow cytometry analysis of 11 organs (see method section in the main text) we used the following antibodies: BUV805 CD8 clone 53-6.7 BD 564920; BUV737 CD44 clone IM7 BD 612799; BUV661 CD11b clone M1/70 BD 565080; BUV496 CD4 clone GK1.5 BD 564667; BV785 PD1 clone 29F.1A12 BioLegend 135225; BV711 NK1.1 clone PK136 BioLegend 108745; BV650 CD25 clone PC61 BioLegend 102038; BV605 CD69 clone H1.2F3 BioLegend 104530; BV570 CD62L clone MEL-14 BioLegend 104433; BV510 KLRG1 clone 2F1/KLRG1 BioLegend 138421; BV421 CD103 clone 2E7 BioLegend 121422; PerCP-eFluor710 TCRgd clone GL3 eBioscience 46-5711-82; BB700 CD115 clone AfS98 Biolegend 135508; BB700 CD14 clone Sa14-2 BioLegend 123305; BB700 F480 clone CI:A3-1 AbD Serotec MCA497B(B); BB700 Ly6g clone 1A8 1A8 BioLegend; BB700 Ter119 clone Ter-119 BioLegend 116204; FITC NKp46 clone 29AI.4 eBioscience 11-3351-82; PE-Cy7 CD27 clone LG.3A10 BioLegend 124216

PE-Cy5.5 CD19 clone ebio 1D3 eBioscience 35-0193-82; PE-Cy5 TCRb clone H57-597 BioLegend 109209; PE CD49b clone DX5 BioLegend 108908; APC Fire 750 CD73 clone TY/11.8 BioLegend 127232; BB630 Streptavidin BD costum; BV480 KI67 clone B56 BD 566109; PE-eF610 FOXP3 clone FJK-16s eBioscience 61-5773-82; AF647 GZMB clone GB11 BioLegend 515406; BUV805 CD4 clone GK1.5 BD 612900; BUV737 CD11b clone M1/70 BD 564443; BUV563 Ly6G clone 1A8 BD 565707; BUV496 CD24 clone M1/69 BD 564664; Live/Dead Blue live/dead fix blue ; BUV395 CD45 clone 30-F11 BD 564279; SB780 MerTK clone DS5MMER eBioscience 78-5751-82; BV750 CD88 clone 20/70 BD 747227; BV711 Ly6C clone HK1.4 BioLegend 128037; BV650 XCR1 clone ZET BioLegend 148220; BV605 CD274 clone 1OF.9G2 BioLegend 124321; BV570 CD44 clone IM7 BioLegend 103037; BV510 CD16/32 clone 93 Biolegend 101333; efluor450 LYVE1 clone ALY7 eBioscience 48-0443-82; BV421 B220 clone RA3-6B2 BD 558108; PerCP-eFluor710 Siglec H clone 551 eBioscience 46-0333-80; BB700 MHCll clone M5/114.15.2 BD 746197; AF 532 CD64 Biotin clone X54-5/7.1 BioLegend 139318; AF488 FcER1a clone MAR1 Biolegend 134329; BB515 Siglec F clone E50-2440 BD 564514; PE-Cy7 Sirpa clone P84 BioLegend 144007; PE-Cy5.5 CD11c clone N418 eBioscience 35-0114-82; PE-Cy5 F4/80 clone BM8 BioLegend 123112; PE-eFluor610 CX3CR1 clone SA011F11 BioLegend 149013; PE ESAM clone 1G8/ESAM Biolegend 136203; PE Siglec-1 clone SER-4 eBioscience 12-5755-80; APC-Cy7 EPCAM clone G8.8 Biolegend 118218; AF700 CD206 clone C068C2 BioLegend 141734; Alexa fluor 647 TIM4 clone RMT4-54 BioLegend 130007; APC CD3 clone 17A2 BioLegend 100236; APC CD19 clone eBio1D3 invitrogen 17-0193-82; APC NK1.1 clone PK136 BD 550627, APC Ter119 clone Ter-119 BioLegend 17-5921-81

For spectral flow cytometry analysis of the bone marrow, we used the following antibodies:

BUV395 anti-mouse CD45 (30F11, BD Biosciences, dilution 1:400), BUV737 anti-mouse CD11b (N418, BD Biosciences, dilution 1:400), PE-Cy5.5 anti-mouse CD11C (N418, Biolegend, dilution 1:400), APC anti-mouse CD3 (17A2, Biolegend, dilution 1:200), BUV546 antimouse Ly6G (1A8, Biolegend, dilution 1:200), FITC anti-mouse Ly6C (HK1.4, Biolegend, dilution 1:200), APC anti-mouse CD19 (1D3, Biolegend, dilution 1:400), APC anti-mouse Ter119 (Ter-119, Biolegend, 1:200), BV711 anti-mouse CD16/32 (93, Biolegend,1:200), Biotin anti-mouse CD135 (A2F10, Biolegend, dilution 1:100), BV421 anti-mouse CD34 (SA376A4, eBioscience, 1:100), BV605 antimouse CD115 (AFS98, Biolegend, dilution 1:100), BUV661 anti-mouse I-A/I-E (M5/114.15.2, BD Biosciences, dilution 1:400), APC PEefluor610 anti-mouse CD117 (2B8, eBioscience, dilution 1:200) and BV510 anti-mouse SCA-1 (D7, Biolegend, dilution 1:200), BV795 anti-mouse CD150 (TC15-12F12.2, Biolegend, dilution 1:100), AlexaFluor700 anti-mouse CD48 ( HM48-1, Biolegend, dilution 1:100), APC-Cy7 anti-mouse CD127(A7R34, Biolegend, dilution 1:100), APC anti-mouse B220 (RA3-6B2, Biolegend, dilution 1:200), PE-Cy7conjugated Streptavidin (BD Biosciences, dilution 1:400), BUV801 anti-mouse GR1(RB6-8C5, BD Biosciences dilution 1:200), Pacific blue mouse SiglecH (551, Biolegend, dilution 1:200), Alexafluor488 anti-mouse Ly6D (551, Biolegend, dilution 1:100), BV650 antimouse CD122 (TM-Beta1, dilution 1:200), BV750 anti-mouse SiglecF (E50-2440, BD Bioscences, dilution 1:200), PE anti-mouse CD101 (Moushi101, eBioscience, dilution 1:400), Alexafluor647 anti-mouse FceRla (MAR-1, eBioscience, 1:200), APC anti-mouse NK1.1 (Biolegend, 1:200). associated product web-pages. Panel tests were carried out prior to experimental procedures, to ensure that the stainings looked appropriate prior to acquiring final cohorts. There, the antibodies were combined into the panels described in supplementary tables 2-5 in the described dilutions, and tested on all WT mouse tissues included in our analysis: lung, liver, visceral adipose tissue (VAT), brown adipose tissue (BAT), small intestine, thymus, testes, mesenteric lymph nodes (MesLNs), skin-draining lymph nodes (sdLNs), spleen, blood and bone marrow (BM). 
Policy information about studies involving animals; ARRIVE guidelines recommended for reporting animal research

Laboratory animals

C57BL/6JRj, wild-type mice were acquired from Janvier laboratories, where the animals were housed in the same housing facility from birth until purchase. Then, the animals were acclimatised, together, at the animal housing facility of the University of Zurich for at least 2 weeks before experimental procedures. Male and female mice were aged around 7-8 weeks ("young"), 16 weeks

("middle") or 18-20 months ("aged"). Our mice showed no physical signs of illness or macroscopic signs of cancerous growths at the time of analysis. Both our initial and validation cohorts of mice have been housed under identical conditions from birth until purchase by the vendor. For our initial analysis cohort, we devised four experimental groups: $n=4$ young adult male, $n=4$ young adult female; $n=4$ aged female; and $n=3$ aged male C57/BL6 mice. For our validation cohort 1, we devised the following experimental group: $n=5$ young adult male, $n=5$ young adult female; $n=5$ aged female; and $n=5$ aged male C57/BL6 mice. For our validation cohort 2, we procured: $n=5$ juvenile male, $n=5$ young adult male, $n=5$ juvenile female, $n=5$ young adult female; $n=5$ aged female; and $n=5$ aged male C57/BL6 mice. All animal experiments performed in this study were approved by the Cantonal Veterinary Office Zurich.

Wild animals The study did not involve wild animals.

Field-collected samples The study did not involve samples collected from the field.

Ethics oversight All animal experiments performed in this study were approved by the Cantonal Veterinary Office Zurich.

Note that full information on the approval of the study protocol must also be provided in the manuscript.

\section{Flow Cytometry}

Plots

Confirm that:

\The axis labels state the marker and fluorochrome used (e.g. CD4-FITC).

\The axis scales are clearly visible. Include numbers along axes only for bottom left plot of group (a 'group' is an analysis of identical markers).

\All plots are contour plots with outliers or pseudocolor plots.

\A numerical value for number of cells or percentage (with statistics) is provided.

\section{Methodology}

Sample preparation

Instrument

Software

Cell population abundance

Gating strategy
Tissues from mice (lung, liver, visceral adipose tissue, brown adipose tissue, small intestine, thymus, testes, mesenteric lymph nodes, axillary lymph nodes, spleen, blood and bone marrow) were harvested as described previously 4. Briefly, mice were sacrificed by intra-peritoneal (i.p.) injection of pentobarbital ( $50 \mathrm{uL}$ at $300 \mathrm{mg} / \mathrm{ml}$ ) followed by trans-cardiac perfusion with PBS and heparin $(5 \mathrm{U} / \mathrm{ml})$. Each organ was harvested and cut into small pieces, using scissors, inside an Eppendorf tube. Next, the samples were incubated with digestion medium (HBSS++ with 2\% FCS, $2 \mathrm{mM} \mathrm{HEPES,} \mathrm{DNAse} \mathrm{(50ul/10ml)} \mathrm{and} \mathrm{Collagenase}$ IV) at 37 degreesC whilst shaking.

Below is a list of Collagenase concentrations, volumes used per sample and incubation period for each organ analysed:

LNs $\times 2$, spleen $0.2 \mathrm{mg} / \mathrm{mL}, 3 \mathrm{ml}, 20$ minutes

VAT, BAT $0.2 \mathrm{mg} / \mathrm{mL}, 5 \mathrm{ml}, 30$ minutes

Thymus $0.2 \mathrm{mg} / \mathrm{mL}, 4 \mathrm{ml}, 45$ minutes

Lung $0.4 \mathrm{mg} / \mathrm{mL}, 4 \mathrm{ml}, 30$ minutes

Testes $0.4 \mathrm{mg} / \mathrm{mL}, 4 \mathrm{ml}, 30$ minutes

Liver $1.0 \mathrm{mg} / \mathrm{mL}, 5 \mathrm{ml}, 20$ minutes

Intestine $0.4 \mathrm{mg} / \mathrm{mL}, 5 \mathrm{ml}, 45$ minutes

BM - Flush with PBS and filter

Blood - RCB lysis for 5 minutes on ice ( $2 \mathrm{~mL}$ per $100 \mathrm{ul}$ of blood).

Enzymatic digestion was stopped with EDTA $5 \mathrm{mM}$. The samples were homogenized using a syringe and a $24 \mathrm{G}$ needle, and the homogenate was filtered through a 100uM cell strainer. SLTs were subjected to Red Blood Cell lysis (Biolegend) (5 minutes at room temperature). The CNS cell suspensions were further enriched by Percoll gradient $(30 \%)$ centrifugation $\left(1590 \mathrm{~g}, 4^{\circ} \mathrm{C}, 30\right.$ min, no brake) and thymus samples too (centrifugation at $2750 \mathrm{rpm}, 4^{\circ} \mathrm{C}, 30 \mathrm{~min}$, no brake). Spleen samples from the initial cohort were subjected to myeloid cell enrichment using negative bead selection (MACS MicroBeads and Column, Miltenyi Biotec) for ter119, CD3, CD19 and CD5. The samples were then ready for labelling with antibodies for mass- or fluorescencecytometry.

Mass cytometry (CyTOF) data was acquired using a Fluidigm Helios. Spectral flow cytometry data was acquired using a Cytek Aurora.

Flow cytometry data was collected using SpectroFlo software. The compensation matrix was corrected in FlowJo v10.6.2 (TreeStar) by pre-gating on live immune cells, and the total fraction of live, singlet immune cells was exported for further computational analysis.

Samples were not sorted for this study.

The compensation matrix was corrected in FlowJo (TreeStar). CD45+ live single immune cells (FSC vs SSC, singlet, live CD45- 
$\bigotimes$ Tick this box to confirm that a figure exemplifying the gating strategy is provided in the Supplementary Information. 